\title{
Tip Leakage Losses in a Linear Turbine Cascade
}

by

\section{PETER T. DISHART}

Thesis submitted to the Faculty of the

Virginia Polytechnic Institute and State University

in partial fulfillment of the requirements for the degree of

Master of Science

in

Mechanical Engineering

APPROVED:

Dr. F. J. Pierce

Dr. H. L. Moses

$11 / 12 / 87$

Blacksburg, Virginia 


\title{
Tip Leakage Losses in a Linear Turbine Cascade
}

by

\author{
PETER T. DISHART \\ Dr. J. Moore, Chairman \\ Mechanical Engineering
}

(ABSTRACT)

An investigation of tip leakage flow and its effects on loss production was performed on a large scale linear turbine cascade having a tip gap measuring $2.1 \%$ of the blade height. The Reynolds number based on axial chord and cascade exit velocity was $4.5 \times 10^{5}$. The experimental work began with a visualization study of the flow in and around the tip gap. The actual flow measurements consisted of two phases, the tip gap exit plane measurements for determination of the losses incurred within the tip gap, and the downstream measurements for determination of the overall cascade losses. The downstream measurements made $140 \%$ of an axial chord downstream of the blade leading edges show the development of the leakage flow and its associated losses.

Numerical analyses of the data were used to evaluate various flow properties at both the tip gap exit plane and the downstream measurement plane. Using the measured downstream flow, a mixing analysis was performed to estimate the maximum loss of the cascade.

Models of the flow were developed to explain and quantify the various factors contributing to the cascade's overall loss. At a particular downstream location, the additional loss due to tip leakage was found to be the sum of the measured loss at the tip gap exit plane and the amount of tip gap secondary kinetic energy which had been dissipated by that downstream location. 


\section{Acknowledgements}

I am particularly indebted to Dr. John Moore who conceived this project. His understanding of when to be a supervisor and when to be a coworker made my graduate studies a rewarding and enjoyable experience.

I would also like to thank Dr.'s F. J. Pierce and H. L. Moses for their participation as committee members.

The support and encouragement from my friends and fellow graduate students ( not mutually exclusive) was also of great importance to me.

Additional thanks to Jeffrey Wynne for his fast fingered typing of this thesis. 


\section{Table of Contents}

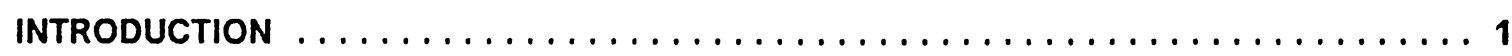

LITERATURE REVIEW $\ldots \ldots \ldots \ldots \ldots \ldots \ldots \ldots \ldots \ldots \ldots \ldots \ldots \ldots$

2.1 LOSS PREDICTION AND REDUCTION $\ldots \ldots \ldots \ldots \ldots \ldots \ldots \ldots \ldots \ldots$

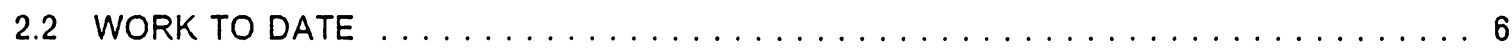

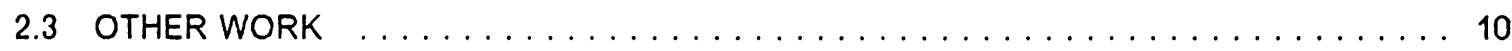

2.4 PRESENT STUDY $\ldots \ldots \ldots \ldots \ldots \ldots \ldots \ldots \ldots \ldots \ldots \ldots \ldots \ldots \ldots \ldots$

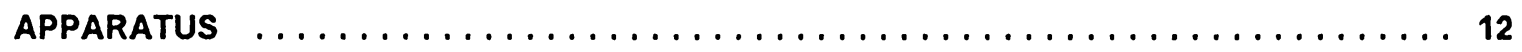

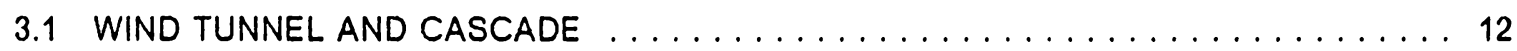

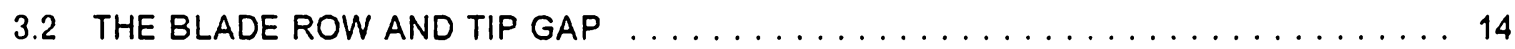

3.3 MEASURING EQUIPMENT $\ldots \ldots \ldots \ldots \ldots \ldots \ldots \ldots \ldots \ldots \ldots$

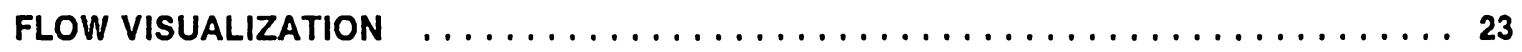

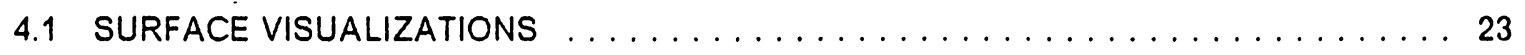

4.1 .1 BOTTOM ENDWALL $\ldots \ldots \ldots \ldots \ldots \ldots \ldots \ldots \ldots \ldots \ldots \ldots \ldots$

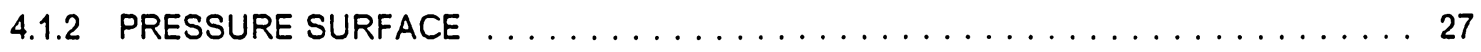




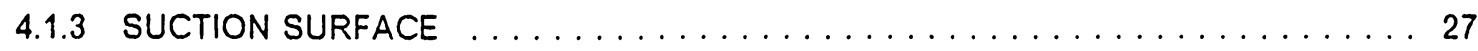

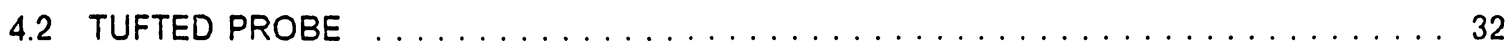

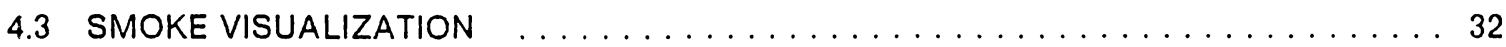

MEASUREMENTS AND PROCEDURE $\ldots \ldots \ldots \ldots \ldots \ldots \ldots \ldots \ldots \ldots$

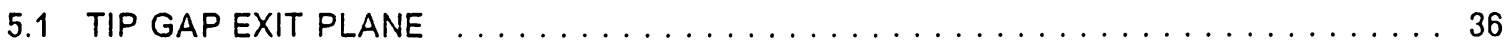

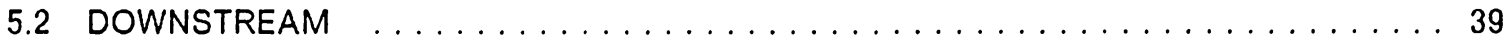

RESULTS, ANALYSIS AND DISCUSSION $\ldots \ldots \ldots \ldots \ldots \ldots \ldots \ldots \ldots \ldots \ldots$

6.1 FLOW CONDITIONS $\ldots \ldots \ldots \ldots \ldots \ldots \ldots \ldots \ldots \ldots \ldots \ldots \ldots \ldots \ldots$

6.2 FLOW MEASUREMENTS AT THE TIP GAP EXIT $\ldots \ldots \ldots \ldots \ldots \ldots \ldots \ldots$

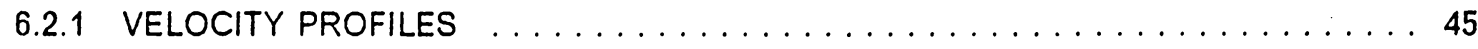

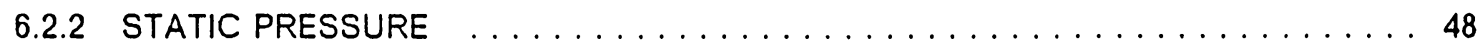

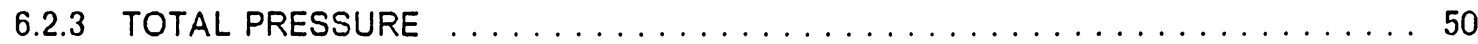

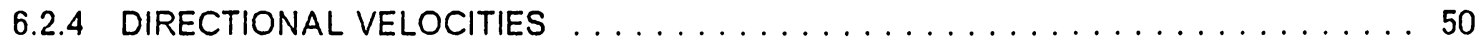

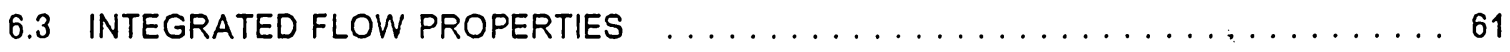

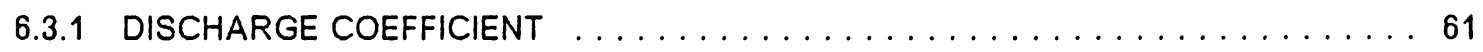

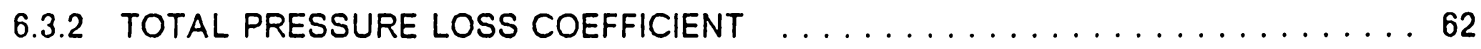

6.3 .3 OVERALL TIP LEAKAGE COEFFICIENTS $\ldots \ldots \ldots \ldots \ldots \ldots \ldots$

6.4 LOSS PRODUCTION UP TO THE TIP GAP EXIT $\ldots \ldots \ldots \ldots \ldots \ldots \ldots \ldots \ldots \ldots$

6.5 DOWNSTREAM MEASUREMENTS $\ldots \ldots \ldots \ldots \ldots \ldots \ldots \ldots \ldots \ldots \ldots$

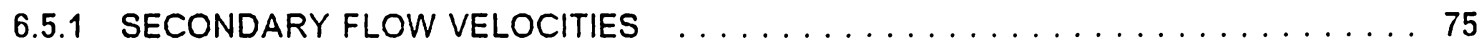

6.5 .2 TOTAL PRESSURE $\ldots \ldots \ldots \ldots \ldots \ldots \ldots \ldots \ldots$

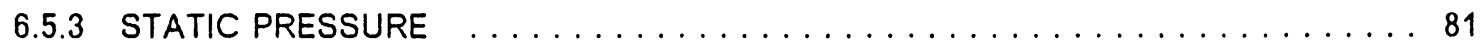

6.5 .4 AVERAGED FLOW PROPERTIES $\ldots \ldots \ldots \ldots \ldots \ldots \ldots \ldots$

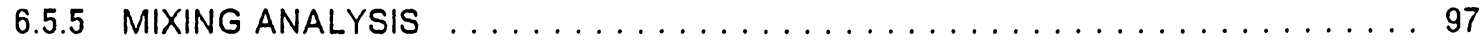

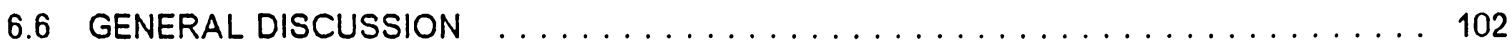


LOSS PRODUCTION MODELS $\ldots \ldots \ldots \ldots \ldots \ldots \ldots \ldots \ldots \ldots \ldots \ldots \ldots \ldots \ldots$

7.1 ADDITIONAL LOSSES DUE TO TIP LEAKAGE . . . . . . . . . . . . . . . . . . . . 104

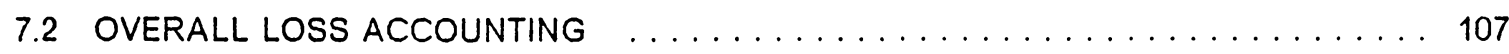

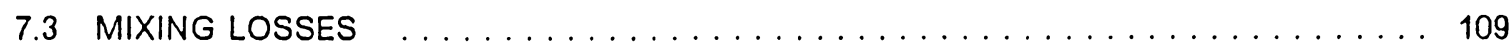

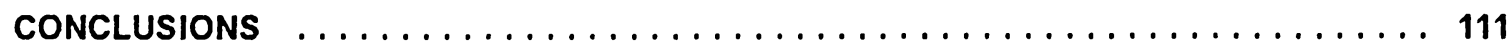

8.1 TIP LEAKAGE MEASUREMENTS $\ldots \ldots \ldots \ldots \ldots \ldots \ldots \ldots \ldots \ldots \ldots$

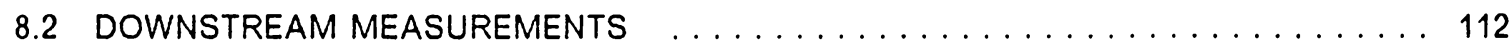

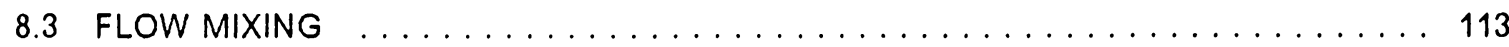

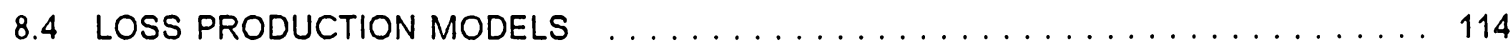

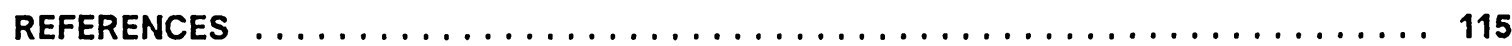

TIP LEAKAGE LOSS ESTIMATE $\ldots \ldots \ldots \ldots \ldots \ldots \ldots \ldots \ldots \ldots \ldots \ldots \ldots$

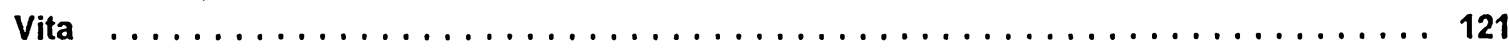




\section{List of Illustrations}

Figure 1. Roelke Correlation for Turbine Efficiency Loss due to Tip Leakage . . 4

Figure 2. Effects of Aerodynamic Design on Tip Leakage Losses . . . . . . . 7

Figure 3. Tip Gap Static Pressure Distribution $\ldots \ldots \ldots \ldots \ldots \ldots$

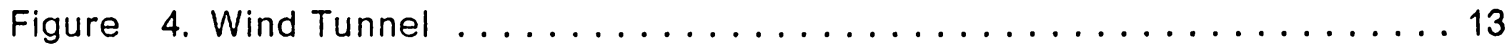

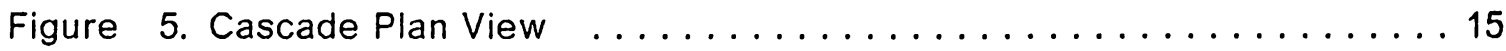

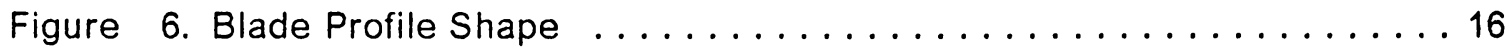

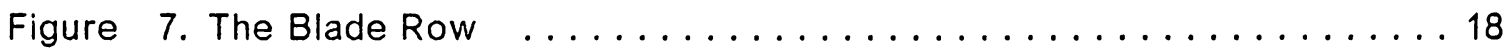

Figure 8. Blade Row and Tip Gap .................... 19

Figure 9. The Three Hole Cobra Probe $\ldots \ldots \ldots \ldots \ldots \ldots \ldots \ldots \ldots \ldots \ldots$

Figure 10. Five Hole Probe and Coordinate System $\ldots \ldots \ldots \ldots 22$

Figure 11. Bottom Endwall Flow Visualization $\ldots \ldots \ldots \ldots \ldots \ldots$

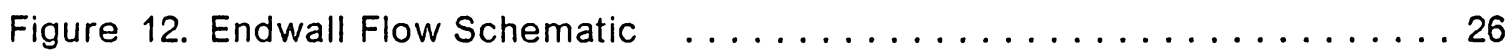

Figure 13. Pressure Surface Flow Visualization $\ldots \ldots \ldots \ldots \ldots \ldots$

Figure 14. Suction Surface Flow Visualization $\ldots \ldots \ldots \ldots \ldots$

Figure 15. Suction Surface and Endwall Visualization $\ldots \ldots \ldots \ldots \ldots$

Figure 16. Smoke Visualization of Tip Leakage Flow by Laser Light Sheet . . . 34

Figure 17. Smoke and Endwall Visualization of Tip Leakage Vortex in Flow Pas-

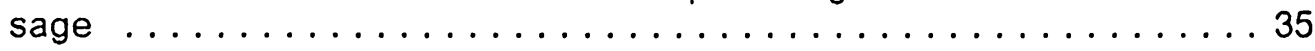

Figure 18. Tip Gap Probe Hole and Static Tapping Locations . . . . . . . . . 37

Figure 19. The Flow Passage and Downstream Measuring Plane .......41 
Figure 20. Tip Gap Variation Within Blade Row During Downstream Measurements .......................... 42

Figure 21. Tip Gap Total Velocity Profiles $\ldots \ldots \ldots \ldots \ldots$

Figure 22. Tip Gap Total Velocity Profiles $\ldots \ldots \ldots \ldots \ldots \ldots \ldots$

Figure 23. Tip Gap Exit Static Pressure Coefficients . . . . . . . . . . . . 49

Figure 24. Total Pressure Loss Coefficients at the Tip Gap Exit $\ldots \ldots \ldots$. . . 51

Figure 25. Total Pressure Loss Coefficients at the Tip Gap Exit $\ldots . \ldots . \ldots 52$

Figure 26. Flow Coordinate System at Tip Gap Exit Plane . . . . . . . . . . . 54

Figure 27. Flow Angle Distribution at the Tip Gap Exit $\ldots \ldots \ldots \ldots$

Figure 28. Flow Angle Distribution at the Tip Gap Exit $\ldots \ldots \ldots \ldots$

Figure 29. Normal Velocity Profiles . . . . . . . . . . . . . . . 57

Figure 30. Normal Velocity Profiles $\ldots \ldots \ldots \ldots \ldots \ldots$

Figure 31 . Streamwise Velocity Profiles $\ldots \ldots \ldots \ldots \ldots$

Figure 32. Streamwise Velocity Profiles $\ldots \ldots \ldots \ldots$

Figure 33. Discharge Coefficients at Tip Gap Exit Plane $\ldots \ldots \ldots \ldots \ldots$

Figure 34. Mass-averaged Total Pressure Loss Coefficients at the Tip Gap Exit

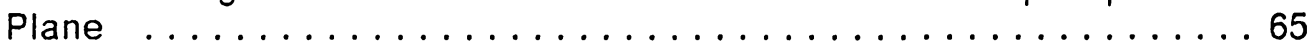

Figure 35. Tip Leakage Flow Model $\ldots \ldots \ldots \ldots \ldots \ldots$

Figure 36. Calculated and Measured Total Pressure Loss Coefficients $\ldots \ldots 72$

Figure 37 . Length to Tip Gap Height Ratios $\ldots \ldots \ldots \ldots \ldots$

Figure 38. Cross-Sectional Plane and Coordinate System . . . . . . . 76

Figure 39. Secondary Velocity Vectors in the Cross-sectional Plane at $x / c=1.477$

Figure 40. Contours of Constant Total Pressure Loss Coefficient $\ldots \ldots \ldots$

Figure 41. Secondary Velocities and Total Pressure Loss Coefficients . . . . . 80

Figure 42. Contours of Constant Static Pressure Coefficient $\ldots \ldots \ldots$. . . 82

Figure 43. Flow Coordinate System at Downstream Measuring Plane . . . . . 84

Figure 44. Pitch-wise Averaged Total Pressure Loss Coefficient, Cpta . . . . . 93 
Figure 45. Pitch-wise Averaged Total Pressure Loss Coefficient, Cptb . . . . . 94

Figure 46. Span-wise Averaged Total Pressure Loss Coefficient, Cptc . . . . . 995

Figure 47. Span-wise Averaged Total Pressure Loss Coefficient, Cptd . . . . . 96

Figure 48. Pitch-wise-mass-averaged Flow Angle $\ldots \ldots \ldots \ldots \ldots \ldots$

Figure 49. Mixing Control Volume $\ldots \ldots \ldots \ldots \ldots \ldots \ldots \ldots . \ldots . \ldots 9$

Figure 50. Turbine Configuration and Geometry $\ldots \ldots \ldots \ldots \ldots \ldots \ldots$ 


\section{List of Tables}

Table 1. Tip Gap Exit Static Tapping and Probe Hole Locations . . . . . . . . . 38

Table 2. Tip Gap Measurement Planes and Tip Gap Heights . . . . . . . . . . 39

Table 3. Averaged Flow Properties ..................... 89

Table 4. Mixed Out Flow Properties . . . . . . . . . . . . . . . . 101

Table 5. Comparison of Tip Leakage Cascade with Non-leakage Cascade . . . 102

Table 6. Comparison of the Top and Bottom Halves of the Flow Passage . . . 110- 


\section{Nomenclature}

A flow passage area

$C_{D}$ discharge coefficient, Eq. 6.3

$C_{p s} \quad$ static pressure coefficient, Eq. 6.2

$C_{p t} \quad$ total pressure loss coefficient, Eq. 6.1

$\mathrm{H}_{12} \quad$ shape factor

$P \quad$ static pressure $\left(\mathrm{N} / \mathrm{m}^{2}\right)$

$P_{\text {so }} \quad$ cascade inlet static pressure $\left(\mathrm{N} / \mathrm{m}^{2}\right)$

$P_{t} \quad$ total pressure $\left(\mathrm{N} / \mathrm{m}^{2}\right)$

$P_{\text {to }} \quad$ cascade inlet total pressure $\left(\mathrm{N} / \mathrm{m}^{2}\right)$

$Q \quad$ passage volume flow rate $\left(\mathrm{m}^{3} / \mathrm{s}\right)$

SKE secondary kinetic energy

$S_{b} \quad$ tip gap exit length

$u$ velocity component in primary flow direction $(\mathrm{m} / \mathrm{s})$

$U_{0} \quad$ cascade inlet free stream velocity $(\mathrm{m} / \mathrm{s})$

$u_{n} \quad$ tip gap normal velocity $(\mathrm{m} / \mathrm{s})$

$u_{s} \quad$ tip gap streamwise velocity $(\mathrm{m} / \mathrm{s})$

$U_{t} \quad$ tip gap exit velocity based on potential flow $(\mathrm{m} / \mathrm{s})$

$v \quad$ velocity component orthogonal to $u$ and $w(\mathrm{~m} / \mathrm{s})$

$V \quad$ velocity at downstream measurement plane $(\mathrm{m} / \mathrm{s})$

$V_{n} \quad$ velocity component normal to downstream measuring plane $(\mathrm{m} / \mathrm{s})$

w velocity component in spanwise direction

$x / c \quad$ axial distance from blade leading edge/axial chord

$\Delta Y \quad$ blade pitch

$\Delta Z \quad$ blade span 


\section{Greek Letters}

$\alpha \quad$ tip gap flow angle $\left({ }^{\circ}\right)$, Fig. 26

$\beta \quad$ flow angle with respect to pitchwise direction $\left({ }^{\circ}\right)$

$\delta_{99} \quad$ boundary layer thickness $(\mathrm{mm})$

$\delta \star \quad$ displacement thickness

$\delta_{t} \quad$ tip gap height

$\ell / \delta_{\mathrm{t}} \quad$ leakage flow path/tip gap height

$\theta \quad$ momentum thickness, flow angle with respect to axial direction $\left({ }^{\circ}\right)$

$\rho \quad$ fluid density $\left(\mathrm{kg} / \mathrm{m}^{3}\right)$

\section{Subscripts}

0 upstream of cascade, reference

1 cascade inlet.

2 cascade exit

d mixed out plane

ex tip gap exit plane

$n$ normal

$s \quad$ streamwise, static conditions

$t \quad$ total conditions

$x \quad$ axial direction

y pitchwise direction

\section{Superscripts}

- area average, average

$=$ mass average 


\section{Chapter 1}

\section{INTRODUCTION}

Performance improvement in turbomachines is dependent upon an understanding of loss production mechanisms. At present this understanding is primarily gained through experimental means requiring detailed flow measurements. If the nature of the flow losses, how and where they are produced, can be determined, then techniques for designing more efficient turbomachinery can be developed. One loss mechanism which is not yet adequately understood is tip leakage flow.

Tip leakage flow is a result of blade loading. The pressure difference across the blade tip drives a portion of the passage flow through the clearance gap between the rotor tip and casing. This secondary flow of fluid from the pressure to the suction side of a blade degrades the performance of turbomachines by two distinct mechanisms. First, the amount of work done per blade is reduced due to underturning of the flow near the tip. Second, as the leakage flow exits the tip gap it rolls up into a vortex which creates additional losses as it mixes out into the primary flow. 
This thesis presents the results of an experimental study of tip leakage flow in a linear turbine cascade. It further explains the details of flow from the tip gap and then relates this to loss production downstream of the blade row. Extensive experimental data has been accumulated on the VPI \& SU turbine tip leakage cascade by both this study and the earlier work of Moore and Tilton [ 1].

In addition to enhancing the understanding of tip leakage flow and its effects on turbine performance, the work on this cascade will act as a test case for three dimensional viscous flow calculations being developed for turbine rotor flow calculations and turbine stage design. 


\section{Chapter 2}

\section{LITERATURE REVIEW}

It is generally agreed that tip leakage flow has significant detrimental effects on turbine performance. The loss in efficiency and reduction of work due to tip leakage have been well documented [ 2,3,4,5]. Recently, it has been suggested that tip leakage flow is a major contributing factor to turbine tip burnout [ 1,6 ]. The full extent of the effects of tip leakage flow, however, is not yet understood. Bindon [ 7 ] suggests that this is because, "most research has concentrated on the problem of loss prediction and reduction rather than on the basic flow mechanisms."

\subsection{LOSS PREDICTION AND REDUCTION}

Roelke [ 8 ] published a correlation for the decrease in turbine efficiency with increased tip clearance, see Figure 1. 


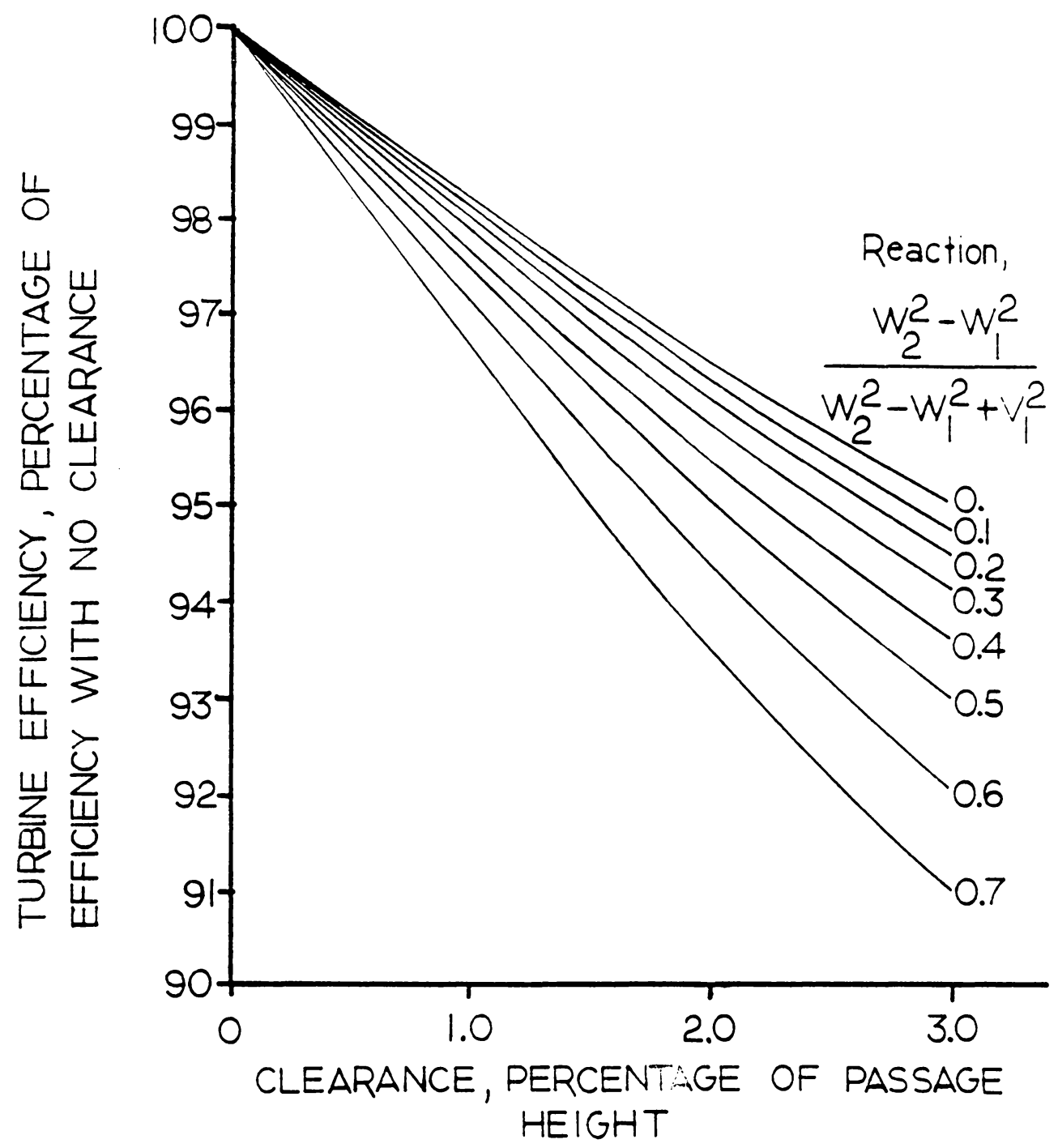

Figure 1. Roelke Correlation for Turbine Efficiency Loss due to Tip Leakage 
In general, a $2 \%$ drop in efficiency is predicted for each $1 \%$ rise in clearance as a percentage of the passage height. The degree of turbine reaction also seems to be a factor. The higher the reaction, the greater the exchange of efficiency for tip clearance. The data for his correlation was taken from earlier NASA publications [ 2,3,4] and other works which were traced back to a Russian source [ 9 ]. An estimate of the losses in the VPI \& SU tip leakage cascade using Roelke's correlation is made in Appendix A.

Methods to reduce the detrimental effects of rotor tip clearance have been investigated using both mechanical and aerodynamic design techniques. Hennecke [ 10 ] distinguishes between active and passive mechanical systems for the control of rotor blade tip clearances. Active systems allow the tip clearance to be set and maintained at a prescribed value while running. This normally involves heating or cooling of the rotor or casing to control the temperature and, therefore, the thermal growth of each component. This enables the desired tip clearance to be maintained. Passive systems, on the other hand, do not allow active control of the tip clearance. They are designed to equalize the thermal response of both the rotor and the casing so that the tip clearance remains relatively constant. This involves slowing the thermal response of the casing and hastening that of the rotor. The addition of mass to the casing or the use of a double walled casing can achieve the former although with a considerable weight penalty. The response of the rotor can then be increased by sending hot vent air through the disk and hub. These methods of mechanical control are effective in decreasing losses due to tip leakage but not without the penalty of added complexity and additional sources of inefficiency.

Aerodynamic design of blade tips and casing has been reported to decrease the loss in turbine efficiency due to tip leakage [2,3,11]. Many tip configurations such as winglet, squealer, groove and knife edge have been tested with varying 
degrees of success. Shrouding and casing contouring have also been suggested as a means of tip leakage control. The prime objective of these techniques is to increase the resistance to flow through the tip gap and reduce the discharge coefficient.

Hourmouziadis and Albrecht [ 12 ] shed doubt upon the present methods of aerodynamically dealing with the tip leakage problem. In their tests, they could not confirm the effectiveness of several of the above mentioned methods. They found no effect on the overall loss level with the winglet tip configuration or from recessing the casing with circumferential grooves. These tests were not totally comprehensive but they did "cover a large variety of pressure ratios, aspect ratios and reaction." The only consistency throughout the test was the rate of exchange of efficiency for tip clearance, about 2\% efficiency for each 1\% clearance, see Figure 2.

The work of Hourmouziadis and Albrecht questions the present aerodynamic methods of combatting tip leakage flow as well as the ability to predict its resulting losses. In turn, it brings into question the present understanding of the fluid mechanics and loss production mechanisms of tip leakage flow.

\subsection{WORK TO DATE}

Most of the experimental work directed toward understanding the fundamental fluid mechanics of tip leakage flow has concentrated on the flow while it is still within the tip gap. Bindon, in companion reports [ 6,7,13 ], looks at tip leakage flow through visualization and measurement techniques. He finds a microscopic region of ultra low pressure at the pressure surface corner of the blade. This is associated 


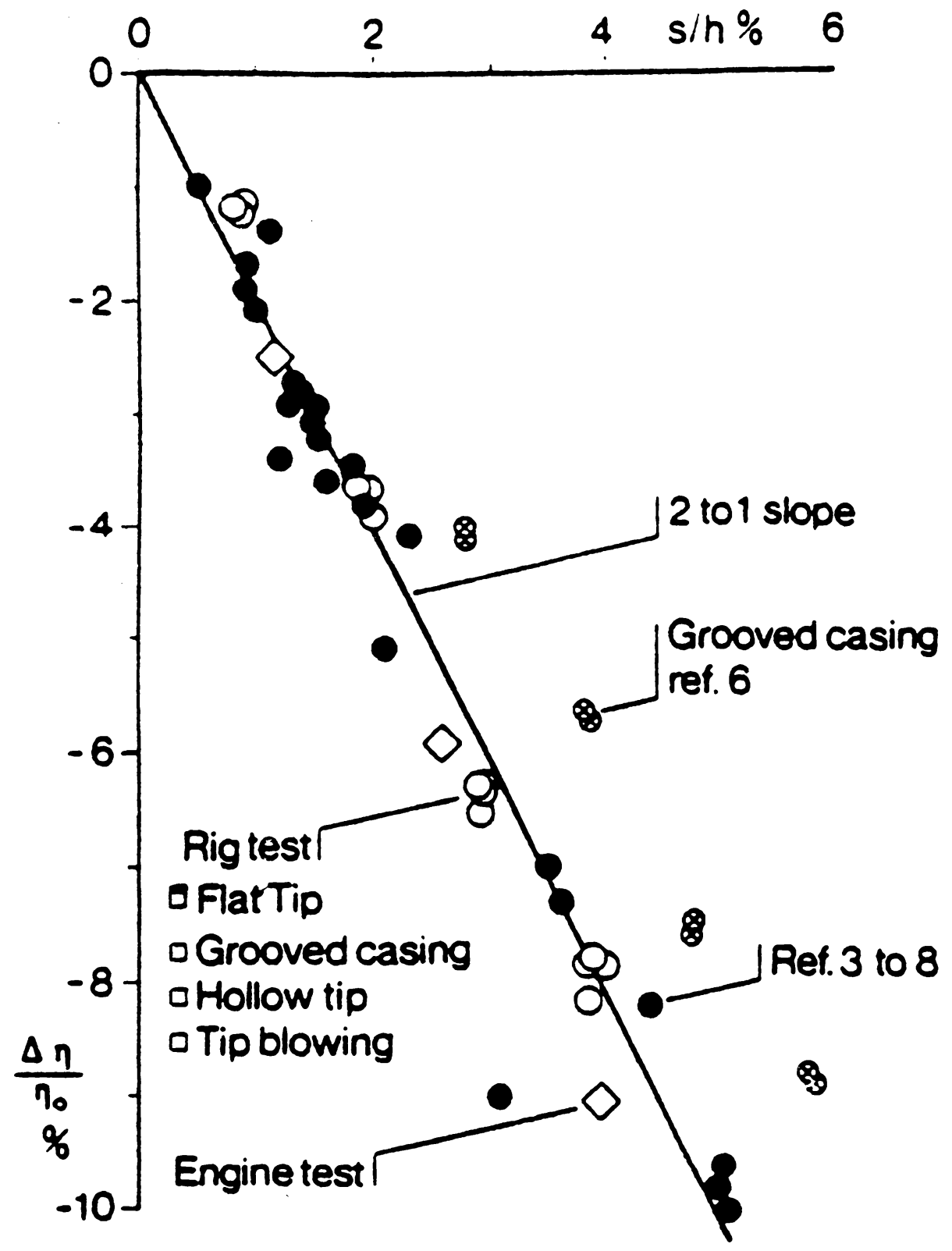

Figure 2. Effects of Aerodynamic Design on Tip Leakage Losses: Hourmouziadis and Albrecht [12] 
with the high velocity demanded by the small radius through which the leakage flow must turn as it goes under the blade. He is surprised by this low pressure because the separation bubble which forms under the blade tip should increase the radius of curvature and therefore lessen the pressure reduction. He concludes that the flow remains attached around the pressure corner, not forming a separation bubble until slightly into the tip gap.

Bindon also finds a deep low pressure region near the suction surface of the blade. This region, he believes is a result of the curvature of the main flow as it is rerouted around the leakage vortex. This low pressure, in turn, reinforces the leakage flow.

In addition, Bindon presents flow measurements made in the tip gap exit plane as well as others in the passage between the blades. Over the forward half of the blade, the leakage flow is of a low loss level and has little effect on the primary passage flow. Near mid-axial chord, the loss level of the leakage flow rises sharply. The effects of the leakage jet also become apparent in the passage flow at about mid-axial chord. The influence of the leakage is shown to grow until the blade row exit plane, after which no data was taken. Compared to his measurements with no tip clearance, the mass-averaged loss at the blade row exit plane for the tip clearance case is twice as great although the overall area encompased by loss is about the same.

Moore and Tilton [ 1 ] used static pressure measurements on both the blade tip and the endwall under the blade tip to study tip leakage flows. As seen in Figure 3 , the static pressure along the endwall falls rapidly near the pressure surface of the blade indicating acceleration into the tip gap.

This acceleration continues until about 2 tip gap heights into the tip gap where both the endwall and blade tip static pressures begin to rise. They equalize by about 4 tip 


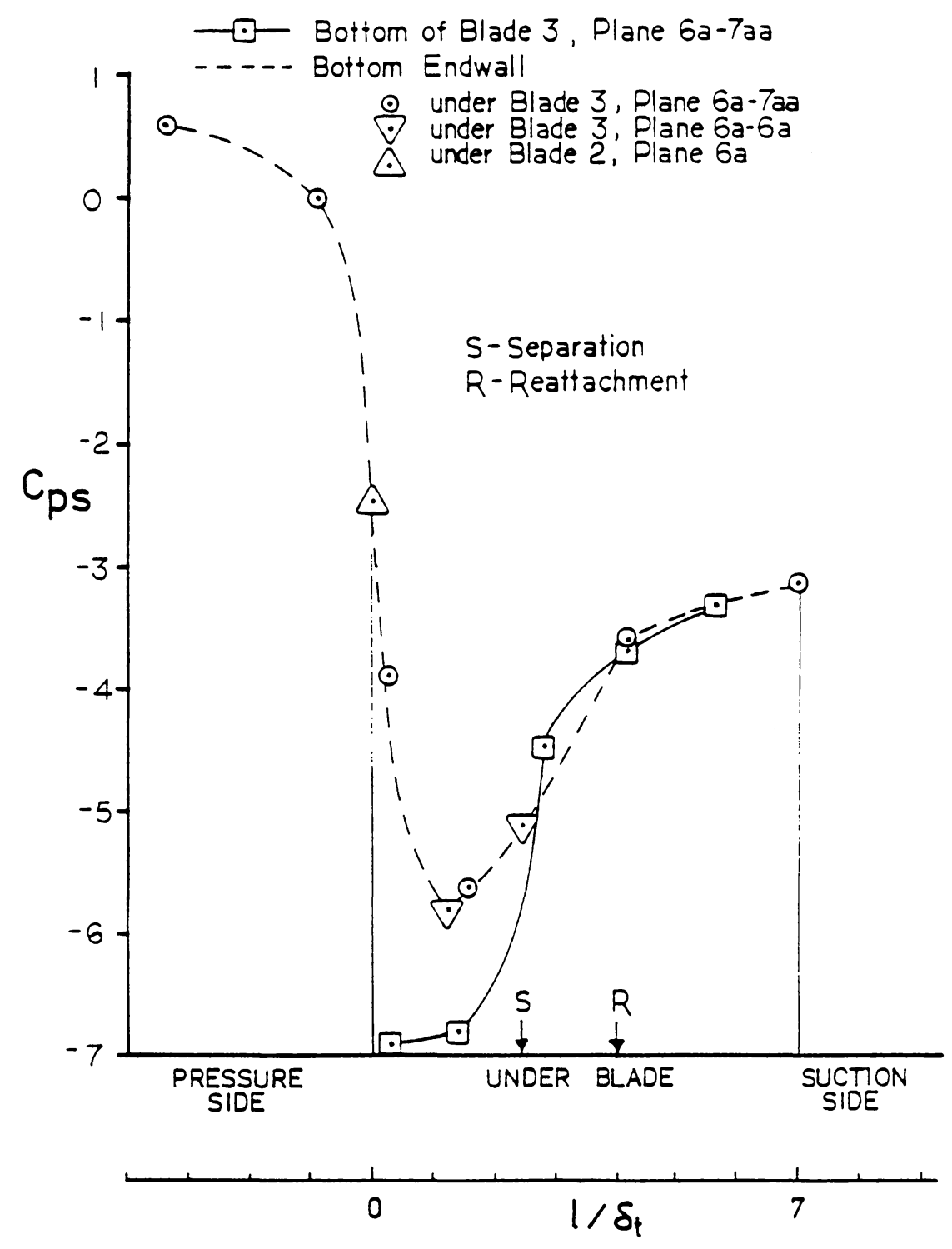

Figure 3. Tip Gap Static Pressure Distribution: Moore and Tilton [1] 
gap heights and remain so up to the tip gap exit. This pressure distribution indicates the presence of a vena contract at the point of minimum endwall static pressure followed by flow mixing.

From their observations, Moore and Tilton were able to devise a mathematical model for predicting tip gap total pressure losses due to flow mixing after the vena-contracta. The effectiveness of the model was tested with data provided by Bindon [ 7 ] and it was found to be quite accurate. The details of this model and its prediction of the tip gap losses for the present cascade are given in section 6.4.

\subsection{OTHER WORK}

Various studies of tip leakage and the factors affecting it have been performed. Klein [ 14] studied the effects of boundary layer thickness, inlet skew and relative motion between blades and endwall on the magnitude and distribution of tip leakage losses for various tip clearances.

Sjolander and Amrud [ 15 ], in studying the effects of tip leakage on blade loading reported the appearance of multiple tip leakage vortices as clearance was increased. Many other works have been reported but there is still a need for a study which relates flow from the tip gap to loss production downstream of the blades. 


\subsection{PRESENT STUDY}

The objective of this study is to investigate details of the leakage flow as it exits the tip gap and then follow it downstream. Moore and Adhye [ 16 ] discovered that over one third of the losses in their turbine cascade occurred downstream of the blade trailing edge. More importantly, they observed that the downstream losses were almost entirely explained by the dissipation of secondary kinetic energy. Tip leakage results in large secondary flows and kinetic energies. Therefore, it is likely to be a major contributor to the losses produced downstream. This study provides an understanding of tip leakage losses and how they develop. 


\section{Chapter 3}

\section{APPARATUS}

Most of the experimental apparatus used in this study is the same as that used previously by Moore and Tilton [ 1]. Therefore, only the modifications and additions will be explained in detail.

\subsection{WIND TUNNEL AND CASCADE}

The $20.4 \mathrm{~kW}$ blower configuration wind tunnel used for this study is shown along with the cascade in Figure 4.

After the flow exits the blower it is diffused and straightened. The flow is then accelerated through a nozzle into the cascade. Between the nozzle and the test section is a short inlet duct and a pair of endwall boundary layer bleeds. The endwall boundary layers were removed prior to the test section in order to reduce the sec- 

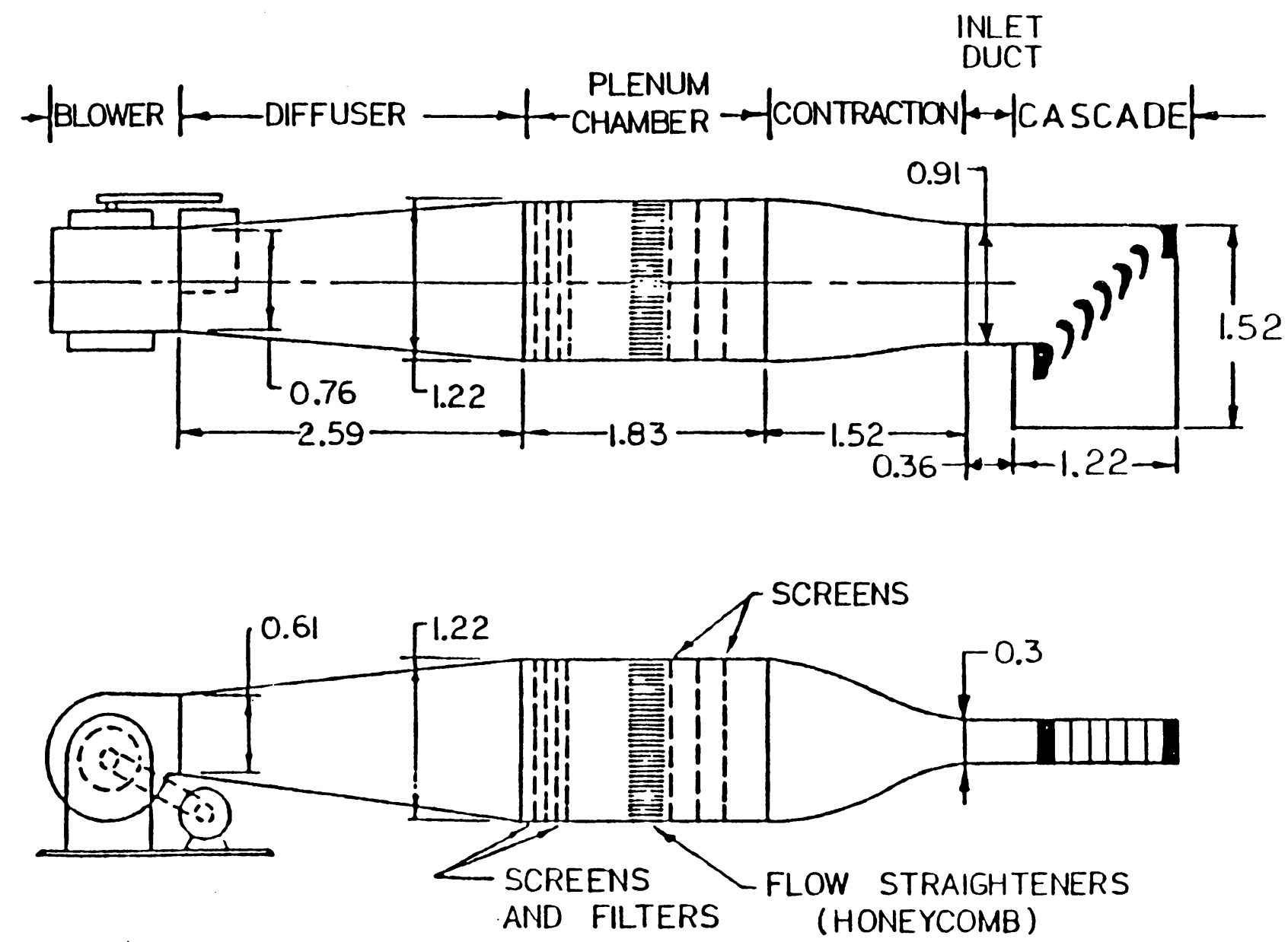

Figure 4. Wind Tunnel: All dimensions in meters 
ondary flows they produce within the blade passages. Thus, the effects of the tip leakage flow are not obscured by other three dimensionalities. In addition, a 0.51 $\mathrm{mm}$ diameter trip wire is located near the leading edge of each bleed to insure that the new developing boundary layer is turbulent and uniform.

Figure 5 shows a plan view of the cascade which includes the bottom endwall boundary layer bleed.

Also shown are the two adjustable end bleeds used to maintain flow repeatability within the cascade. The cascade endwalls are plywood, lined with Formica to create a smooth surface. The side walls are also Formica lined plywood with the exception of two sections of clear plexiglas for viewing.

\subsection{THE BLADE ROW AND TIP GAP}

The individual blades were designed to be geometrically similar to those used by Langston [ 17 ]. The profile shape is shown in Figure 6.

The blade shape is that of a reaction turbine having mean camber line angles $\beta_{1}=43.99^{\circ}$ and $\beta_{2}=25.98^{\circ}$ measured relative to the blade to blade direction. The blades produce a velocity ratio of 1.6 through a nominal turning angle of $110^{\circ}$. The overall blade dimensions are given below.

$$
\begin{aligned}
\text { Axial Chord } & =235.2 \mathrm{~mm} \\
\text { Span } & =234.4 \mathrm{~mm} \\
\text { Pitch } & =224.8 \mathrm{~mm}
\end{aligned}
$$




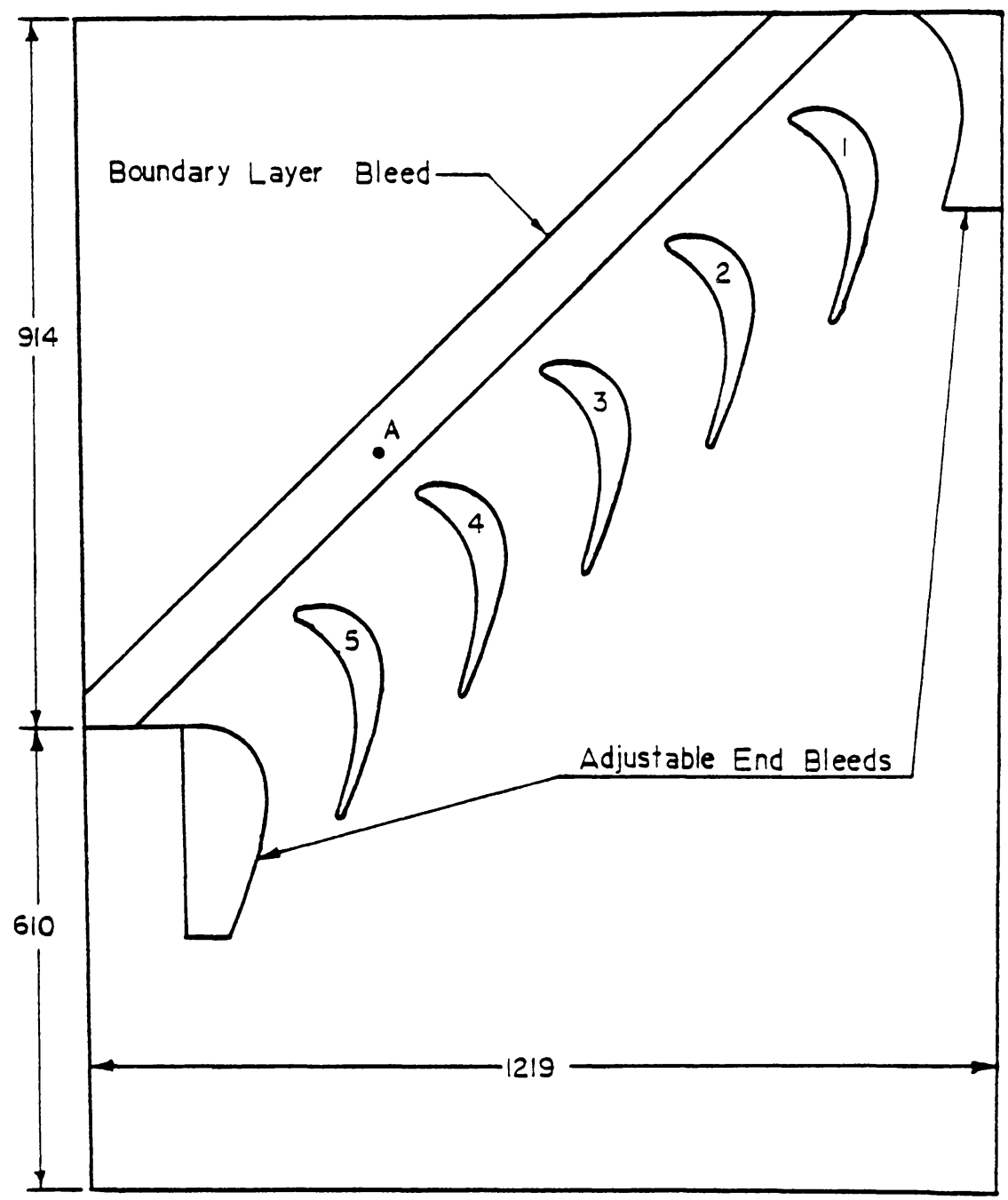

Figure 5. Cascade Plan View: All dimensions in $\mathrm{mm}$ 


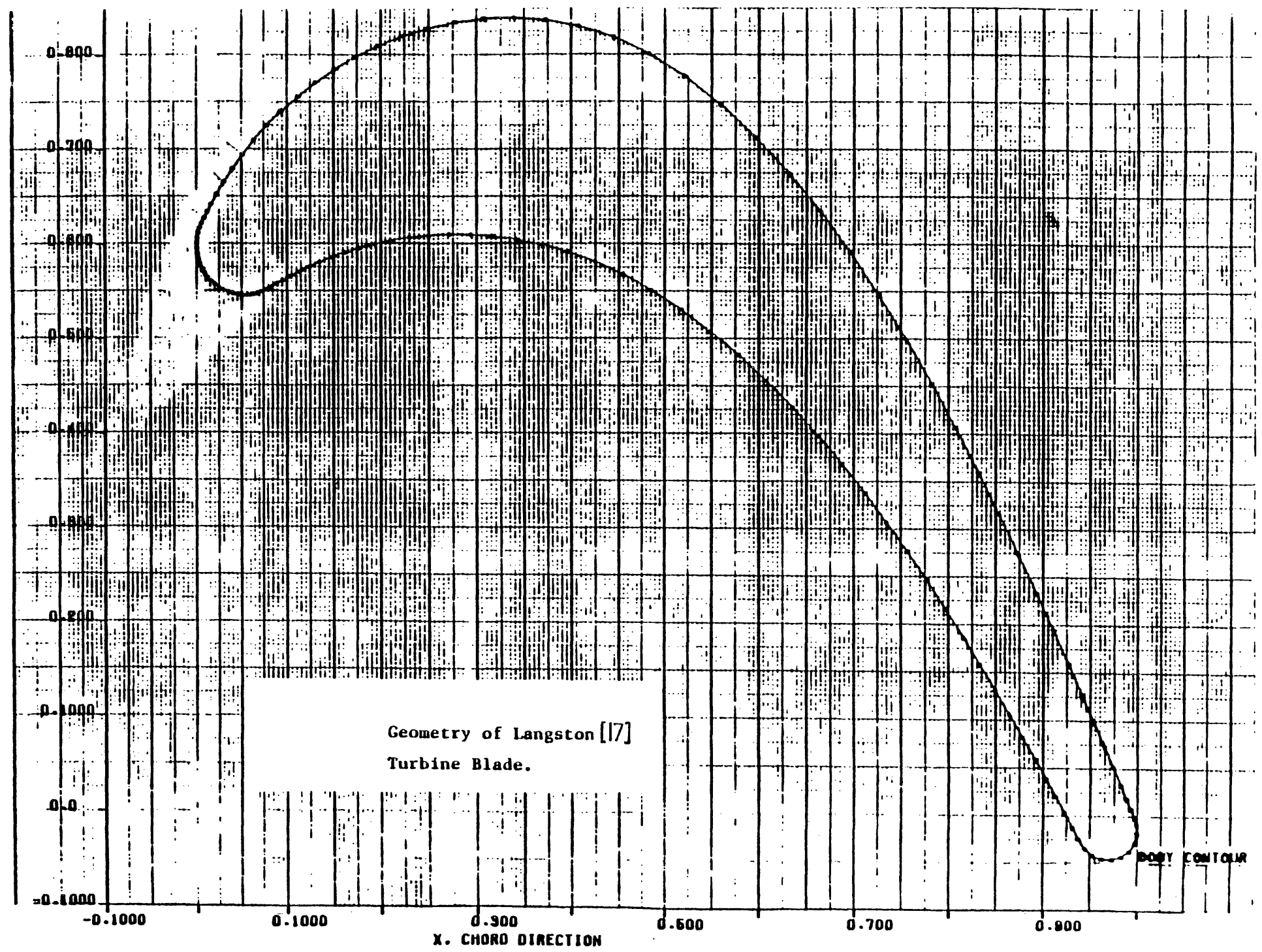


Five such blades and the two end bleeds form the six passage blade row which is shown in Figure 7.

Also shown are the blade numberings, blade dimensions, and air inlet and exit angles. In order to start uniform turbulent boundary layers on the blade surfaces, trip wires were located downstream of the leading edge on both the pressure and suction surfaces.

The tip gap is nominally $5 \mathrm{~mm}, 2.1 \%$ of the blade span, giving a passage height of $239.4 \mathrm{~mm}$. The tip gap varied slightly through the blade row but never by more than $0.1 \%$ of the blade span in either direction. Detailed measurements of the tip gap height taken during different phases of this study will be presented later.

A portion of the blade row showing the tip gap viewed from upstream is seen in Figure 8.

Also visible are the previously described endwall boundary layer bleeds.

\subsection{MEASURING EQUIPMENT}

All measurements in this study were made with pressure probes and static pressure tappings. The pressure signals were converted to electrical signals with pressure transducers. Bridge amplifiers boosted the signals which were then monitored on an. oscilloscope. The output was converted to a pressure reading with previously determined transducer calibration curves. The calibration curves were verified with an inclined meriam oil manometer prior to any recording of data. 


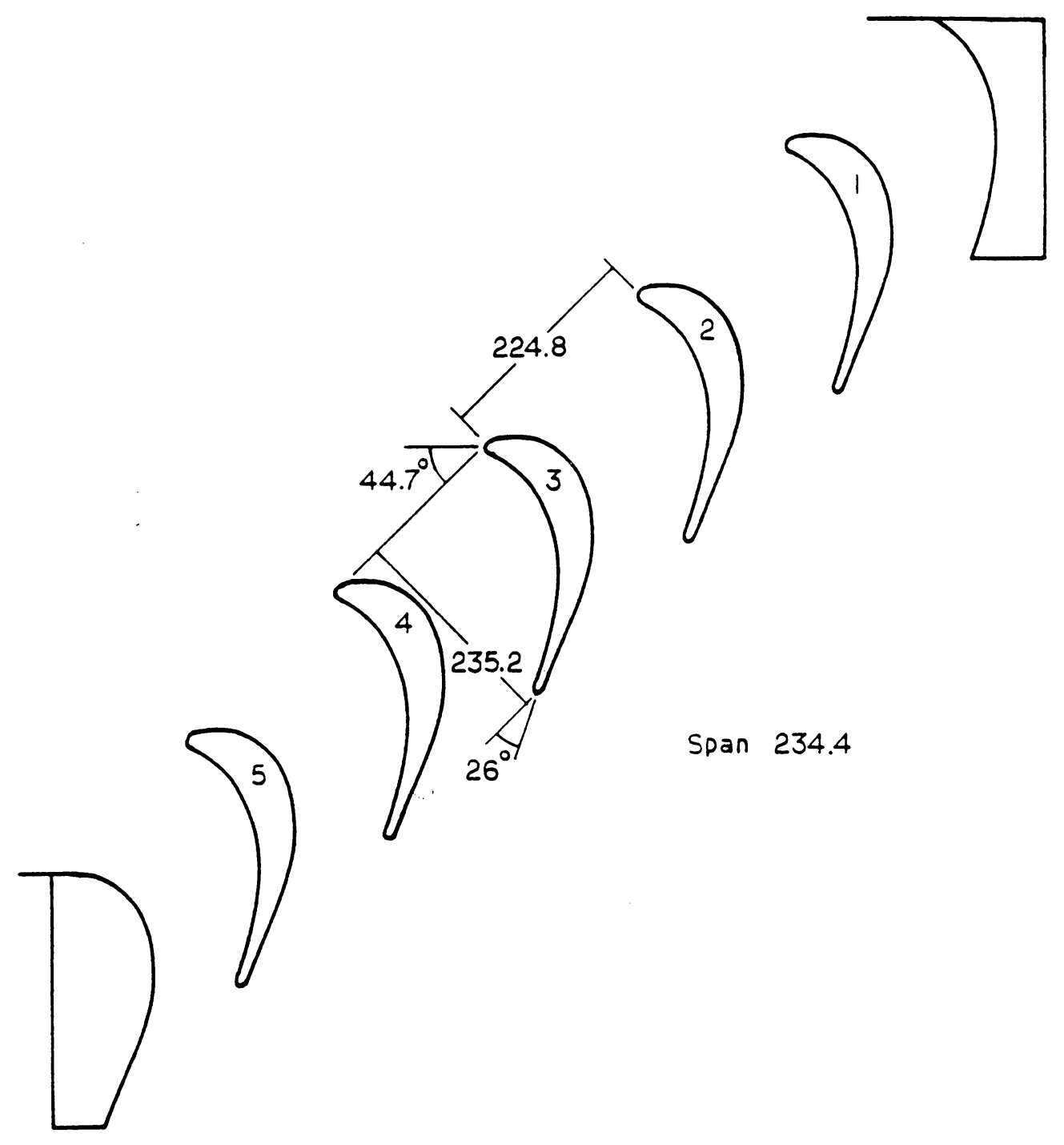

Figure 7. The Blade Row: All dimensions in $\mathrm{mm}$ 


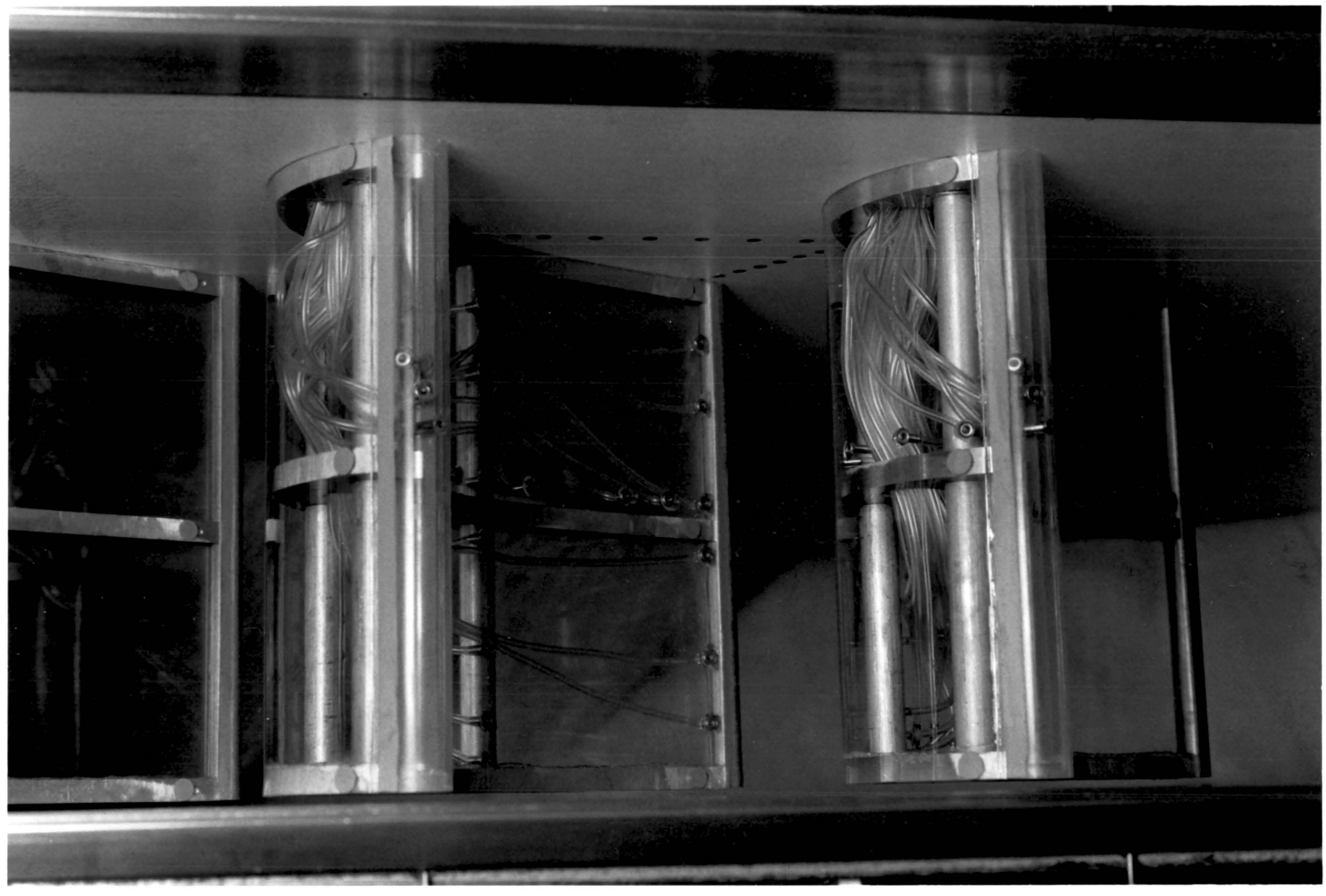

Figure 8. Blade Row and Tip Gap 
Four pressure probes were used in this study. A total pressure probe inserted in instrumentation port A of Figure 5 and a static pressure tap located upstream of blade 3 were used to measured the cascade inlet conditions.

The tip gap measurements were made with a three-hole cobra probe constructed for this study. The probe and its dimensions are shown in Figure 9.

A simple traversing gear which allowed spanwise movement and probe rotation was used for these measurements. The probe positioning was done by hand. A dial gage readable to $0.01 \mathrm{~mm}$ was used to measure spanwise position during each traverse.

Cascade measurements downstream of the blade row were made with a United Sensor five-hole probe having a $3.18 \mathrm{~mm}$ ( $1 / 8 \mathrm{in}$. ) tip diameter. The probe was calibrated by Ransmayr [ 18 ] and again by Adhye [ 19 ] to read total and static pressures as well as the three velocity components, $u, v$, and $w$. The probe tip and its measurement coordinate system are shown in Figure 10.

The five-hole probe was positioned by a traversing gear which was mounted above the cascade. This allowed pitchwise and spanwise traverses, the locations of which were determined with calibrated dial counters. The traversing gear also provided rotation allowing the probe to be nulled in yaw.

Because of its size, the five-hole probe could not be used near the passage endwalls. Therefore, the tip leakage probe and an additional but longer three-hole probe were used in these areas. The traversing apparatus was the same as previously described for the tip leakage probe.

The static pressure tappings were made from $6.35 \mathrm{~mm}$ diameter brass rod with $1.02 \mathrm{~mm}$ holes drilled through their center. The tappings were inserted through holes in the endwall and made flush with the surface so as to cause no unnecessary flow disturbances. 


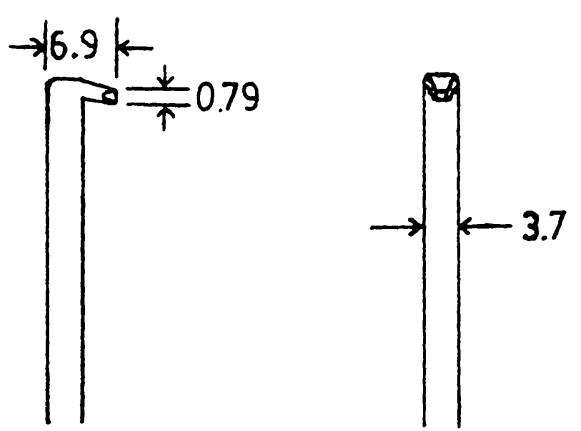

Figure 9. The Three Hole Cobra Probe: All dimensions in $\mathrm{mm}$ 


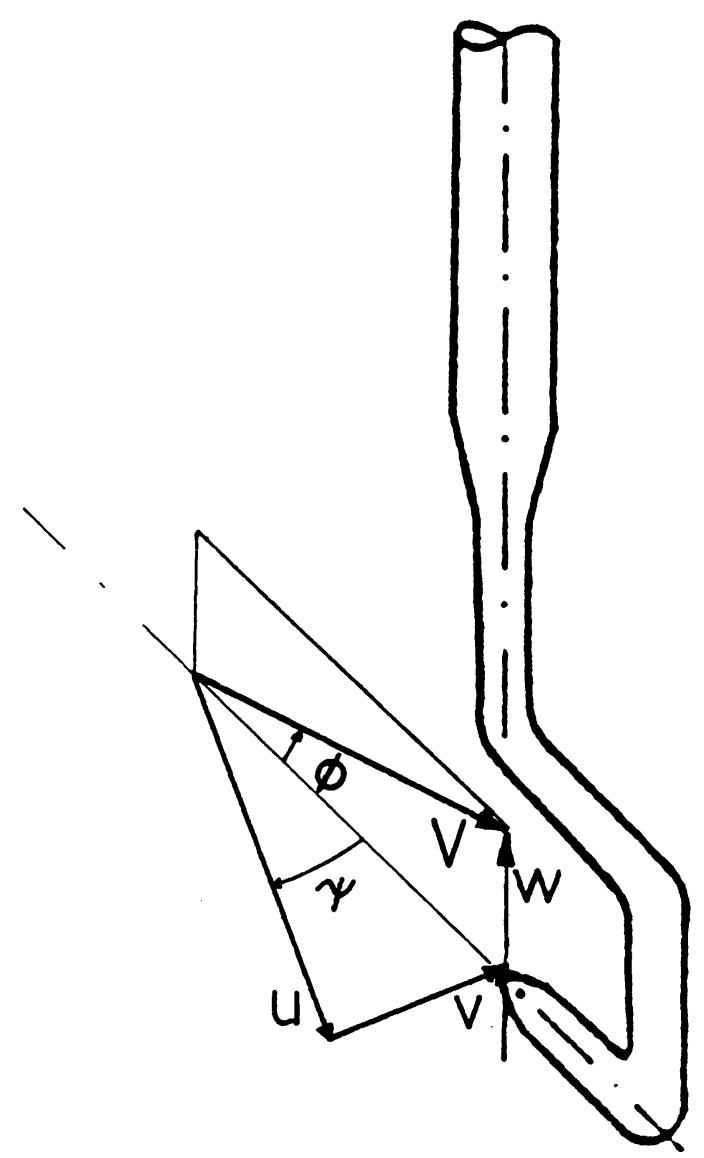

Figure 10. Five Hole Probe and Coordinate System 


\section{Chapter 4}

\section{FLOW VISUALIZATION}

A flow visualization study was performed to gain a qualitative understanding of the tip leakage flow. Aside from the descriptive insight gained, the visualizations were helpful in planning the quantative flow measurements.

\subsection{SURFACE VISUALIZATIONS}

Surface flow visualizations were done on the suction surface of blade 3 , the pressure surface of blade 2, and on the bottom endwall between the two blades. Sheets of $0.09 \mathrm{~mm}$ thick Mylar painted with Krylon Ultra-Flat Black Enamel were taped to the surfaces. They were then painted with a mixture made from 10 grams of finely filtered titanium dioxide, $40 \mathrm{ml}$ of diesel oil and 2 drops of oleic acid. The wind tunnel 
was run for approximately 30 minutes allowing the mixture to dry. The visualizations were then removed and protected with Krylon Crystal Clear Acrylic spray coating.

\subsubsection{BOTTOM ENDWALL}

The endwall visualization shown in Figure 11 was made earlier by Moore and Tilton [1].

It is presented here because it shows the locations of the blade tips and it encompasses a larger flow area than the visualization done in the present study. Being done on the same cascade, both visualizations provide the same qualitative information. The more recent visualization will be shown later in conjunction with the blade surface visualizations.

The details of the endwall visualization are illustrated more clearly in the schematic of Figure 12.

The turbulent inlet boundary layer splits at point $L$, but without rolling up into a horseshoe vortex as it would at the blade root where no clearance gap is present. Passage fluid from near the pressure surface blade tip flows toward the endwall, attaching as a laminar boundary layer and then diverging along $\mathrm{A} 1$ [ 1 ]. Downstream of $L 1$, the flow accelerates under the blade to a laminar separation at S3. It then reattaches as a turbulent boundary layer at R1. The flow procedes through the tip gap, separating again at $\mathrm{S} 1$ and rolling up into a leakage vortex.

Secondary flow, including fluid from the inlet boundary layer ( upstream of $L 2$ ) and from the passage ( attaching along $A 1$ downstream of $L 2$ ), meets the leakage flow and separates along S2. The separation lines S1 and S2 move farther from the blade suction surface as the leakage jet gains strength downstream of the 


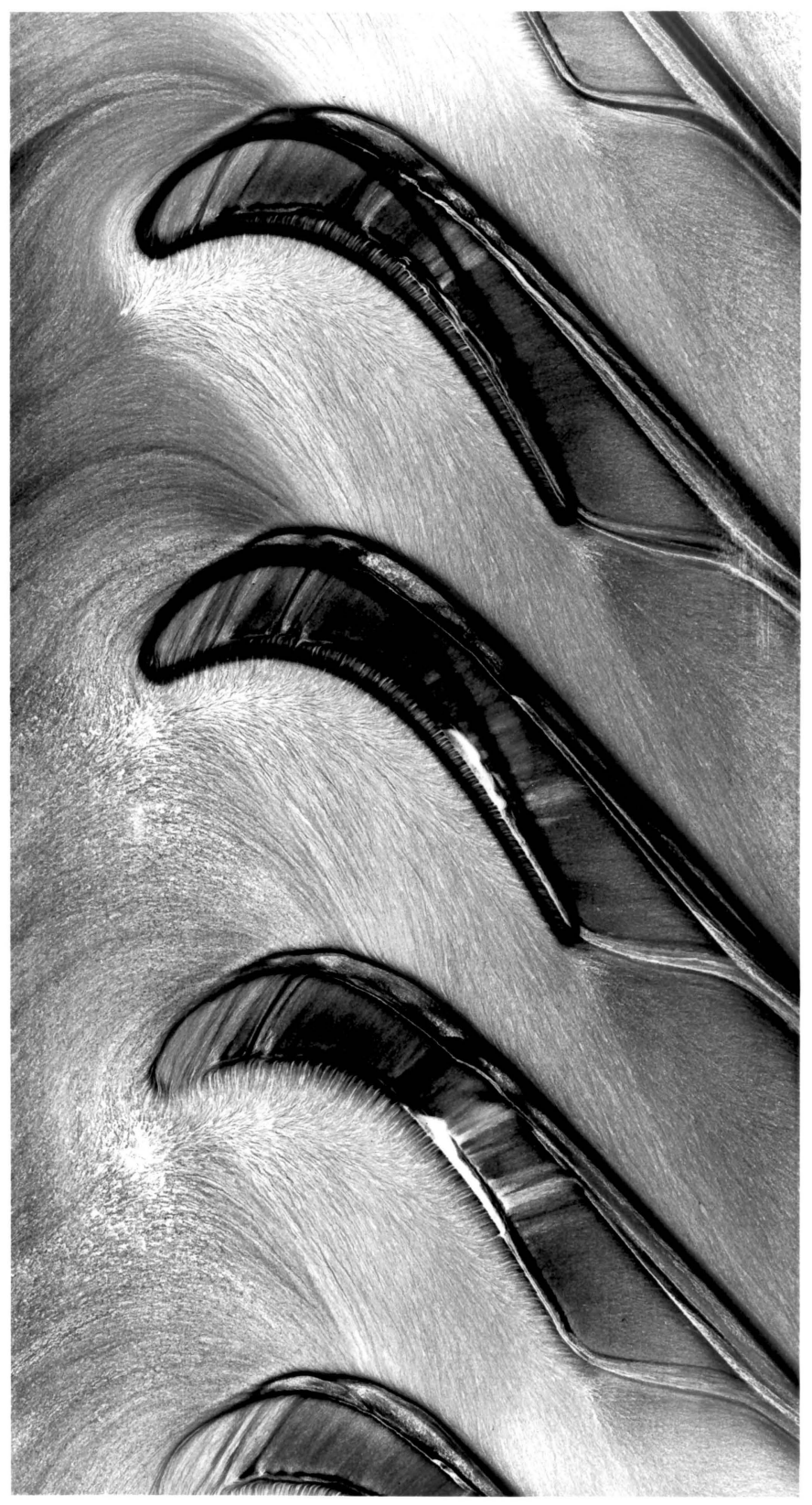

Figure 11. Bottom Endwall Flow Visualization: Moore and Tilton [ 1 ] 
$\frac{n}{5}$
$\frac{1}{5}$
$\frac{5}{2}$
$\frac{1}{N}$
$\frac{1}{2}$
$\frac{1}{2}$

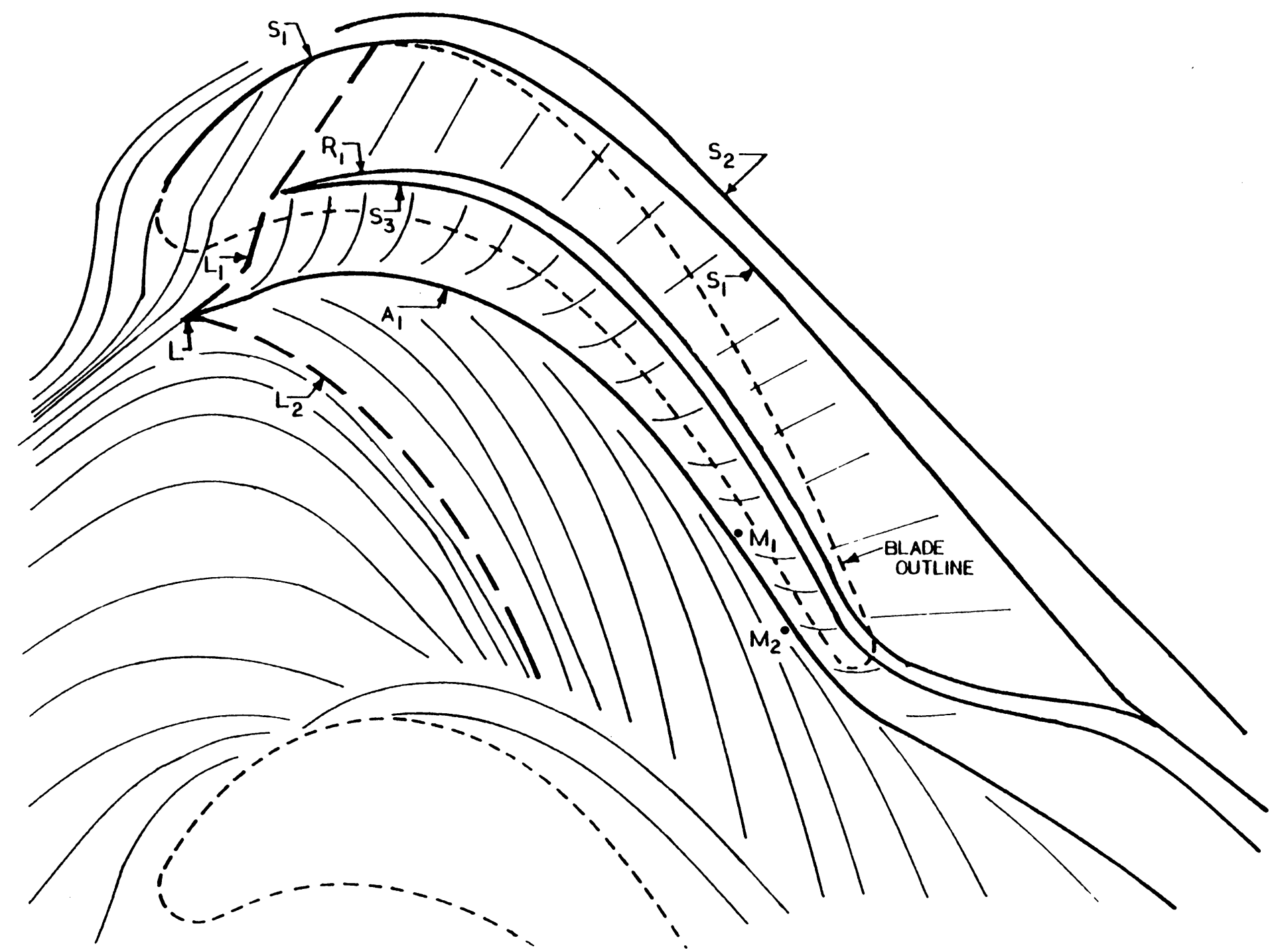

Figure 12. Endwall Flow Schematic: Moore and Tilton [ 1 ] 
leading edge. Note that upstream of $L 1$ the leakage flow is very weak, separating at S1 when it meets the passage flow prior to exiting the tip gap.

Downstream of the blade row, as the leakage vortex dissipates, separation continues along S1 and S2. The downstream continuation of R1 and S3, however, is suspected of being the trace of residual visualization fluid rather than an actual flow phenomenon.

\subsubsection{PRESSURE SURFACE}

A portion of the pressure surface visualization from $x / c=0.69$ to $x / c=0.87$ is presented in Figure 13.

The bottom half of the blade is seen from tip to $45 \%$ span with the passage flow going from left to right. The endwall visualization is included to create a more complete picture and to better illustrate the flow's behavior near the tip gap.

Evidence of the flow's acceleration into the tip gap is clearly visible. The downward turning of the passage flow near the pressure surface is seen to extend about 3 tip gap heights above the endwall. Also visible are the traces from the passage flow on the bottom endwall as it attaches at A1 and sweeps under the blade tip.

\subsubsection{SUCTION SURFACE}

An earlier visualization of the entire suction surface is shown in Figure 14. It presents clear evidence of both a leakage vortex near the blade tip and a passage vortex near the root. Near mid-span, however, there seems to be little effect from 


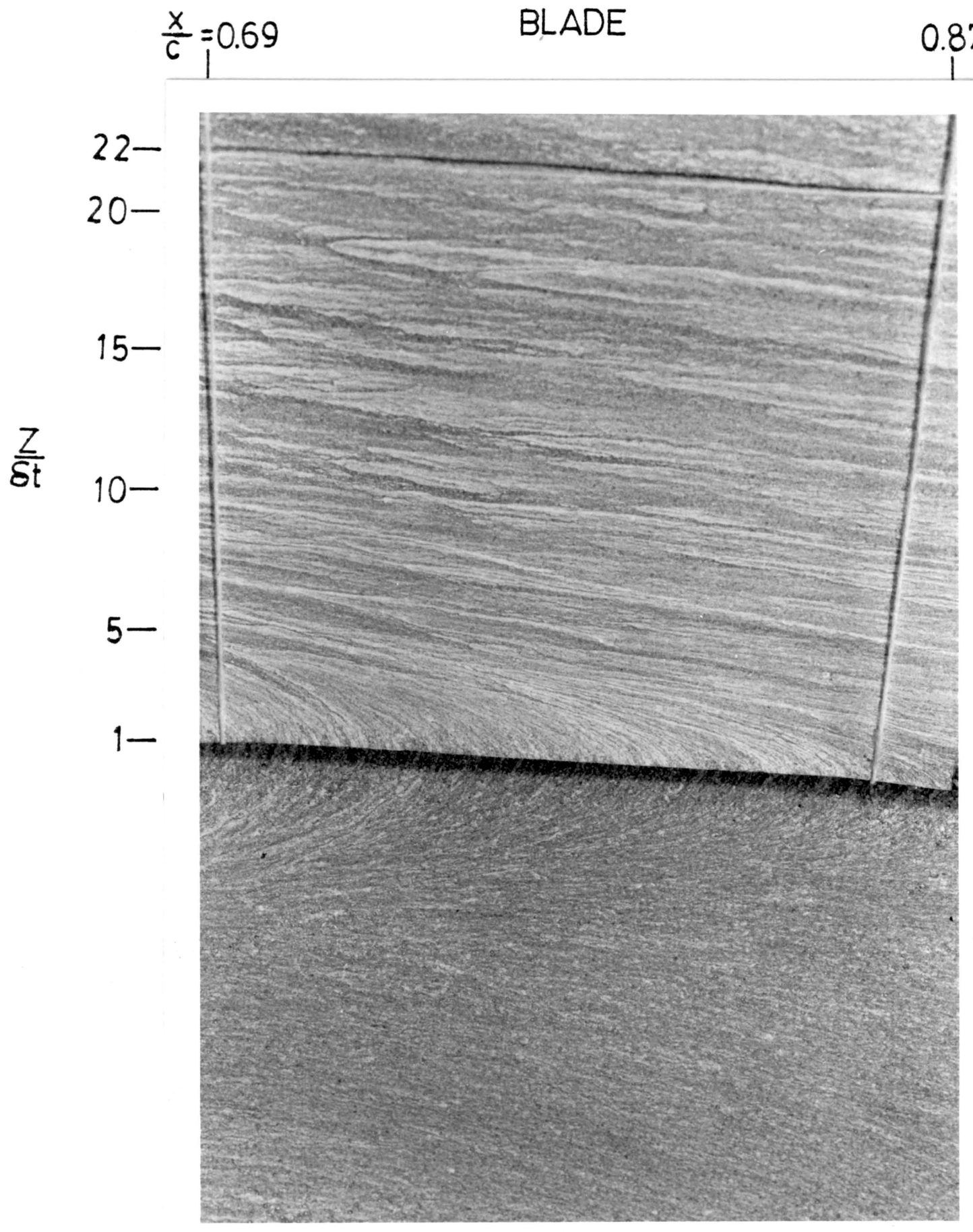

\section{ENDWALL}

Figure 13. Pressure Surface Flow Visualization 


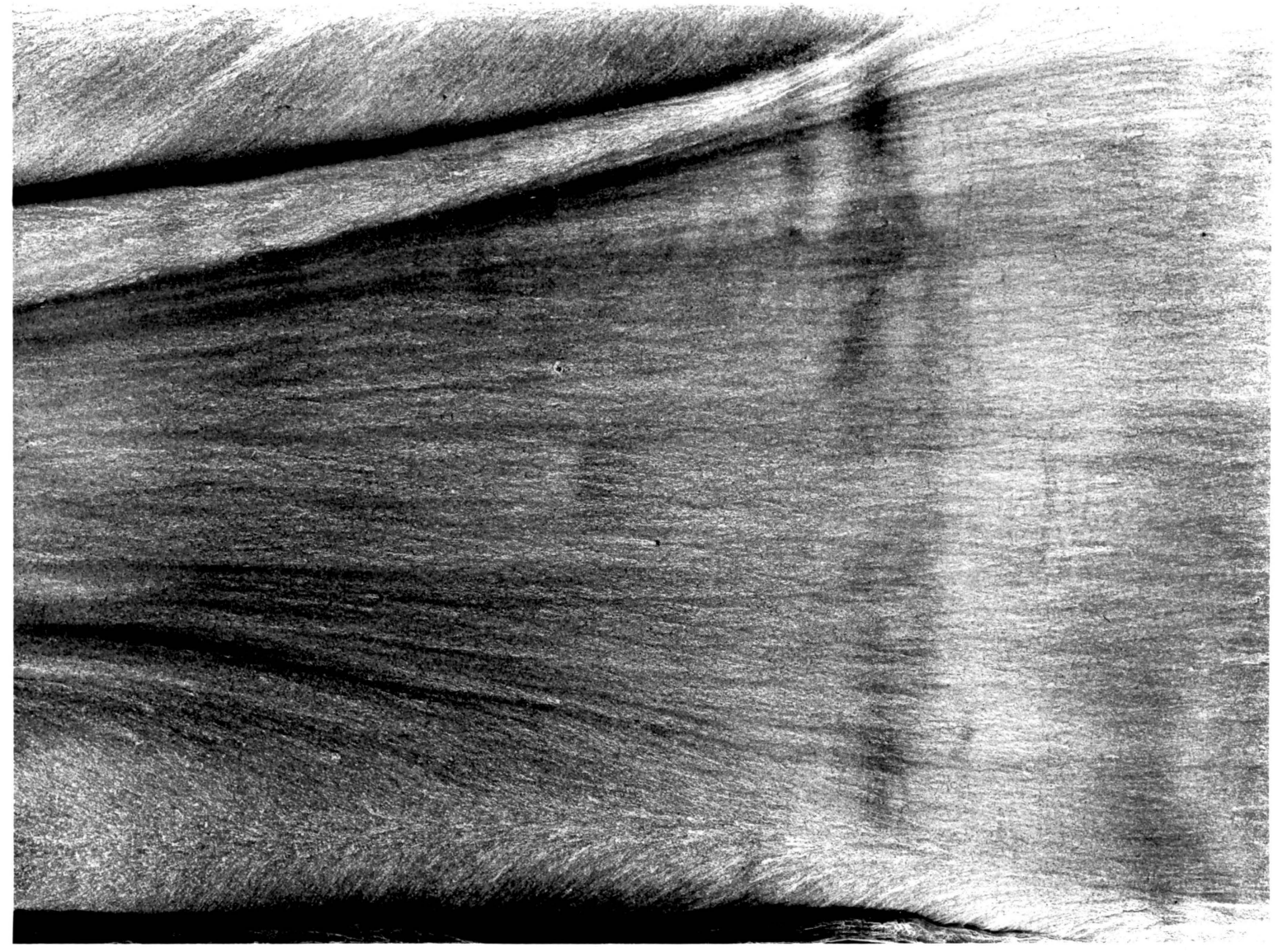

Figure 14. Suction Surface Flow Visualization: Moore and Tilton [ 1 ] 
these three dimensional flows. This apparently almost two dimensional profile boundary layer will be discussed further in section 6.5.4.

Figure 15 is the recent visualization showing the suction surface from $x / c$ $=0.69$ to $\mathrm{x} / \mathrm{c}=0.96$ relative to the bottom endwall.

The visualization extends to $45 \%$ of the blade span and the flow direction is from right to left. Traces of the leakage jet can be seen emerging from the tip gap and then separating from the endwall at $\mathrm{S} 1$ as the leakage fluid rolls up into a vortex. Between 4 and 7 tip gap heights above the endwall, the leakage vortex is seen to be in contact with the suction surface. Below 4 tip gap heights, the visualization mixture is almost completely removed from the blade surface leaving the area visibly darker. This is possibly due to the combined effects of the leakage jet and the vortex fluid as they pull away from the blade surface.

In the previous two figures, a line of flow attachment, A2, followed by divergence is seen at about 7 tip gap heights above the endwall. The significance of this line is not clear from the visualizations. It could be the result of a displaced passage vortex sitting on top of the leakage vortex. The counter rotating vortices would then form a pattern such as that seen in the visualizations. A second possibility is that the passage vortex never forms as a distinct entity in the lower half of the passage, its high loss fluid being completely engulfed by the leakage vortex. The line could then be explained as a result of the leakage fluid diverging when the vortex contacts the blade surface. 


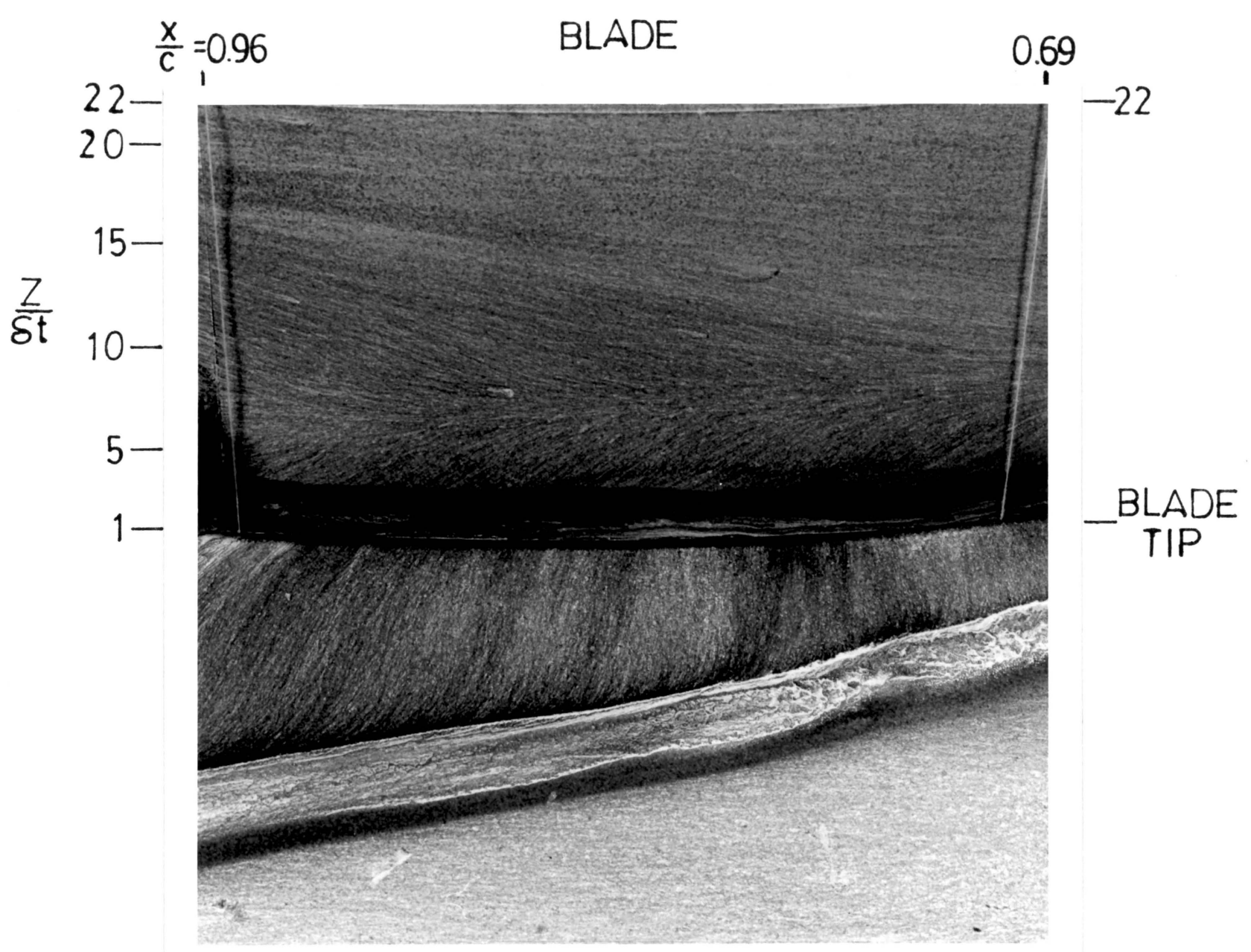

\section{ENDWALL}




\subsection{TUFTED PROBE}

A probe with a wool tuft at its tip was inserted into the flow in an attempt to gain a better understanding of the flow within the blade passage. The leakage vortex as well as the passage vortex in the top half of the passage were easily distinguished. Evidence of the passage vortex was first seen at $x / c \sim 0.45$ where the blade's curvature begins, and it's development could be followed well downstream of the blade trailing edge. The line of attachment and divergence, A2, was also identified, but the fluid flow above that line could not simply be interpreted as due to a passage vortex. Rotation was detected above $\mathrm{A} 2$, but it was not as rapid as that below the line. This is consistent with the evidence in the suction surface visualization showing the flow angles formed with the primary flow direction to be larger below A2.

\subsection{SMOKE VISUALIZATION}

To look at the leakage jet and vortex development within the blade passage, a smoke visualization technique was used. By heating diesel oil, smoke was generated and then injected into the tip gap. The injection was made parallel to the tip gap flow direction in order to minimize disturbances. The high flow velocities within the tip gap could not be attained with the smoke generation equipment; only half the flow rate required for isokinetic injection could be achieved. 
To illuminate the smoke particles, a laser light sheet technique was used. The beam of a $5 \mathrm{~mW}$ laser was spread into a sheet by projecting it through a cylindrical glass rod. With the light sheet positioned in the blade passage at the exit plane of the injected smoke, a cross-section of the tip leakage vortex was revealed.

Figure 16 is a smoke visualization of the leakage fluid which exits the tip gap at $75 \%$ of the blade's axial chord.

The picture was taken perpendicular to the plane of the laser light sheet. The entire leakage vortex is not illuminated because the smoke diffuses very quickly in the highly turbulent fluid. The laser beam can be seen entering from the right and passing through the glass rod which is affixed to blade 2 .

The smoke visualization is superimposed on the endwall and suction surface visualizations in Figure 17.

This gives a three-dimensional perspective of the tip leakage flow as viewed from downstream of the blade trailing edge looking back upstream into the blade passage. The suction surface of blade 3 is seen on the left with smoke marking the leakage flow as it comes from under the blade. Of particular interest is the separation of the leakage vortex from the bottom endwall. 


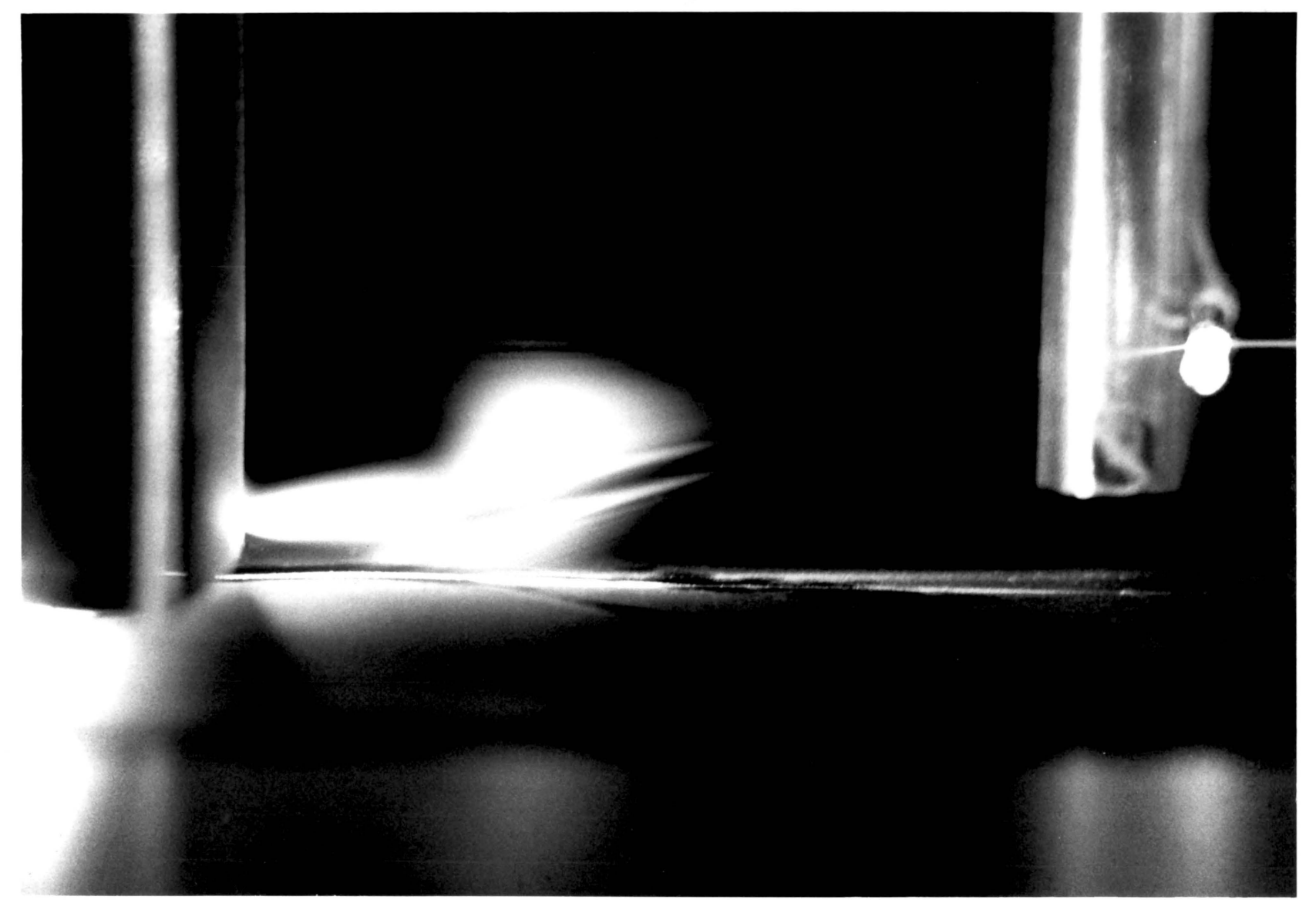

Figure 16. Smoke Visualization of Tip Leakage Flow by Laser Light Sheet 


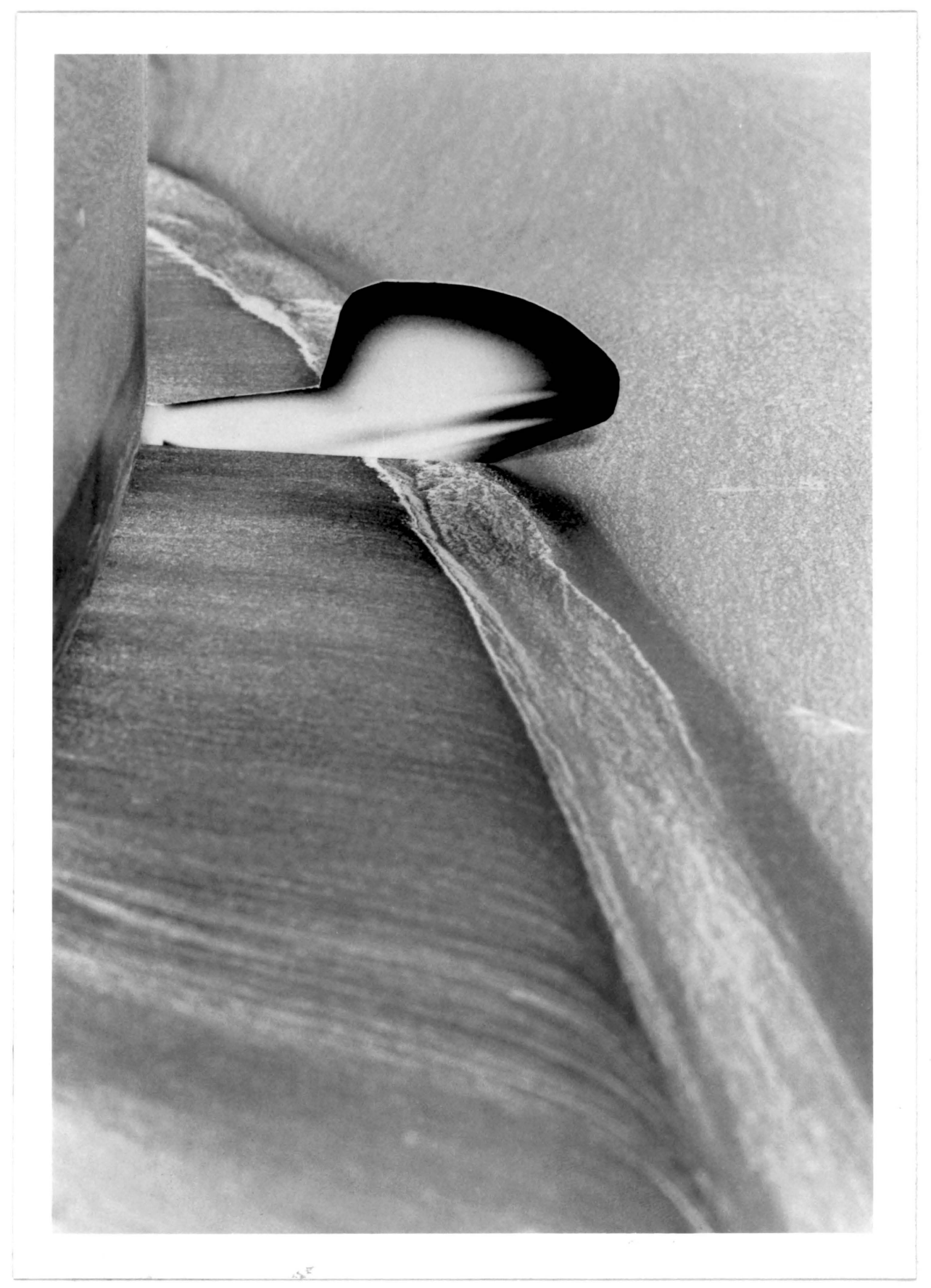

Figure 17. Smoke and Endwall Visualization of Tip Leakage Vortex in Flow Passage: View from downstream with blade suction surface on left 


\section{Chapter 5}

\section{MEASUREMENTS AND PROCEDURE}

Most of the static pressure tappings and instrumentation ports already existing in the cascade were not necessary for the present study. They, therefore, will not be discussed here, except to mention that all the static pressure measurements of Moore and Tilton [ 1 ] were repeated to confirm that the operating parameters of the cascade had not radically changed.

\subsection{TIP GAP EXIT PLANE}

The flow measurements desired for the present study required modifications to the existing cascade. To investigate the flow leaving the tip gap, static pressure tappings and holes for probe insertion were located in the cascade bottom endwall around blade 3, see Figure 18. 


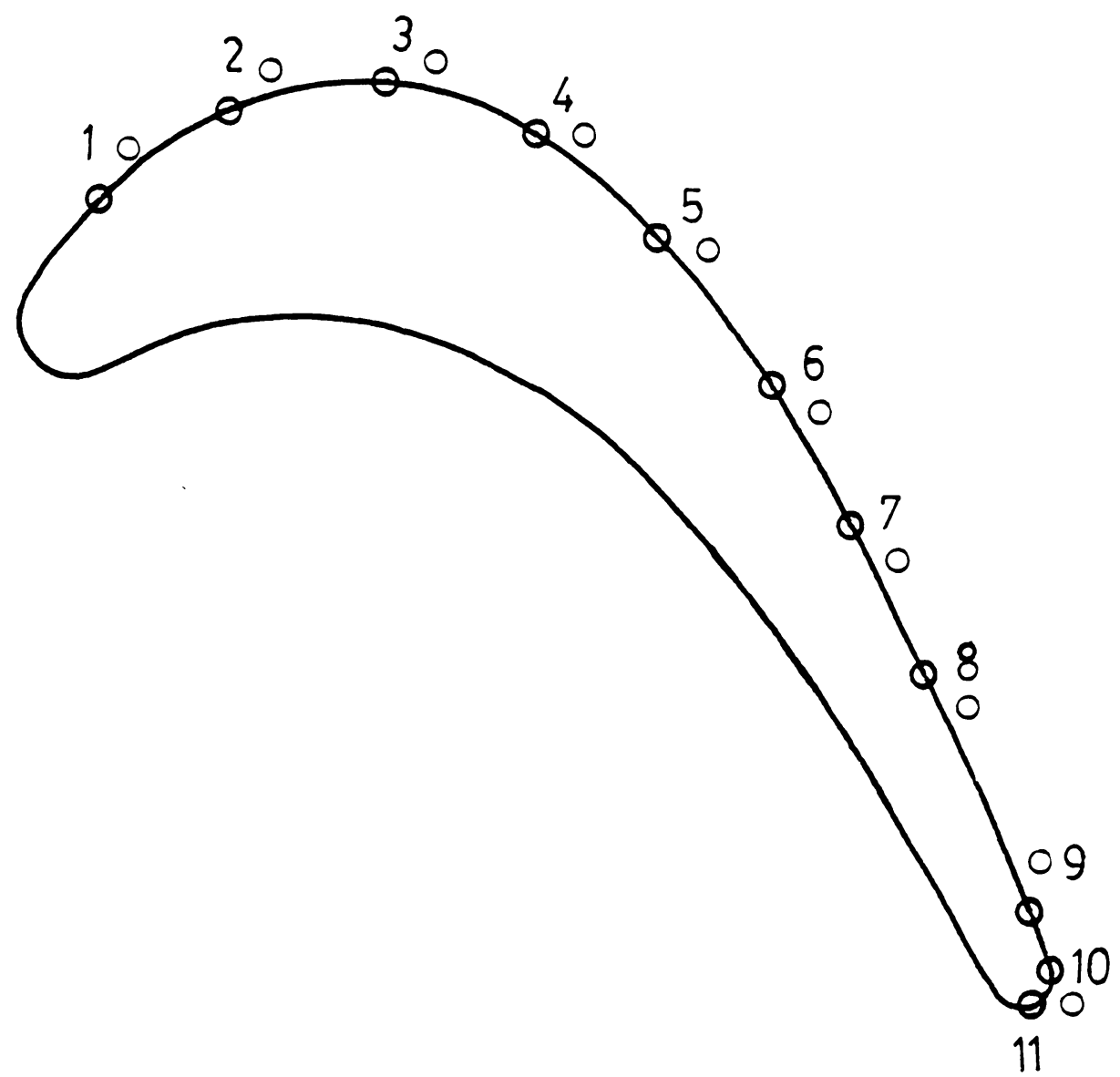

Figure 18. Tip Gap Probe Hole and Static Tapping Locations: Taps on Blade Profile 
Using the endwall surface flow visualization as a guide, ten probe hole locations were chosen. All holes were $4.6 \mathrm{~mm}$ in diameter and were positioned such that when the probe was inserted and nulled in yaw, its tip would be in the tip gap exit plane. Eleven static tappings were used, one corresponding to each probe location and an additional one at the trailing edge. The tappings were set in the tip gap exit plane, just to the side of the probe tip location. In this way interference between the tapping and probe could be avoided. A listing of the probe hole and static tapping locations is given in the following table.

Table 1. Tip Gap Exit Static Tapping and Probe Hole Locations

\begin{tabular}{|c|c|c|}
\hline Measurement Location & \multicolumn{2}{|c|}{ Percent Axial Chord } \\
\hline & Static Tappings & Probe Holes \\
\hline 1 & 7.5 & 12.3 \\
\hline 2 & 20.0 & 23.6 \\
\hline 3 & 34.9 & 39.6 \\
\hline 4 & 48.9 & 54.1 \\
\hline 5 & 60.6 & 65.1 \\
\hline 6 & 71.7 & 76.0 \\
\hline 7 & 79.4 & 83.2 \\
\hline 8 & 86.4 & 90.0 \\
\hline 9 & 96.0 & 96.5 \\
\hline 10 & 99.1 & 102.2 \\
\hline 11 & 96.0 & $-\cdots$ \\
\hline
\end{tabular}

The tip gap exit plane traverses were done vertically beginning with the probe tip on the bottom endwall. The probe tip was raised $0.254 \mathrm{~mm}$ ( $0.01 \mathrm{in}$. ) after each measurement until the blade tip was reached. A total of eighteen measurement were made on each traverse. The height of the tip gap was extremely uniform during these measurements, ranging from $2.08 \%$ to $2.13 \%$ of the blade height. The following 
table presents the locations of the measurement planes and their respective tip gap heights.

Table 2. Tip Gap Measurement Planes and Tip Gap Heights

\begin{tabular}{|c|c|c|}
\hline Measurement Plane & Percent Axial Chord & $\begin{array}{c}\text { Tip Gap Height } \\
\% \text { Blade Height }\end{array}$ \\
\hline 1 & 10.2 & 2.1 \\
\hline 2 & 22.6 & 2.1 \\
\hline 3 & 37.5 & 2.1 \\
\hline 4 & 51.6 & 2.1 \\
\hline 5 & 63.3 & 2.1 \\
\hline 6 & 74.4 & 2.1 \\
\hline 7 & 82.1 & 2.1 \\
\hline 8 & 89.1 & 2.1 \\
\hline 9 & 93.8 & 2.1 \\
\hline 10 & 99.6 & 2.1 \\
\hline
\end{tabular}

At each traverse point, measurements of total pressure and flow angle were made. The static pressure was assumed constant at the endwall value. The flow angle was determined by nulling the probe in yaw and reading the angle of the probe tip relative to the traversing gear from the measure dial attached to the probe stem. Then, by measuring the angle of the traversing gear relative to the cascade, the flow direction at each measurement location was determined.

\subsection{DOWNSTREAM}

It was desired to document the flow from the passage between blades 2 and 3 ( see Figure 5 ) at a distance of 1.4 axial chords downstream of the blade 
leading edges. For access to the passage at this location, a $7.4 \mathrm{~mm}(5 / 16 \mathrm{in}$. ) slot was cut parallel to the plane of the blade trailing edges. The slot extended $10 \mathrm{~cm}$ on either side of the flow passage. The flow passage is defined by the extension of the mean camber lines from the trailing edges of blades 2 and 3, see Figure 19.

Also shown are the top-wall slot and the static tappings on the bottom endwall at the measurement plane. The tip gap variation across the cascade during the downstream measurements is shown in Figure 20.

Because of the slot, the top wall static pressures had to be recorded slightly upstream of the measurement plane. Also, in an attempt to avoid interference from the slot, it was decided to take the top near-wall data upstream as well. The data could then be projected at the measured flow angle to the proper plane.

The measurements in the downstream plane began with the five-hole probe. To establish an initial coarse grid, horizontal traverses were done beginning $6.35 \mathrm{~mm}$ ( $1 / 4 \mathrm{in.}$ ) above the bottom endwall. This was deemed the minimum distance ( two probe tip diameters) at which no probe endwall interactions would occur. On each horizontal traverse, measurements were taken every $12.7 \mathrm{~mm}$ ( $1 / 2$ in. ) beginning and ending $38.1 \mathrm{~mm}(1 / 2 \mathrm{in.})$ to either side of the flow passage. This provided some overlap data and a quantative look at the cascade's repeatability. The horizontal traverses were spaced every $12.7 \mathrm{~mm}(1 / 2 \mathrm{in}$. ) in the bottom half of the passage. In the top half of the passage, where flow variations with vertical position were smaller, the horizontal traverses were spaced by $25.4 \mathrm{~mm}$ ( 1 in. ). Due to physical constraints, the five-hole probe could only be used to within $15.0 \mathrm{~mm}$ ( 0.59 in. ) of the top endwall.

With the coarse grid complete, the areas of significant flow variation were revealed. Additional measurements were taken in the blade wakes and in the passage and leakage vortices to create a fine grid. 


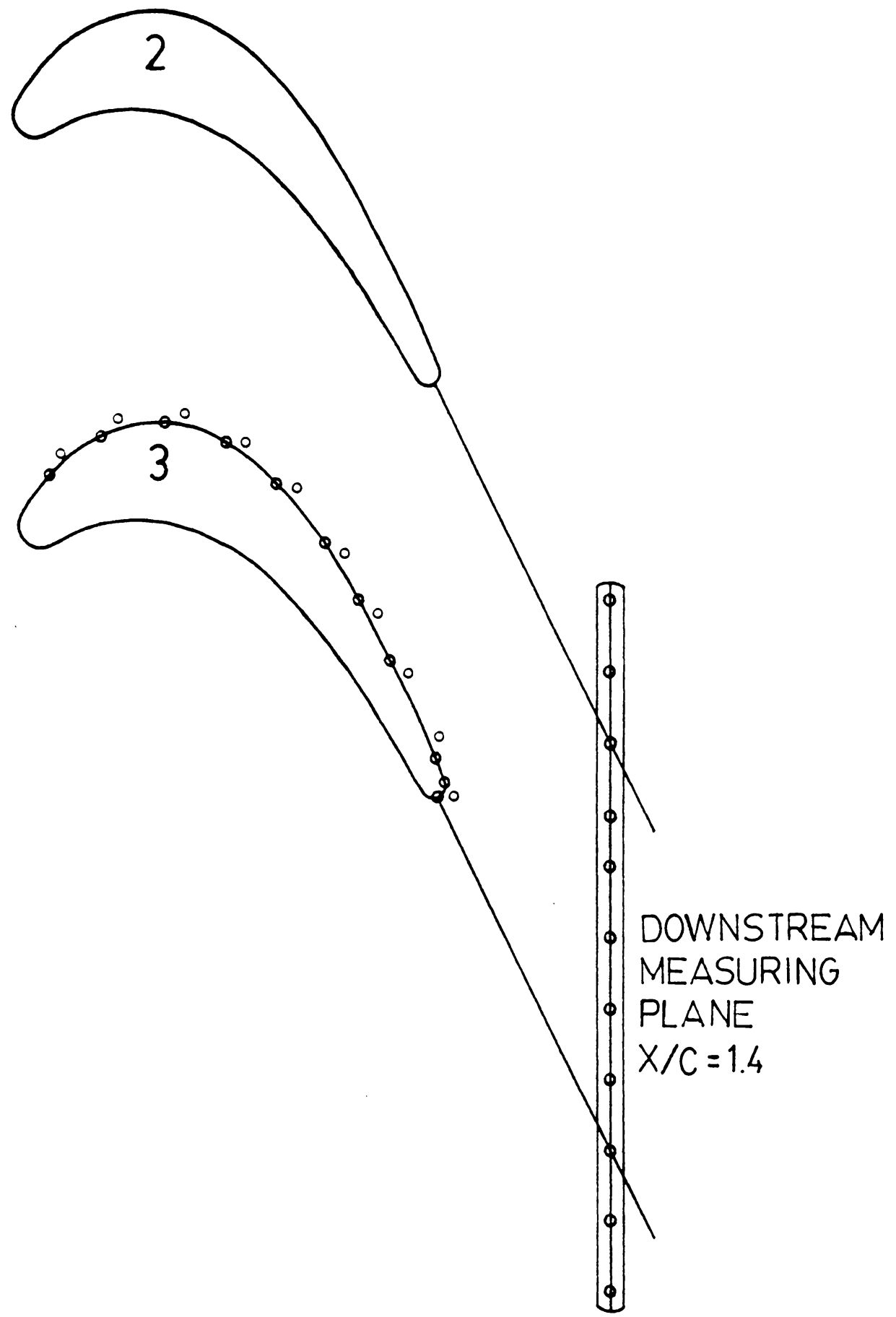

Figure 19. The Flow Passage and Downstream Measuring Plane 


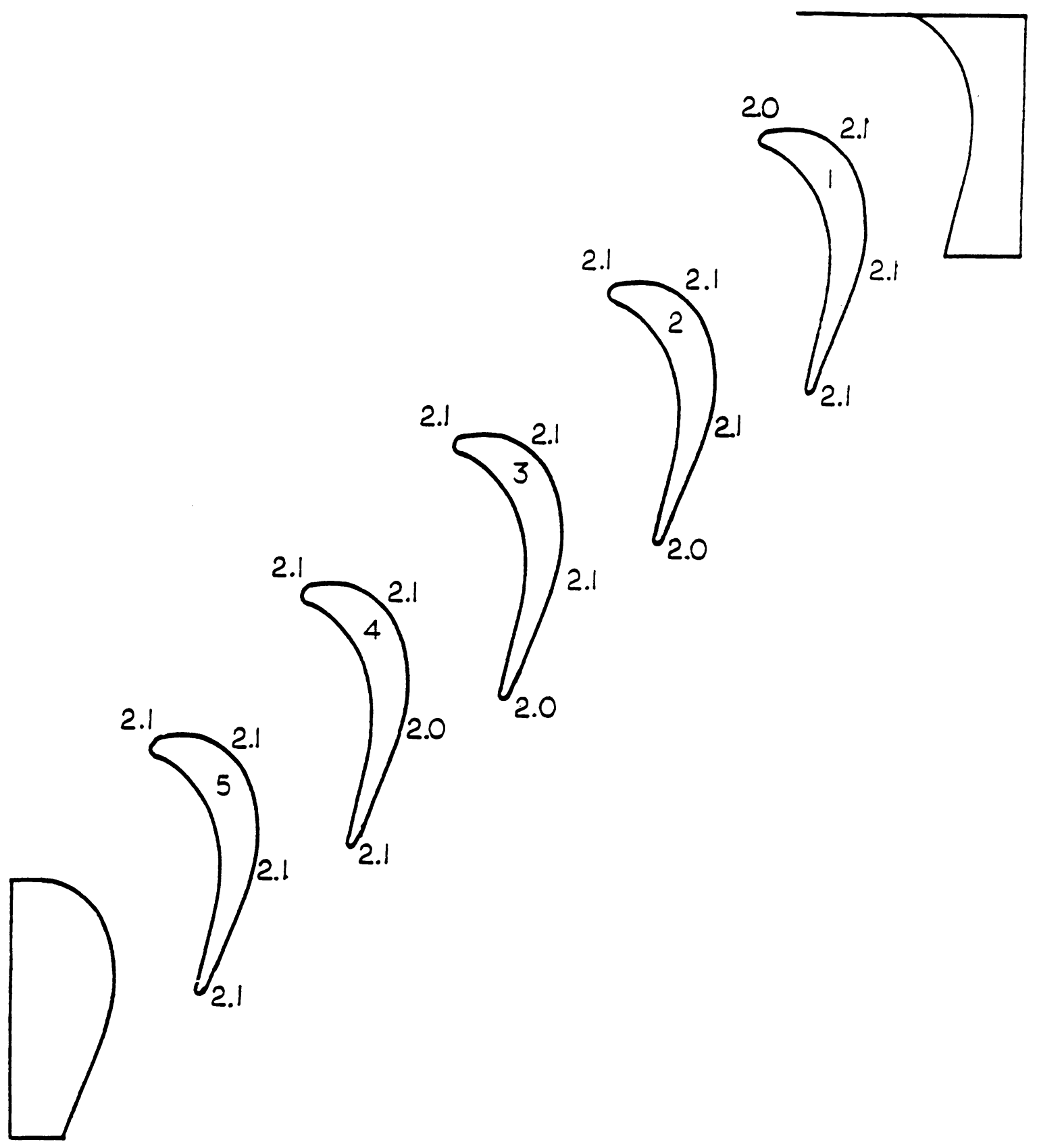

Figure 20. Tip Gap Variation Within Blade Row During Downstream Measurements: Percent of blade height 
To complete the flow picture, the near wall data was taken using the previously described three-hole probes. Initially, traverses were done through the top wall probe holes and at their corresponding locations on the bottom endwall. To reveal the endwall boundary layers, a sufficient number of measurements were taken on each traverse, seventeen at the top wall and twelve at the bottom wall. Because the static pressure variation across the endwalls was very slight at this downstream location, the endwall static pressure at each traverse location was found by linearly interpolating between the nearest static tappings ( see Figure 19 ).

Due to the large velocity gradients in the blade wakes and tip leakage vortex, additional near-wall measurements were necessary. At the bottom wall, the probe was easily positioned to the desired locations through the slot. To avoid drilling extra probe holes in the cascade, the additional top wall measurements were also made from the slot. The probe tip was positioned such that it rested on the endwall just upstream of the slot and the traverses were done. By the agreement of data with that of surrounding traverses, the effects of the slot appeared to be negligible.

For the measurements in the downstream plane, the flow angles were determined as in the tip gap exit plane. Also, to prevent flow leakage, all the instrumentation ports were sealed when not in use. The slot and probe holes were covered with celophane tape on the inside of the cascade and masking tape on the outside. The outside end of the static tappings were also sealed with masking tape. 


\section{Chapter 6}

\section{RESULTS, ANALYSIS AND DISCUSSION}

\subsection{FLOW CONDITIONS}

The laboratory temperature and pressure as well as the cascade inlet total and static pressures were recorded both before and after each set of measurements was taken. The average inlet freestream velocity was $U_{0}=20.5 \mathrm{~m} / \mathrm{s}$. With an average air density of $1.10 \mathrm{~kg} / \mathrm{m}^{3}$ and a viscosity of $0.0000188 \mathrm{~kg} / \mathrm{ms}$, the Reynolds number based on the blade axial chord and exit velocity of $32.7 \mathrm{~m} / \mathrm{s}$ was $4.5 \times 10^{5}$. The inlet endwall boundary layers were assumed to be as measured earlier by Tilton [ 20 ].

$$
\begin{aligned}
\delta_{99} & =2.18 \mathrm{~mm} \\
\delta^{\star} & =0.29 \mathrm{~mm} \\
\theta & =0.19 \mathrm{~mm} \\
H_{12} & =1.52
\end{aligned}
$$


The inlet mass-averaged total pressure loss coefficient was taken to be, $\overline{\bar{C}}_{\mathrm{pt}}=0.0035$. as give by Tilton [ 20 ].

$$
C_{p t}=\frac{P_{t 0}-P_{t}}{\frac{1}{2} \rho U_{0}^{2}}
$$

$$
\text { where: } \begin{aligned}
\mathrm{P}_{\mathrm{t} 0} & =\text { cascade inlet total pressure } \\
& \mathrm{P}_{\mathrm{t}}=\text { local total pressure } \\
\rho & =\text { air density } \\
\mathrm{U}_{0} & =\text { cascade inlet freestream velocity }
\end{aligned}
$$

\subsection{FLOW MEASUREMENTS AT THE TIP GAP EXIT}

\subsubsection{VELOCITY PROFILES}

The tip gap exit velocity profiles were determined from measured values of exit plane static and total pressure. Under the assumption of parallel flow at the tip gap exit plane, the static pressure was considered locally uniform at the endwall value, and the spanwise velocity was assumed to be negligible.

The tip gap exit velocity profiles are shown in Figures 21 and 22.

The profiles were extrapolated to the bottom endwall using $1 / n^{\text {th }}$ power law approximations for turbulent boundary layers. The matching of the fitted curves with the 


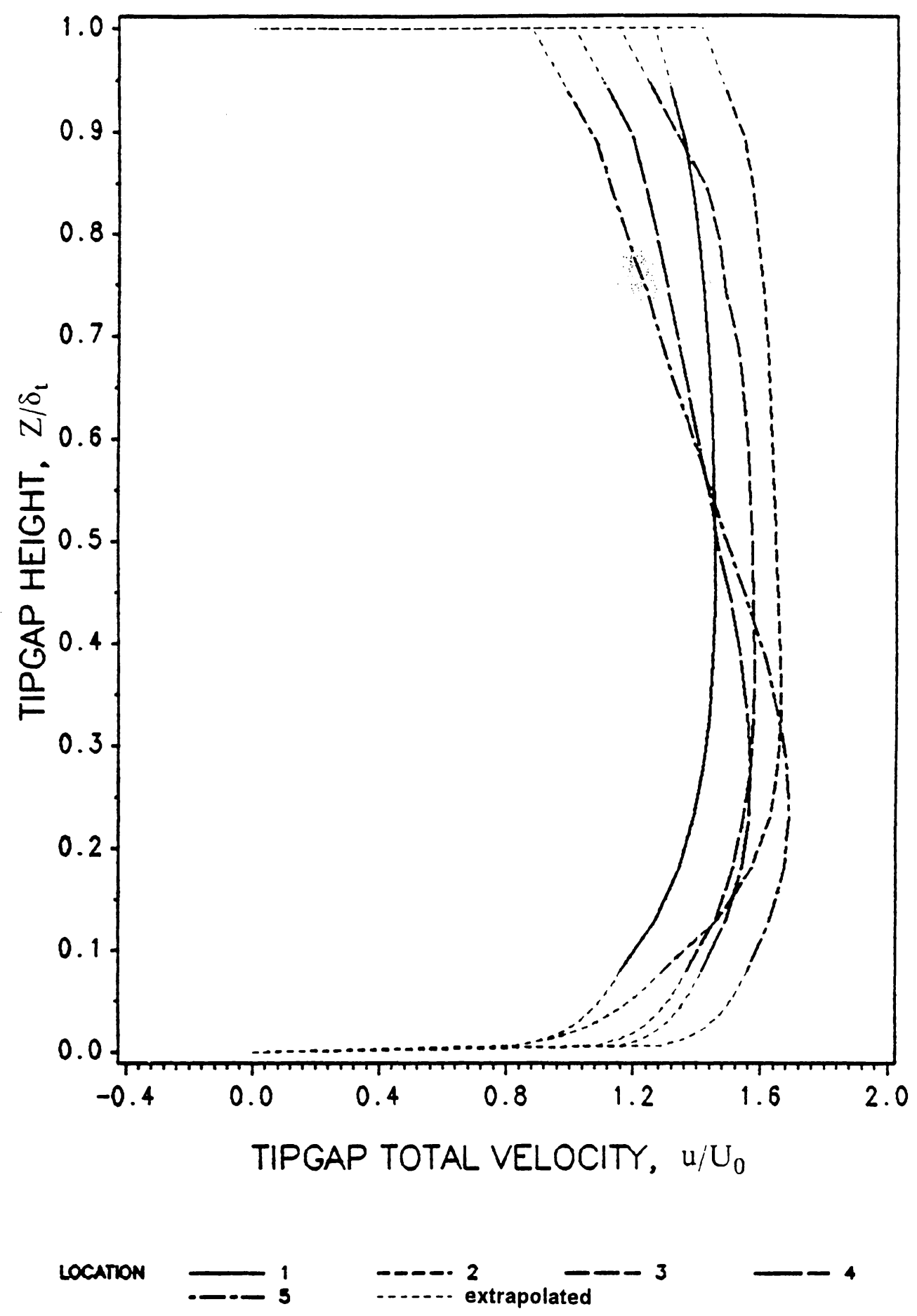

Figure 21. Tip Gap Total Velocity Profiles: Locations 1 through 5 


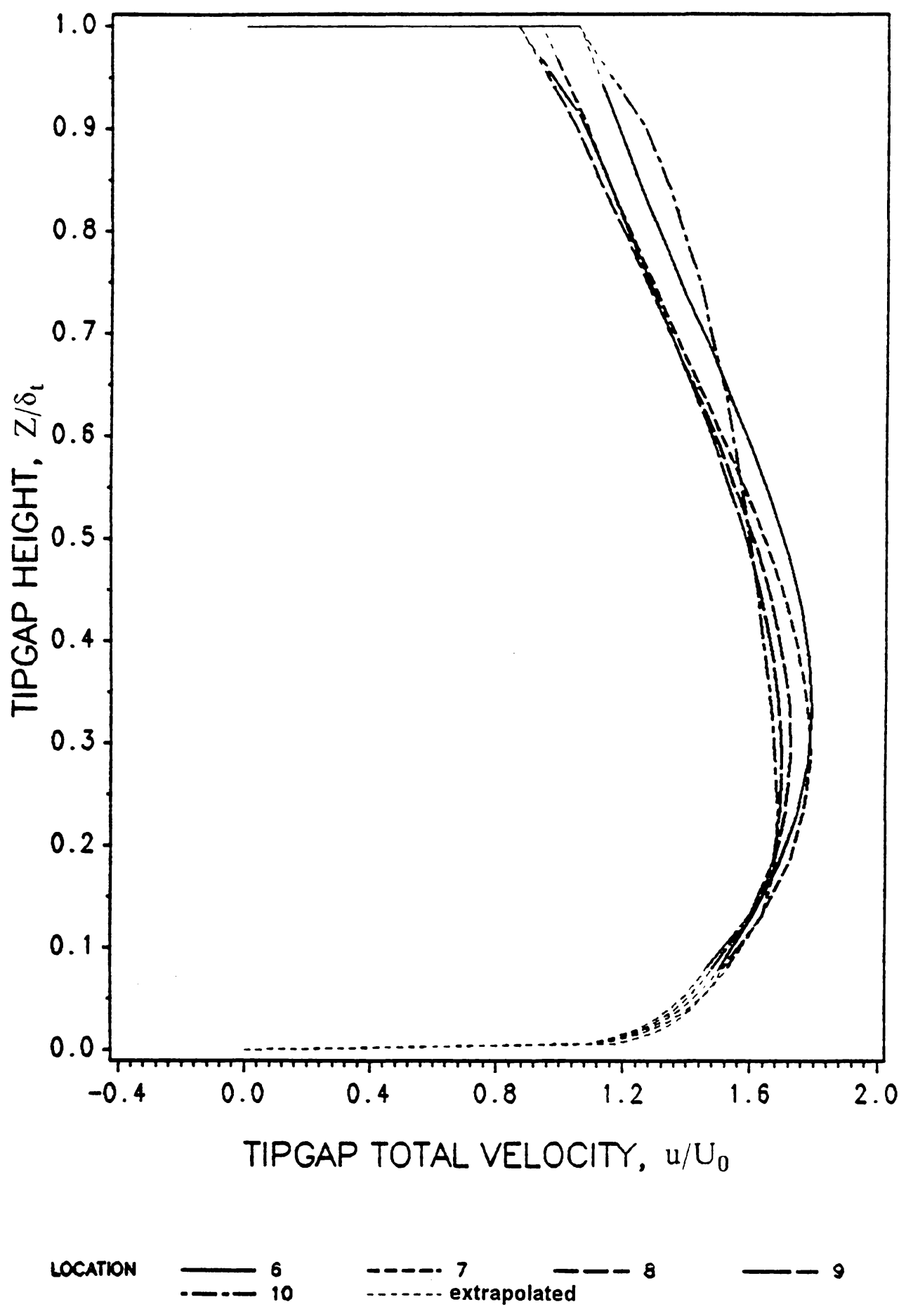

Figure 22. Tip Gap Total Velocity Profiles: Locations 6 through 10 
actual data indicates the choice of a turbulent boundary layer profile was appropriate. This confirms the finding of Moore and Tilton [ 1 ] that the endwall boundary layer was turbulent at the tip gap exit. A particular profile for the blade tip boundary layer could not be determined from the measured data. This is probably due to the extreme thinness of boundary layer which is newly forming after the vena-contracta. Because the area of the boundary layer's influence is so small, the profiles were simply extrapolated linearly to the blade tip.

The velocity profiles correspond to a flow through a vena-contracta followed by flow mixing to fill the tip gap. The extent of the mixing can be judged by the shape of the velocity profile. The uniformity of the profile at location 2 indicates almost complete flow mixing while the curved profiles found toward the blade trailing edge (locations $5 \rightarrow 10$ ) show less evidence of mixing. The peak tip gap exit velocities are of the same order, $\sim 1.6 U_{0}$, as the cascade exit velocity.

\subsubsection{STATIC PRESSURE}

The static pressures at the tip gap exit plane are expressed as pressure coefficients in Figure 23.

$$
C_{p s}=\frac{P-P_{s 0}}{\frac{1}{2} \rho U_{0}^{2}}
$$

where: $P=$ local static pressure

$$
P_{\mathrm{so}}=\text { cascade inlet static pressure }
$$




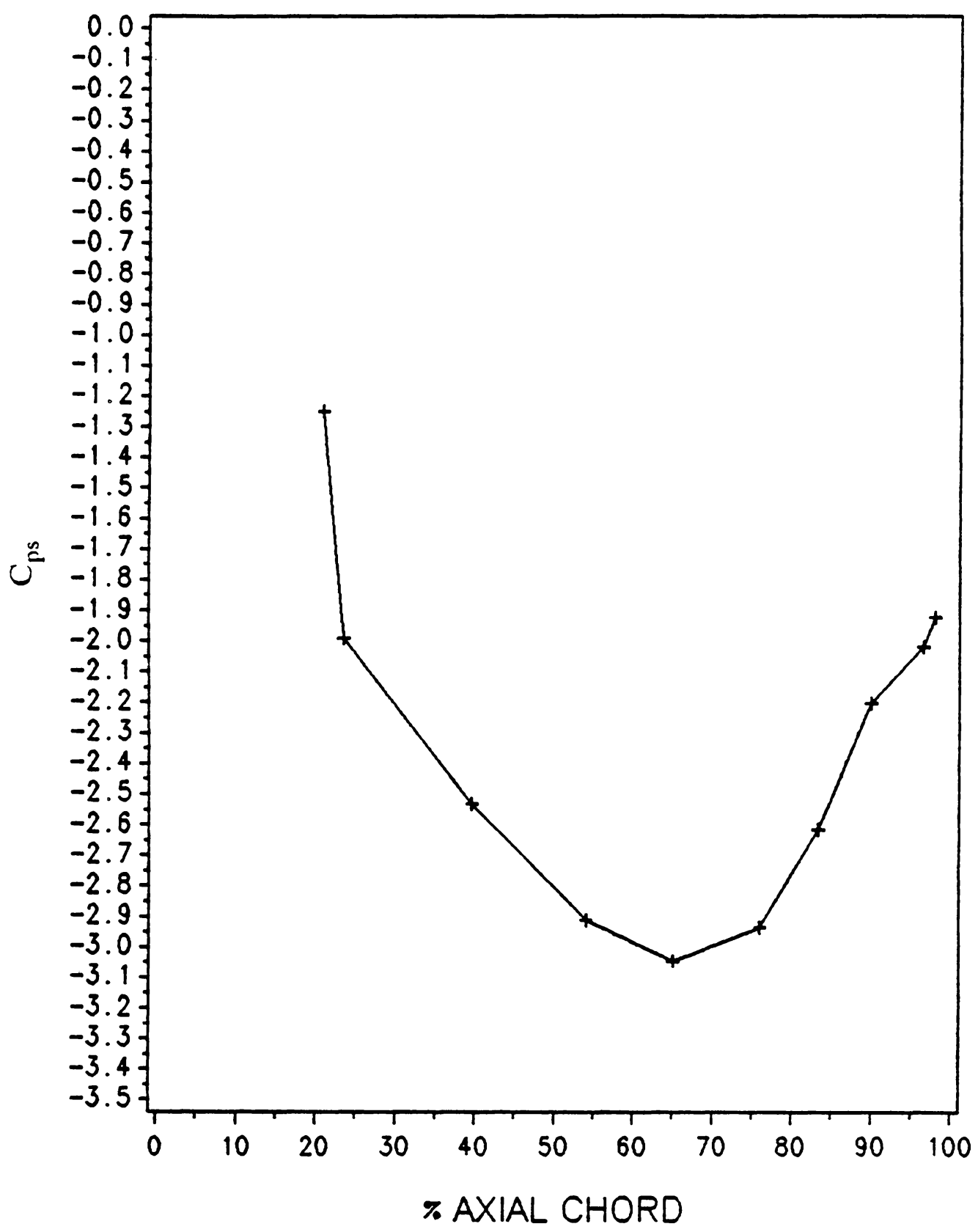

Figure 23. Tip Gap Exit Static Pressure Coefficients 
The lowest static pressures are seen to exist near mid-axial chord. Since the static pressure difference across the blade tip is the driving force of the leakage flow, the strength of the leakage jet is expected to be greatest in this area. This is confirmed by the velocity profiles of Figures 21 and 22 . This increase in strength corresponds to the increased penetration of the tip leakage flow into the passage which was seen earlier in the endwall flow visualization, Figures 11 and 12.

\subsubsection{TOTAL PRESSURE}

The distribution of total pressure loss coefficient ( eq. 6.1) at the tip gap exit plane for each measurement location is shown in Figures 24 and 25.

The near wall values were calculated from the extrapolated values of velocity. Again, the degree of mixing can be seen by the profile uniformity. The highest losses are seen near the blade tip where the flow is mixing to fill the tip gap. In the bottom half of the tip gap the losses are generally much lower. In particular, the profile at location 9 shows little evidence of mixing and seems to still possess a potential flow core at about 0.2 tip gap heights.

\subsubsection{DIRECTIONAL VELOCITIES}

By measuring the direction normal to the blade surface at each probe location and comparing it to the measured flow direction, the tip gap velocities were broken up into normal and streamwise components. The streamwise direction is defined as tangent to the blade surface and positive in the direction of the primary flow. Alpha 


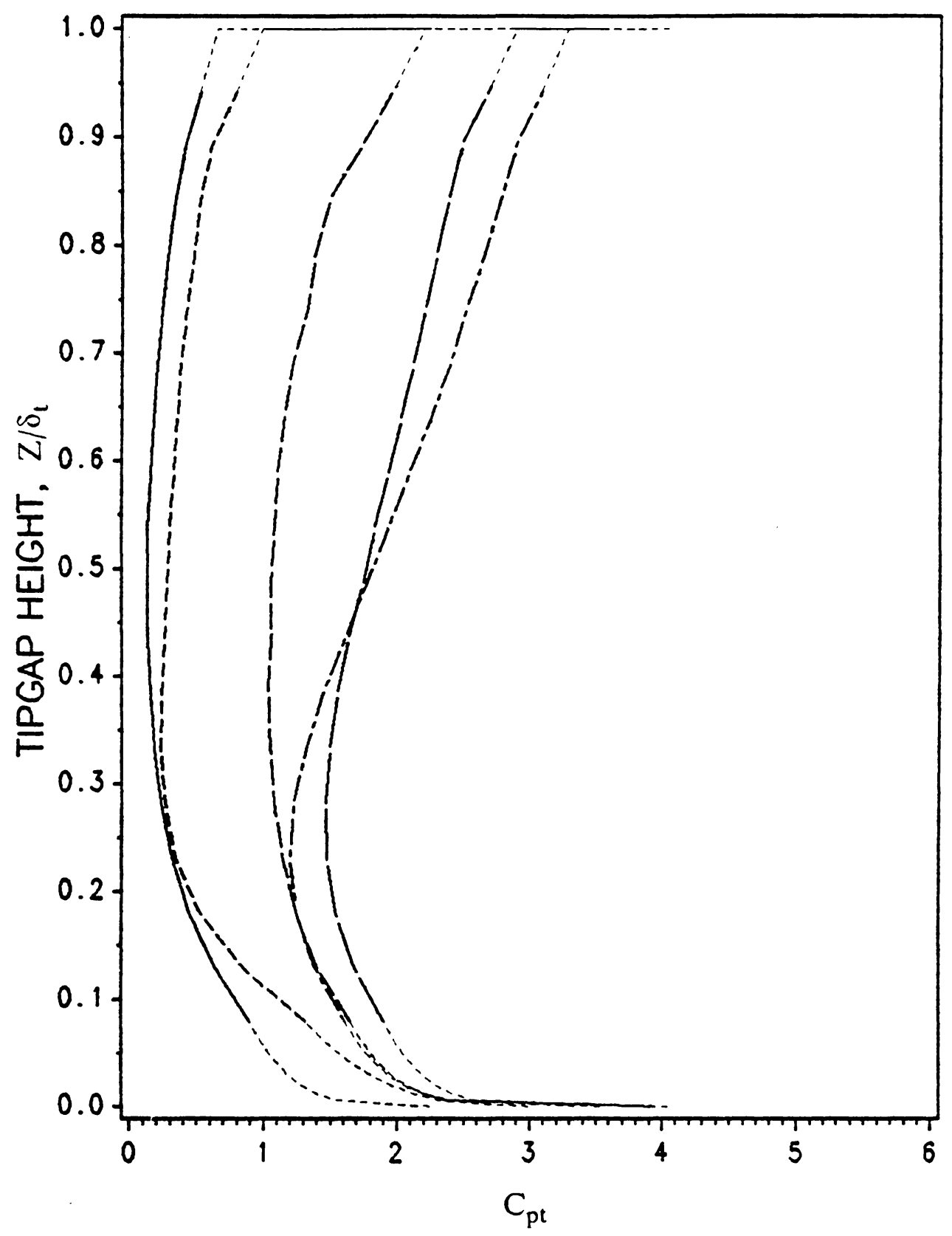

LOCATION $=-15 \quad--\cdots \cdot 2$ extrapolated $-3 \quad-3$

Figure 24. Total Pressure Loss Coefficients at the Tip Gap Exit: Locations 1 through 5 


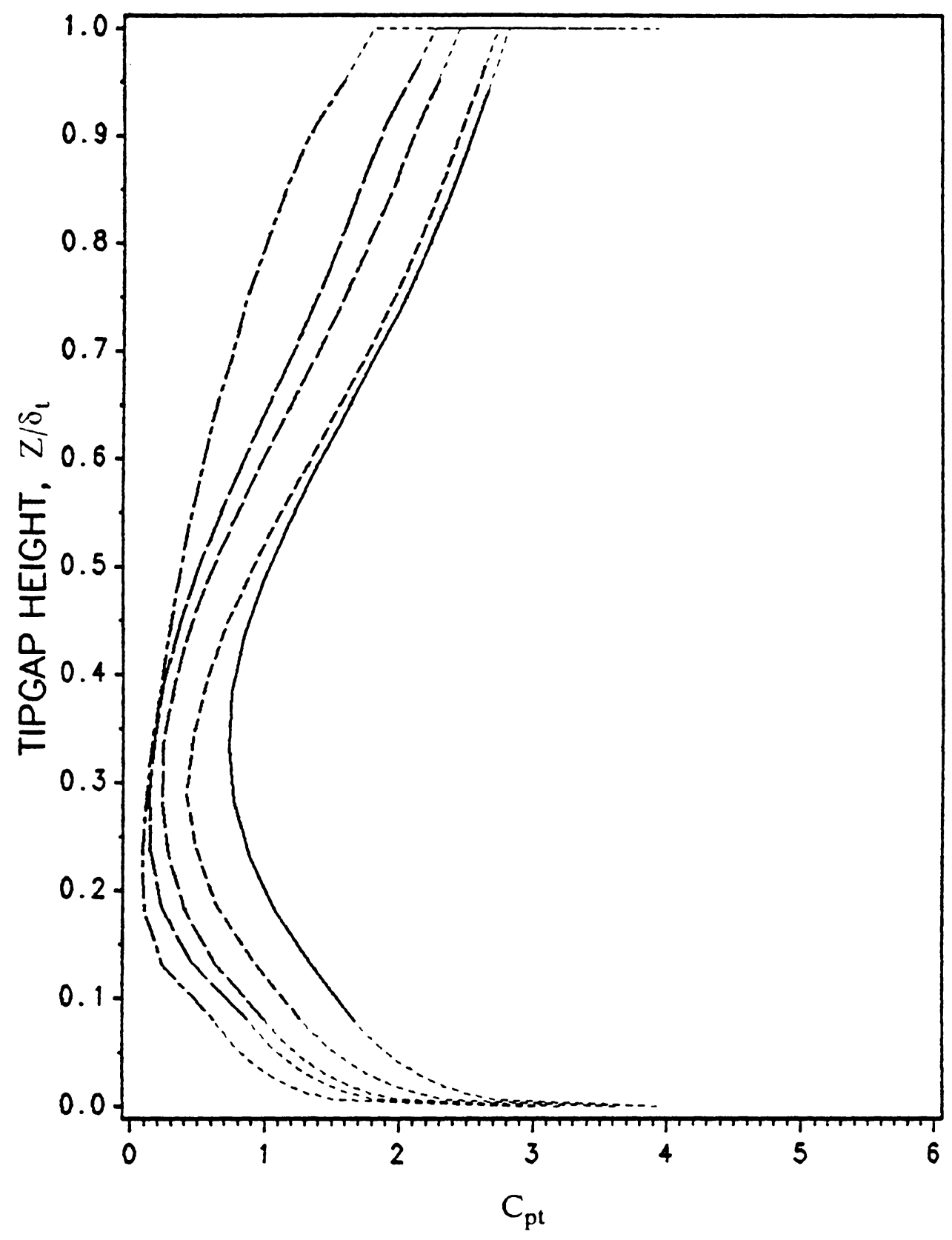

LOCATION

Figure 25. Total Pressure Loss Coefficients at the Tip Gap Exit: Locations 6 through 10 
$(\alpha)$ is the flow angle with respect to the blade normal; it is positive toward the streamwise direction, see Figure 26.

The distribution of flow angle, $\alpha$, at each of the measurement locations is given in Figures 27 and 28.

The flow angles are seen to remain relatively constant across the tip gap height. This observation and a comparison with the endwall flow visualization led to the assumption of a uniform flow angle between the endwall and the nearest measurement point. As with the velocities, the flow angles were extrapolated linearly to the blade tip. The normal and streamwise velocities calculated from the above mentioned flow angles are presented in Figures 29 through 32.

A qualitative understanding of the tip leakage flow can be gained by inspection of Figures 27 through 32. Near the blade leading edge (location 1 ), $\alpha$ is seen to be very high $\left(65^{\circ} \rightarrow 75^{\circ}\right)$ and the flow is primarily in the streamwise direction. The normal component of the velocity is very small meaning that little flow is discharging from the tip gap. Moving around the blade towards the trailing edge, the flow direction becomes more and more normal to the blade as the leakage flow becomes stronger. By $54 \%$ axial chord ( location 4 ) the flow is essentially normal to the blade. The flow direction continues to change and at $65 \%$ axial chord (location 5 ) the flow actually leaves the tip gap with a velocity component in the negative streamwise direction. As the trailing edge is approached, the streamwise component turns positive again and continues to increase. The profiles on the trailing edge circle (location 10 ) should be considered with care because the definitions of normal and streamwise velocity components are no longer valid since the blade suction surface tangent is no longer in the direction of the primary flow.

The above mentioned behavior can be generally explained by the variation of tip gap exit plane static pressure. As seen if Figure 23, the exit static pressure is 


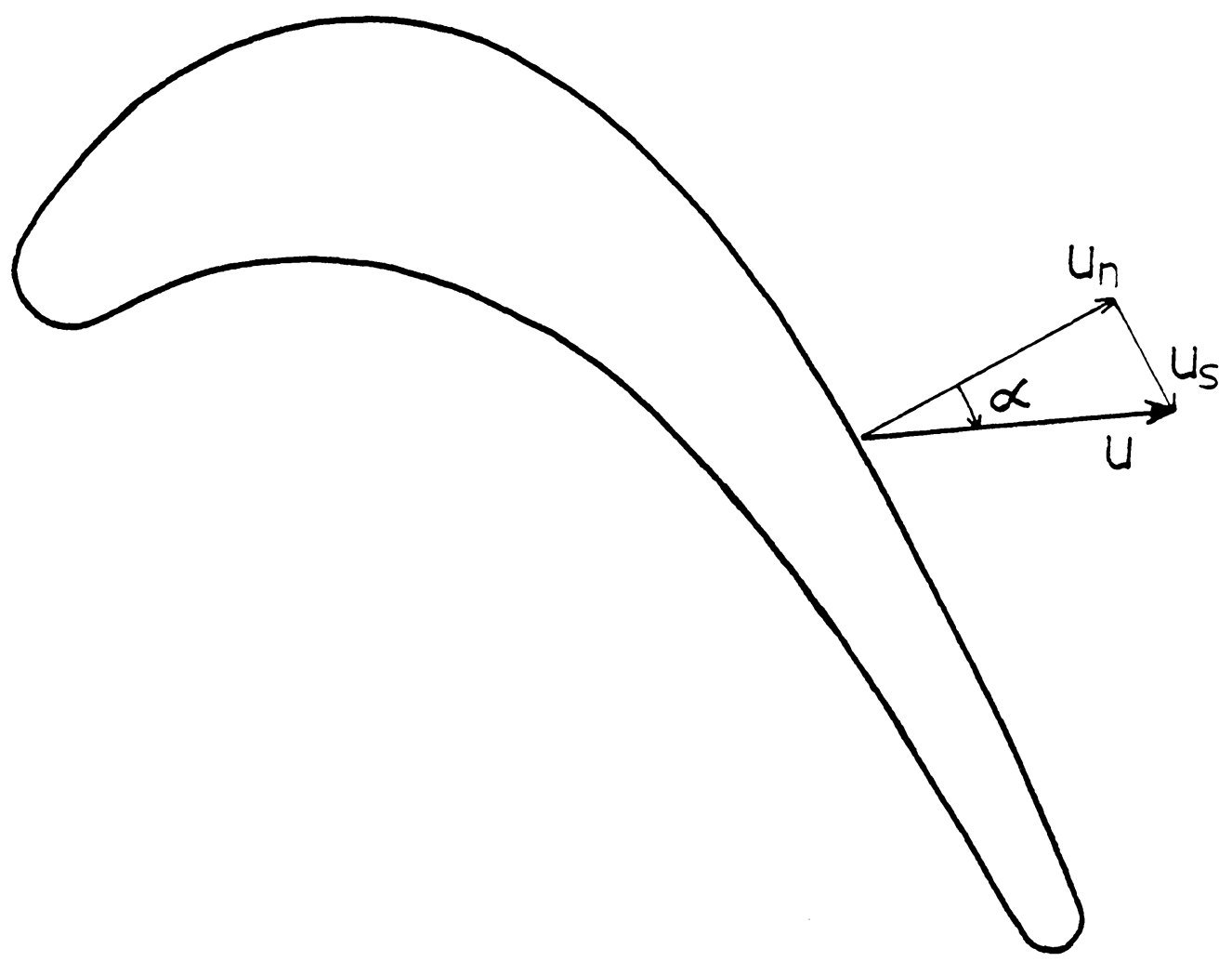

Figure 26. Flow Coordinate System at Tip Gap Exit Plane 


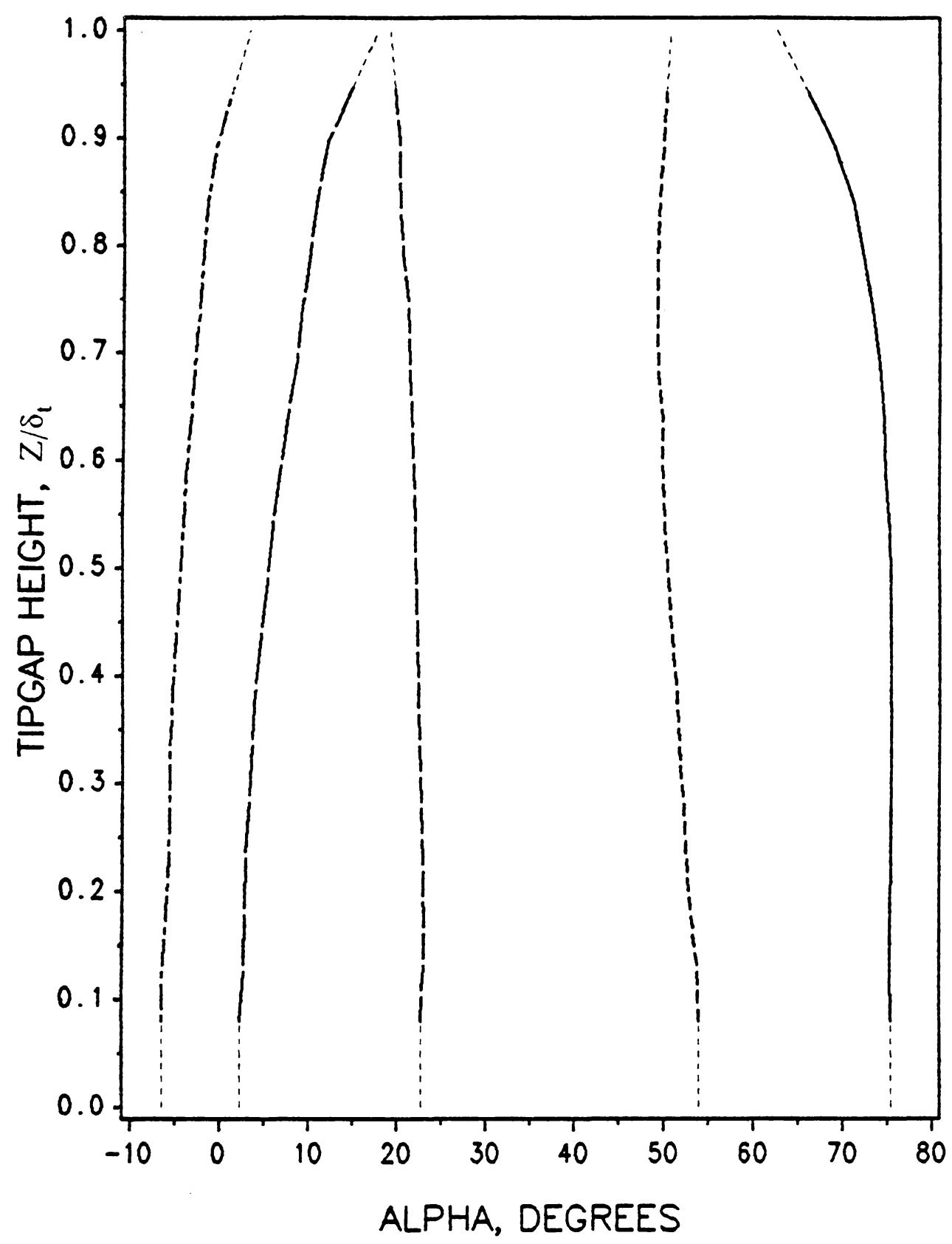

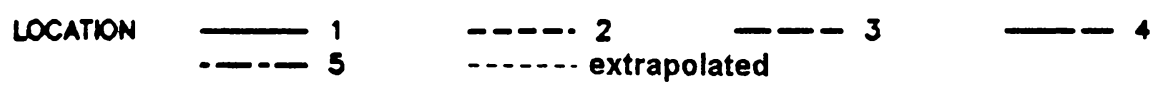

Figure 27. Flow Angle Distribution at the Tip Gap Exit: Locations 1 through 5 


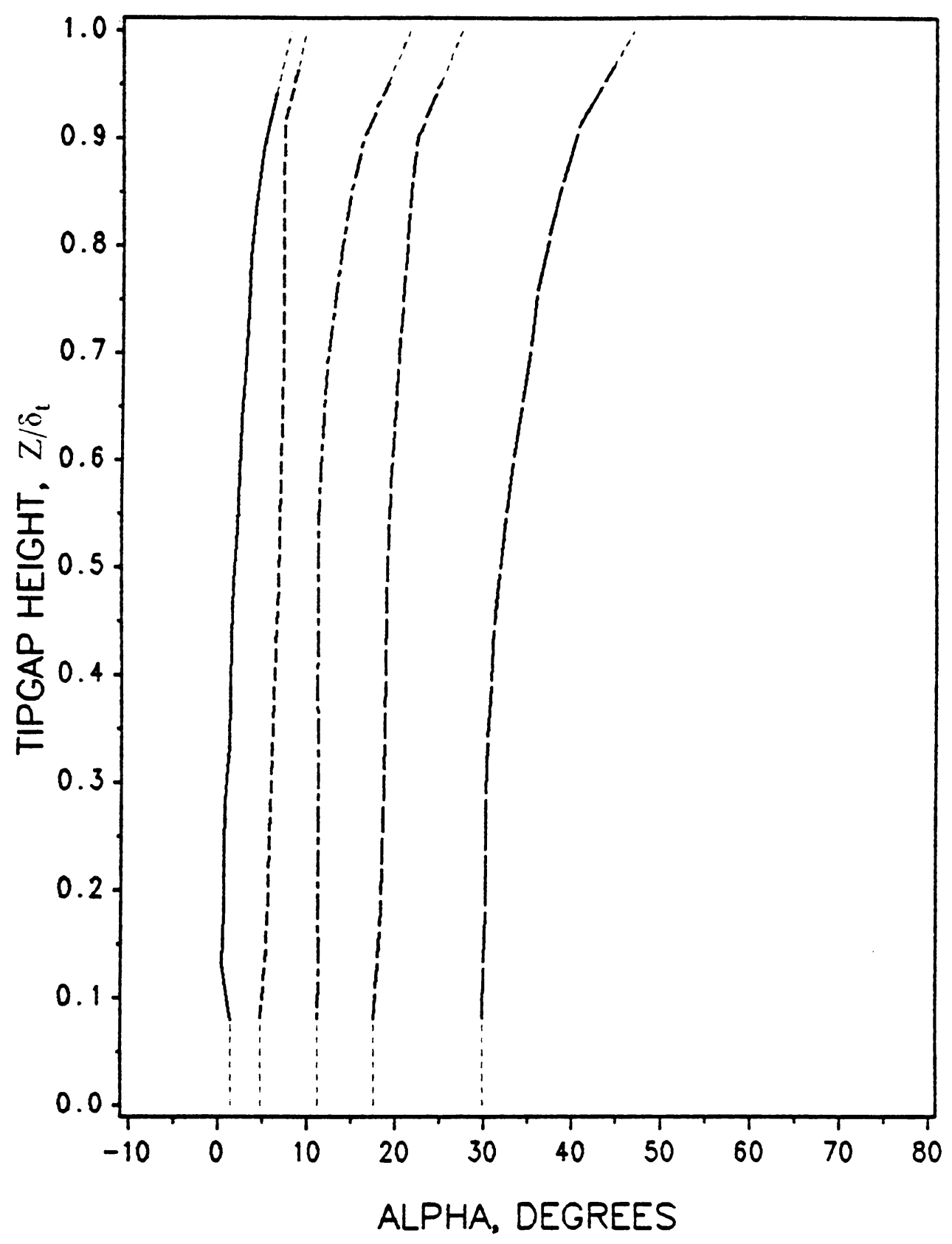

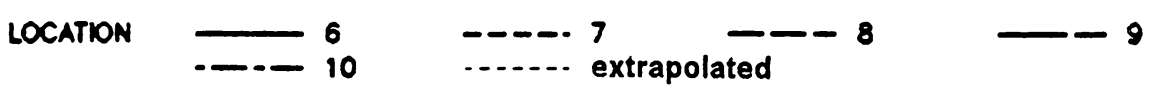

Figure 28. Flow Angle Distribution at the Tip Gap Exit: Locations 6 through 10 

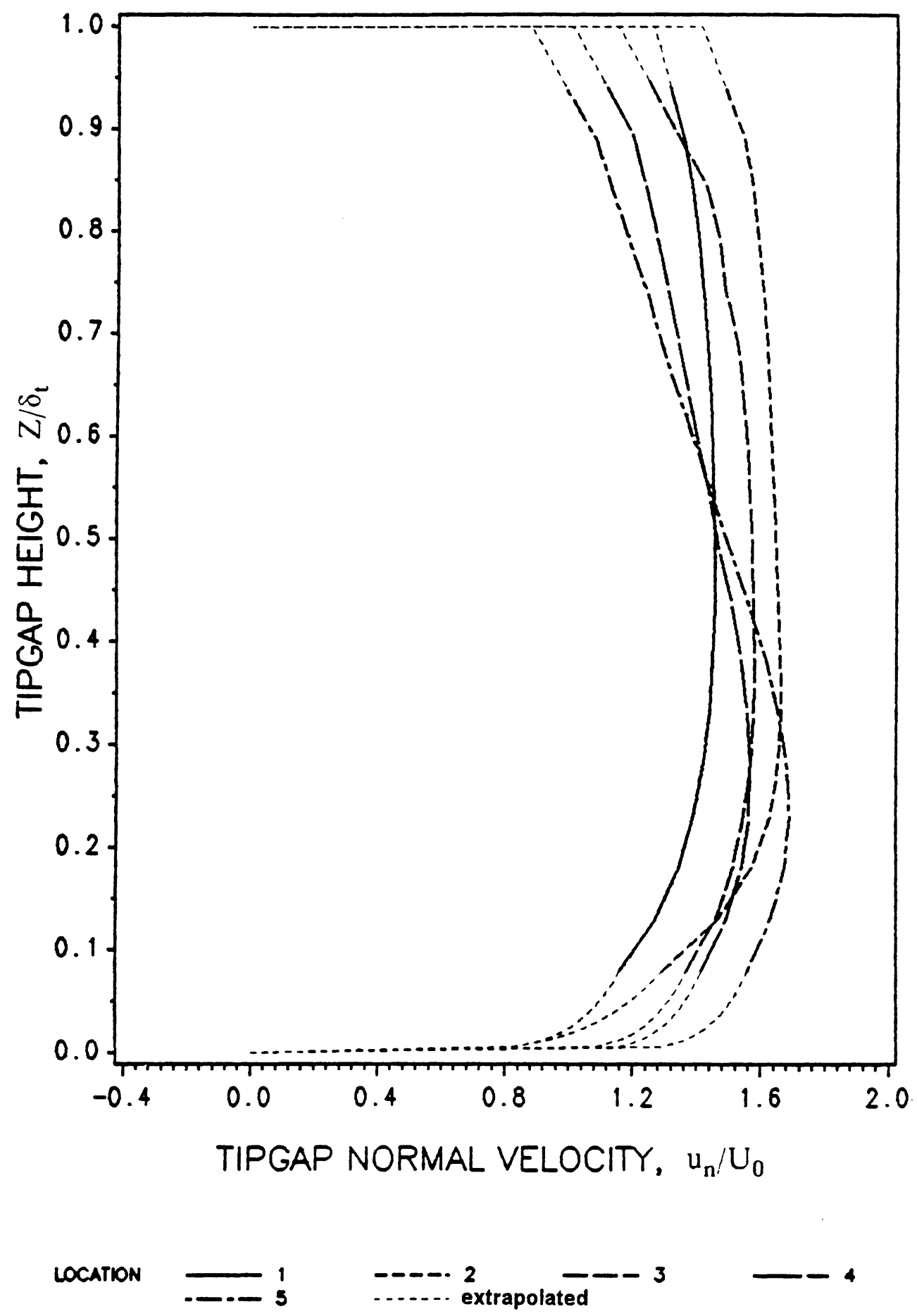

Figure 29. Normal Velocity Profiles: Locations 1 through 5 


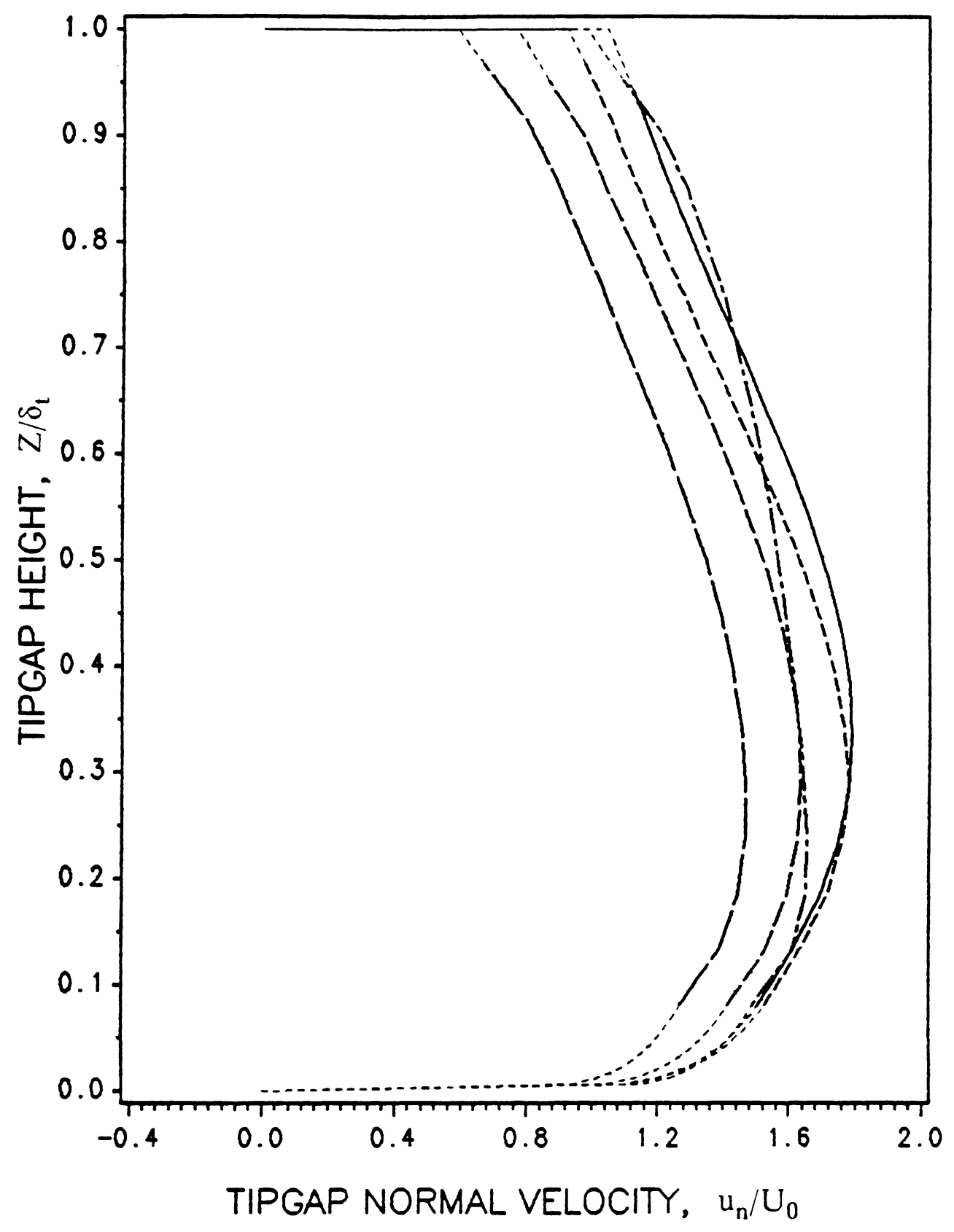

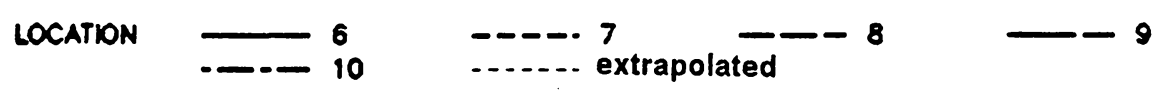

Figure 30. Normal Velocity Profiles: Locations 6 through 10 

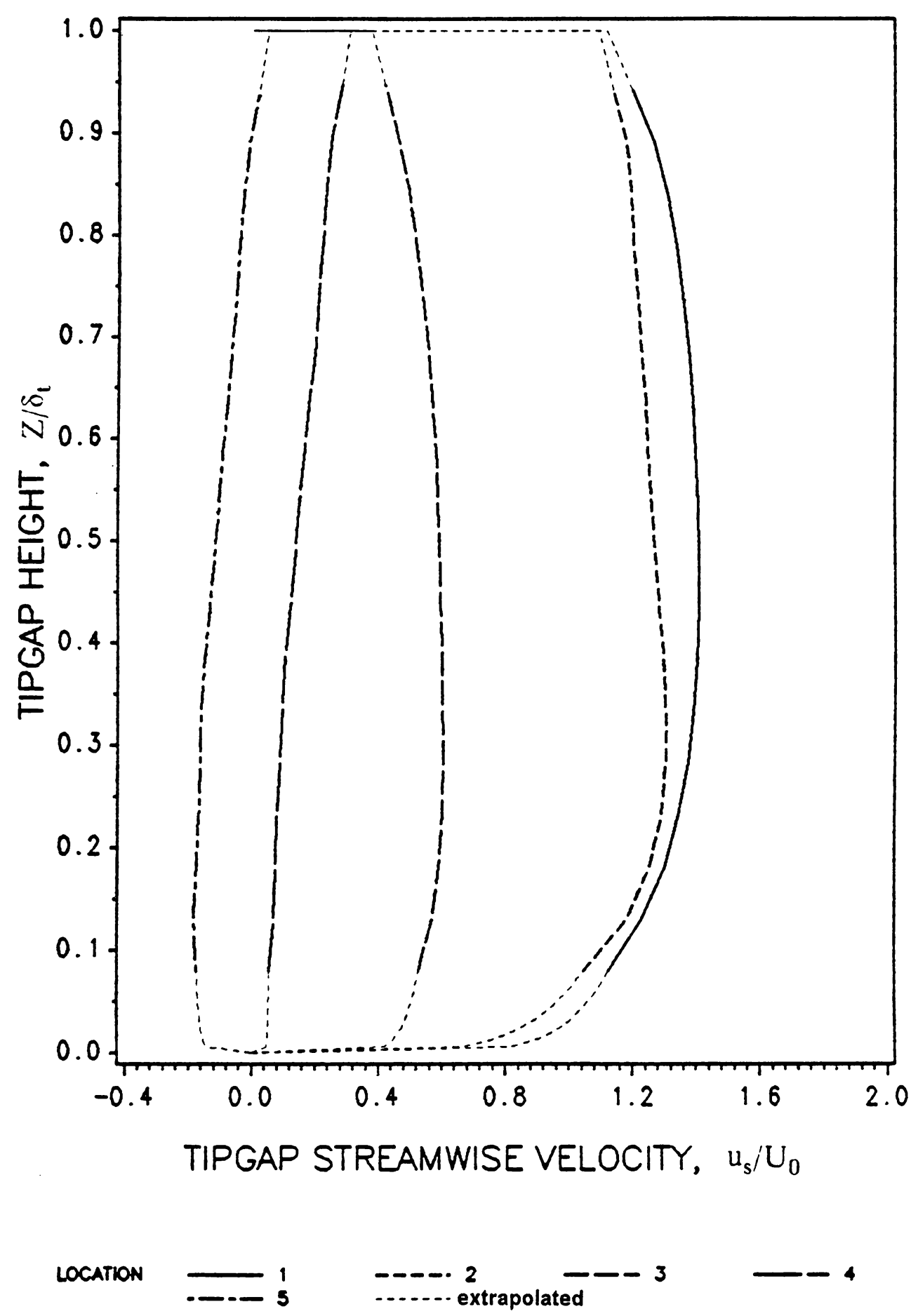

Figure 31. Streamwise Velocity Profiles: Locations 1 through 5 


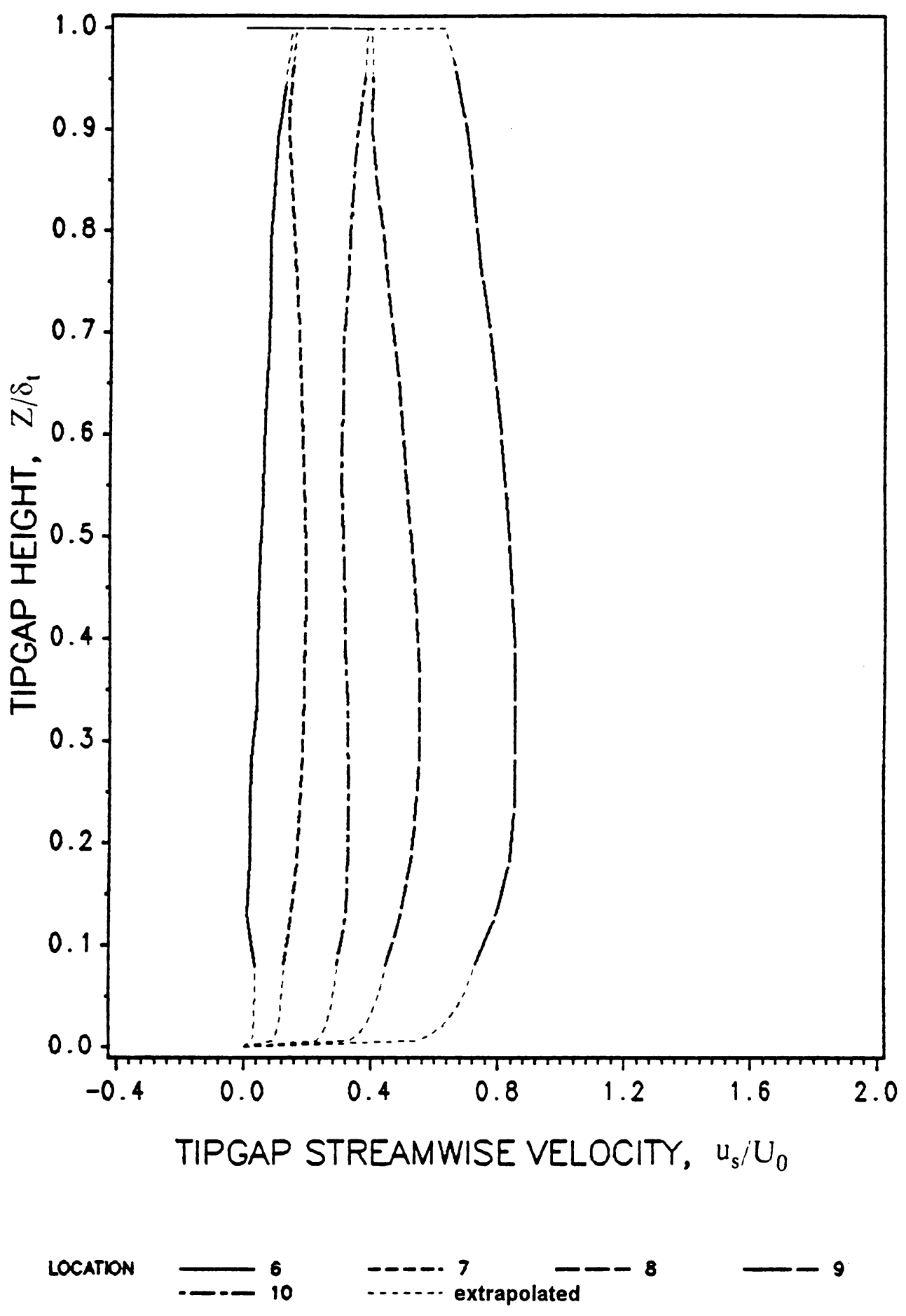

Figure 32. Streamwise Velocity Profiles: Locations 6 through 10 
highest near the blade leading edge providing little driving force for leakage flow. It drops down quickly toward mid-axial chord creating a strong, flow inducing, gradient from the pressure to the suction side of the blade. As the pressure then rises toward the trailing edge, the strength of the leakage flow diminishes and its path turns more in the streamwise direction.

\subsection{INTEGRATED FLOW PROPERTIES}

\subsubsection{DISCHARGE COEFFICIENT}

Following the procedure of Moore and Tilton [ 1 ], a discharge coefficient was calculated for each of the ten tip gap measurement locations.

$$
C_{D}=\frac{\bar{u}_{n}}{U_{t}}
$$

$\bar{u}_{n}$ is the area averaged normal velocity at each measurement location and $U_{t}$ is the tip gap exit velocity from potential flow analysis based on the cascade inlet free stream total pressure $P_{t 0}$ and the local tip gap exit static pressure $P_{\mathbf{s , a x}}$ 


$$
\begin{gathered}
\bar{u}_{n}=\frac{\int_{0}^{\delta_{t}} u_{n} d z}{\delta_{t}} \\
U_{t}=\left[\frac{2}{\rho}\left(P_{t 0}-P_{s, e x}\right)\right]^{1 / 2}=U_{0}\left[C_{p t, 0}-C_{p s, e x i t}\right]^{1 / 2}
\end{gathered}
$$

The calculated values of discharge coefficient around the blade profile are plotted in Figure 33.

Again, the highest values are seen to exist just past mid-axial chord where the flow is most normal to the blade.

\subsubsection{TOTAL PRESSURE LOSS COEFFICIENT}

A mass-averaged total pressure loss coefficient was calculated for each of the measurement locations.

$$
\overline{\bar{C}}_{p t}=\frac{\int_{0}^{\delta_{t}} \rho u_{n} C_{p t} d z}{\int_{0}^{\delta_{t}} \rho u_{n} d z}
$$

where: $\quad C_{p t}=$ total pressure loss coefficient ( equation 6 )

$$
u_{n}=\text { tip gap normal velocity }
$$




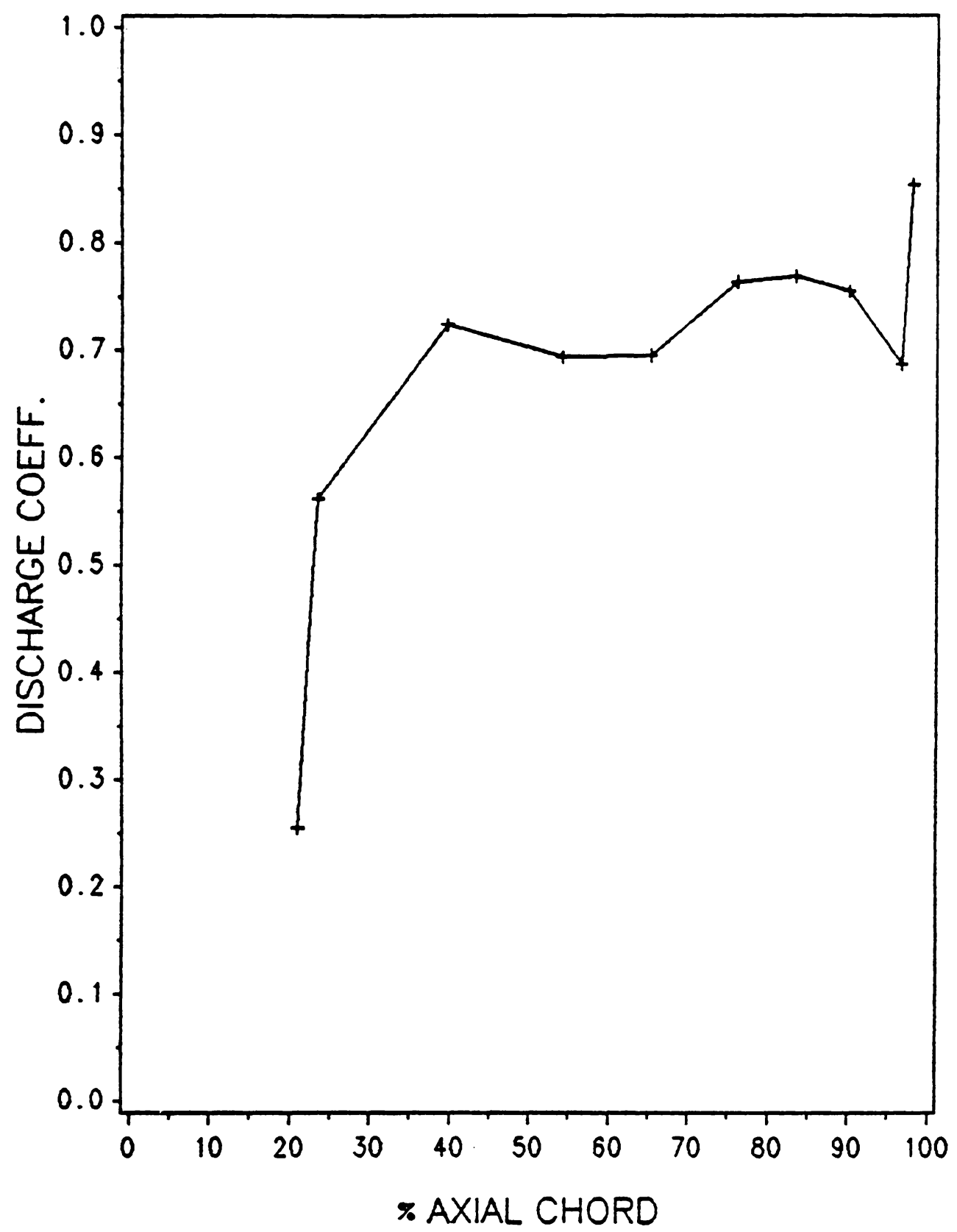

Figure 33. Discharge Coefficients at Tip Gap Exit Plane 


$$
\begin{aligned}
& \delta_{\mathrm{t}}=\text { tip gap height } \\
& \mathrm{dz}=\text { incremental span-wise distance }
\end{aligned}
$$

The values are plotted versus percent axial chord in Figure 34 .

These values represent the losses produced by the leakage flow up to the tip gap exit plane. The highest losses are seen to be at the latter half of the blade.

\subsubsection{OVERALL TIP LEAKAGE COEFFICIENTS}

Various flow properties were integrated over the entire tip gap exit length to develop tip leakage coefficients. The coefficients and their definitions are given below.

Flow Coefficient:

$$
\mathrm{FC}=\frac{\int_{0}^{S_{\mathrm{b}}} \int_{0}^{\delta_{\mathrm{t}}} \rho \mathrm{u}_{\mathrm{n}} \mathrm{dzds}}{\rho \mathrm{Q}}
$$

Total Kinetic Energy Coefficient: 


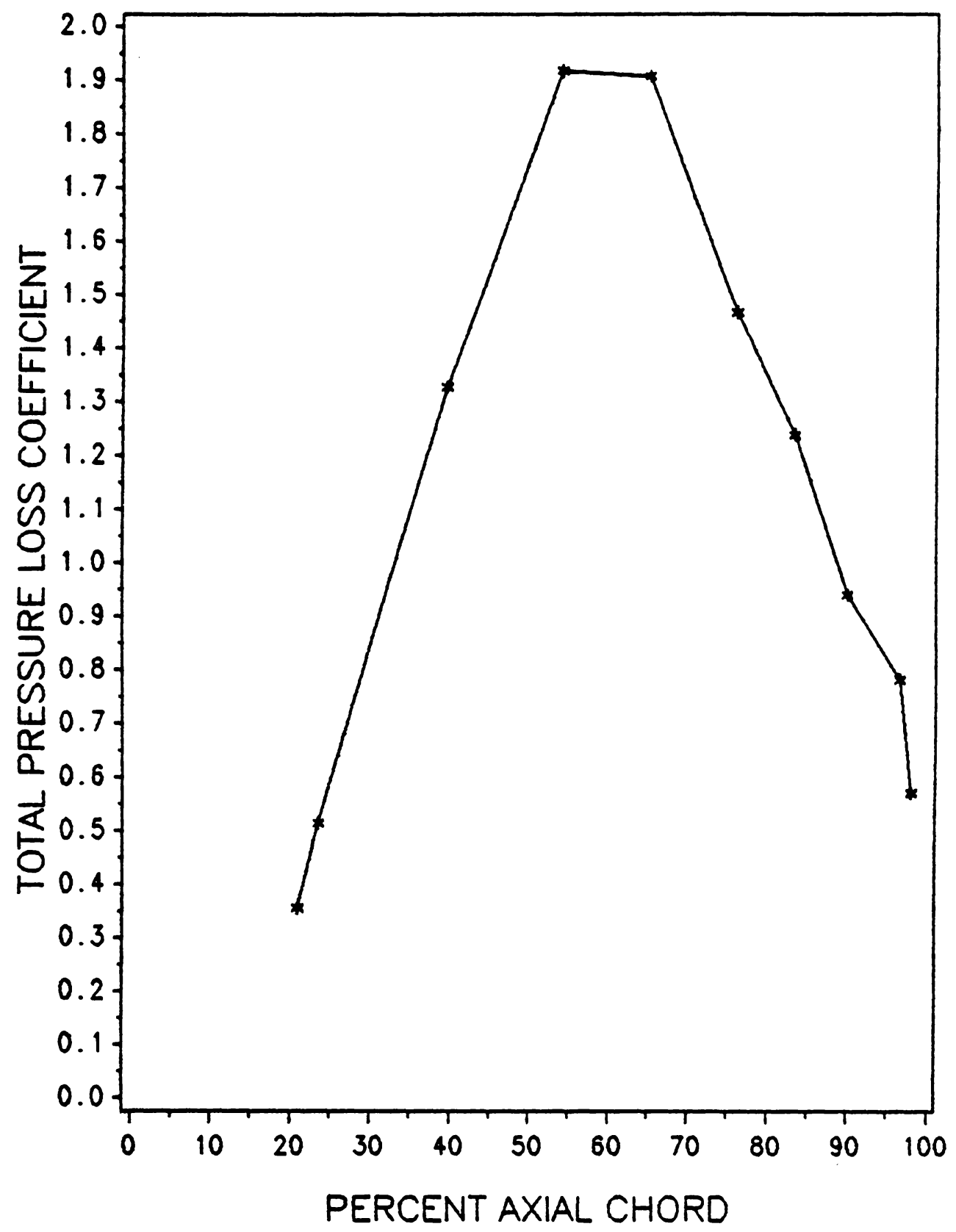

Figure 34. Mass-averaged Total Pressure Loss Coefficients at the Tip Gap Exit Plane 


$$
T K E C=\frac{\int_{0}^{S_{b}} \int_{0}^{\delta_{t}} \rho u_{n}\left[\frac{u_{n f d}^{2}+u_{s f d}^{2}}{2}\right] d z d s}{\rho Q \frac{U_{0}^{2}}{2}}
$$

Normal Kinetic Energy Coefficient:

$$
N K E C=\frac{\int_{0}^{S_{b}} \int_{0}^{\delta_{t}} \rho u_{n}\left[\frac{u_{n f d}^{2}}{2}\right] d z d s}{\rho Q \frac{U_{0}^{2}}{2}}
$$

Streamwise Kinetic Energy Coefficient:

$$
S K E C=\frac{\int_{0}^{s_{b}} \int_{0}^{\delta_{t}} \rho u_{n}\left[\frac{u_{s f d}^{2}}{2}\right] d z d s}{\rho Q \frac{U_{0}^{2}}{2}}
$$

Total Pressure Loss Coefficient: 


$$
T P L C=\frac{\int_{0}^{S_{b}} \int_{0}^{\delta_{t}} \rho u_{n}\left(P_{t 0}-P_{t}\right) d z d s}{\rho Q \frac{U_{0}^{2}}{2}}
$$

where: $Q$ = passage flow rate

$$
\begin{aligned}
& S_{b}=\text { tip gap exit length } \\
& n f d=\text { normal to primary flow direction } \\
& \mathrm{sfd}=\text { tangent to primary flow direction } \\
& \mathrm{ds}=\text { differential tip gap length }
\end{aligned}
$$

The integrations around the blade profile were done so as to account for all the flow exiting the tip gap. This required an estimate of the location where flow first began to exit the tip gap. From the endwall flow visualization, a distance of $3.2 \mathrm{~mm}$ ( $1 / 8$ in ) in front of location 1 was selected. The exact distance turned out to be insignificant because the amount of flow exiting the tip gap at location 1 was so small. Accounting for the flow at the trailing edge was more difficult because of the blade's geometry. On the trailing edge circle, the tangent to the blade surface is not in the primary flow direction as elsewhere on the blade, the primary flow direction on the trailing edge circle being the direction of the mean camber line at the trailing edge. Consulting the endwall flow visualization, the direction of the flow exiting the tip gap around the trailing edge circle appeared to be nearly uniform. With the additional assumption of a uniform velocity distribution, the required velocities could be calculated at any point on the trailing edge circle using the data measured at location 10. 
The curvature of the trailing edge circle meant that the blade surface normal, and therefore the amount of flow exiting the tip gap, changed rapidly. Thus, integration points were chosen every $10^{\circ}$ beginning on the suction side, $90^{\circ}$ from the camber line, and ending on the pressure side, $50^{\circ}$ from the camber line, where the flow ceased to exit the tip gap.

The integrated values of the tip leakage coefficients are presented below.

$$
\begin{aligned}
& \text { FC }=0.057 \\
& \text { TKEC }=0.128 \\
& \text { NKEC }=0.109 \\
& \text { SKEC }=0.020 \\
& \text { TPLC }=0.071
\end{aligned}
$$

These coefficients reveal important information about the tip leakage flow. $5.7 \%$ of the passage flow is seen to pass through the tip gap. The total massaveraged kinetic energy of this flow is $12.8 \%$ of the inlet kinetic energy on a per passage basis. More importantly, the majority of this kinetic energy is exiting the tip gap in a direction normal to the primary passage flow. According to the work of Moore and Adhye [ 16 ], it therefore is expected to be dissipated as the flow mixes out. The streamwise kinetic energy is small and not expected to dissipate as a loss. It must be noted, however, that a small portion of this streamwise kinetic energy is flowing in a direction opposite that of the primary flow; only $1.2 \%$ of the SKEC or a coefficient of 0.0002 is oppositely directed and should therefore have a negligible effect on the overall flow. A significant total pressure loss is seen to have occurred within the tip gap. The mass averaged total pressure loss per passage is $7.1 \%$ of the inlet dynamic pressure. 


\subsection{LOSS PRODUCTION UP TO THE TIP GAP EXIT}

The losses produced up to the tip gap exit in this cascade result from the combined effects of a variety of factors. The flow mixing which occurs within the tip gap is expected to be one of the most significant. An estimate of the maximum mixing loss can be made by treating the tip leakage as a two dimensional potential flow to a vena-contracta followed by mixing to fill the tip gap, see Figure 35 .

Following the analysis of Moore and Tilton [ 1 ], the upstream total pressure is assumed to be the cascade inlet total pressure, $P_{t 0}$, and complete mixing to the measured exit plane pressure is assumed to have occurred. Using Bernoulli's equation from upstream to the vena-contracta:

$$
P_{t 0}=P_{\min }+\frac{1}{2} \rho U_{\max }^{2}
$$

or

$$
1-C_{p s, \min }=\frac{U_{\max }^{2}}{U_{0}^{2}}
$$

Between the vena-contracta and the tip gap exit plane, mixing occurs.

continuity:

$$
U_{\mathrm{ex}}=\sigma U_{\max }
$$




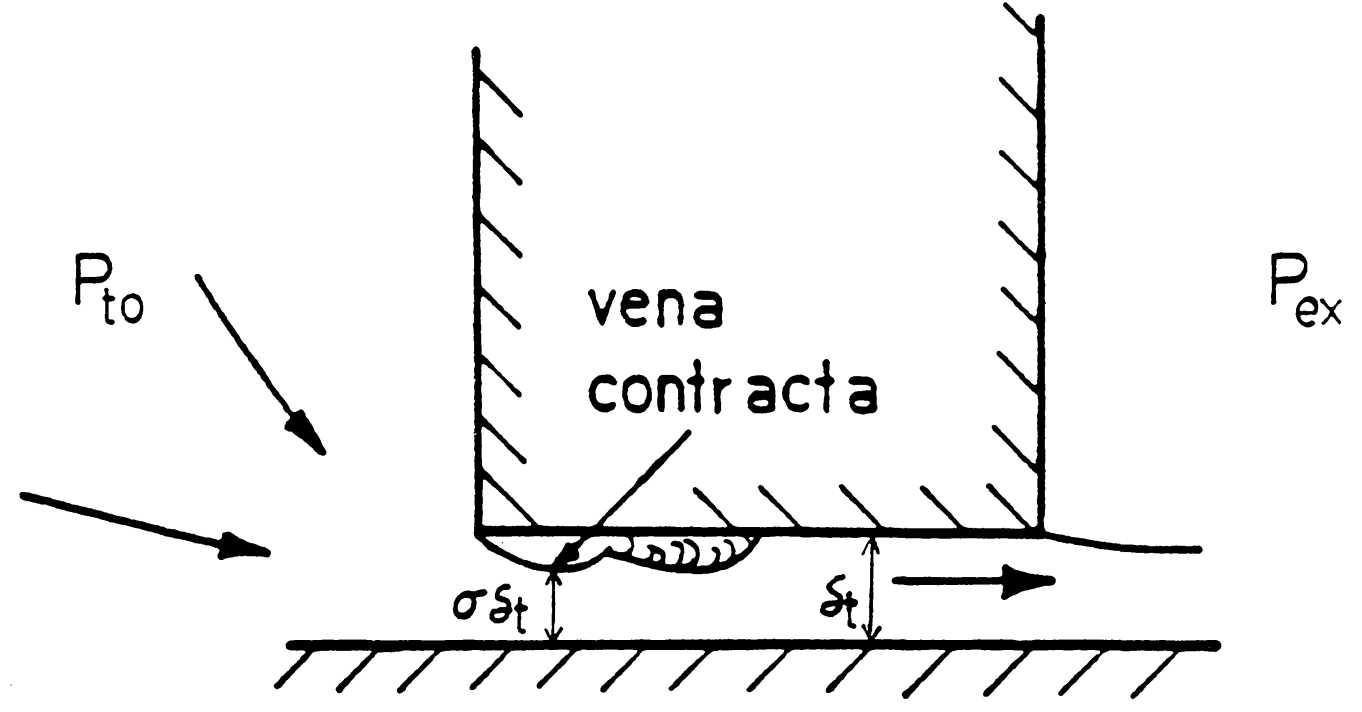

Figure 35. Tip Leakage Flow Model 
momentum:

$$
\mathrm{P}_{\mathrm{ex}}-\mathrm{P}_{\min }=\rho \sigma \mathrm{U}_{\max }^{2}-\rho \mathrm{U}_{\mathrm{ex}}^{2}
$$

or

$$
C_{p s, e x}-C_{p s, \min }=2\left(\sigma-\sigma^{2}\right) \frac{U_{\max }^{2}}{U_{0}^{2}}
$$

Combining equations 6.12 through 6.14 and using a contraction coefficient $\sigma=0.611$ for a shape edged orifice, the total pressure loss due to mixing becomes:

$$
\overline{\bar{C}}_{p t}=0.288\left(1-C_{p s, e x}\right)
$$

It must be remembered that this formula gives the tip gap total pressure loss for complete mixing to the measured exit static pressure. It assumes negligible skin friction and no loss prior to the vena-contracta.

The mass-average total pressure loss coefficients shown in Figure 34 are repeated in Figure 36 along with the values predicted by equation 6.15 .

The predicted values are seen to be high near the blade leading and trailing edges and low elsewhere. The fact that the actual loss over most of the blade is greater than that predicted for mixing suggests that additional loss mechanisms are present. These probably include viscous shear and the possible effects of the laminar separation and turbulent reattachment which were seen to occur within the tip gap (Figures 11 and 12 ). 


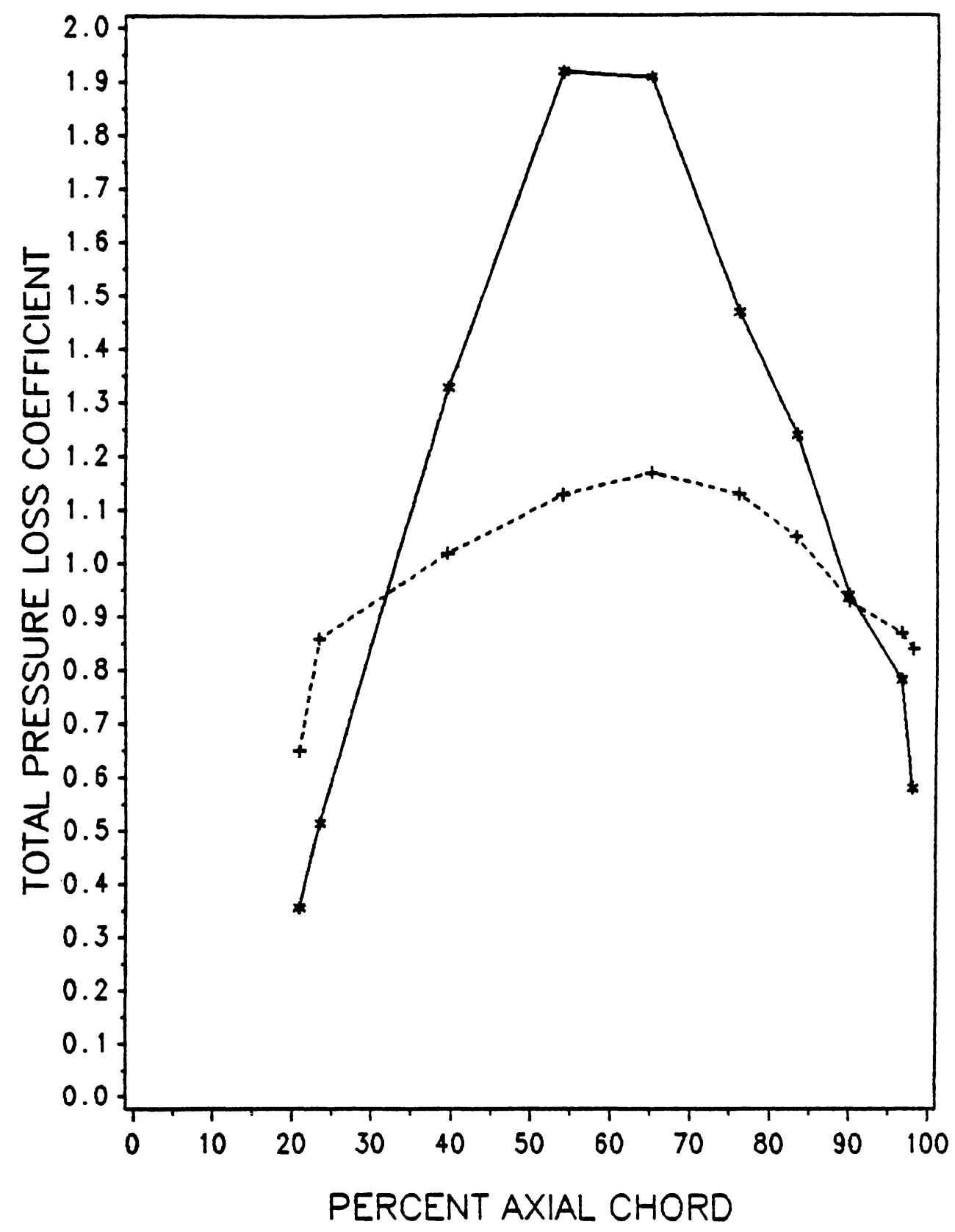

Figure 36. Calculated and Measured Total Pressure Loss Coefficients: - measured; - - calculated, Eq. 6.15 
The extent of the mixing as well as the viscous loss is dependent on the length-to-tip gap height ratio, $\frac{\ell}{\delta_{\mathrm{t}}}$, of the leakage flow path. Using the measured exit plane flow angles and the bottom endwall flow visualization, the length of the flow path within the tip gap for each measurement location was determined. The $\frac{\ell}{\delta_{t}}$ ratio is plotted for each location around the blade profile in Figure 37.

In general, a greater $\frac{\ell}{\delta_{\mathrm{t}}}$ allows more complete mixing and a greater friction loss.

Over most of the blade, there is a distinct correlation between the length of the leakage flow path within the tip gap and the degree of flow mixing at the tip gap exit plane. With reference to Figures 24 and 25, the degree of mixing can be qualitatively judged by the uniformity of the total pressure profiles. At location 2 , where $\frac{\ell}{\delta_{\mathrm{t}}}$ is greatest ( 15.6 ), the mixing appears to be fairly complete. As the trailing edge is approached, $\frac{\ell}{\delta_{t}}$ decreases and the degree of mixing is seen to decrease. This trend continues until near location 8 where $\frac{\ell}{\delta_{\mathrm{t}}}$ and therefore the degree of mixing begin to rise again. As discussed earlier in section 6.2.4, the overall loss level is influenced by the exit plane static pressure. The lower the exit plane static pressure, the greater the driving force of the tip gap flow and the greater the potential for loss production in the tip gap. The $\frac{\ell}{\delta_{\mathrm{t}}}$ ratio simply determines how much of the potential mixing losses are actually incurred within the tip gap exit.

There are several other factors which could affect the overall tip gap losses and their distribution around the blade profile. The suction surface blade length is longer than that of the pressure surface and therefore the flow should diffuse as it passes through the tip gap. This reduction in velocity would increases the residence time within the tip gap and allow more time for mixing to occur. This effect is most prominent near the blade leading edge where the curvature is greatest. Also, the separation and reattachment which occurs on the endwall within the tip gap could 


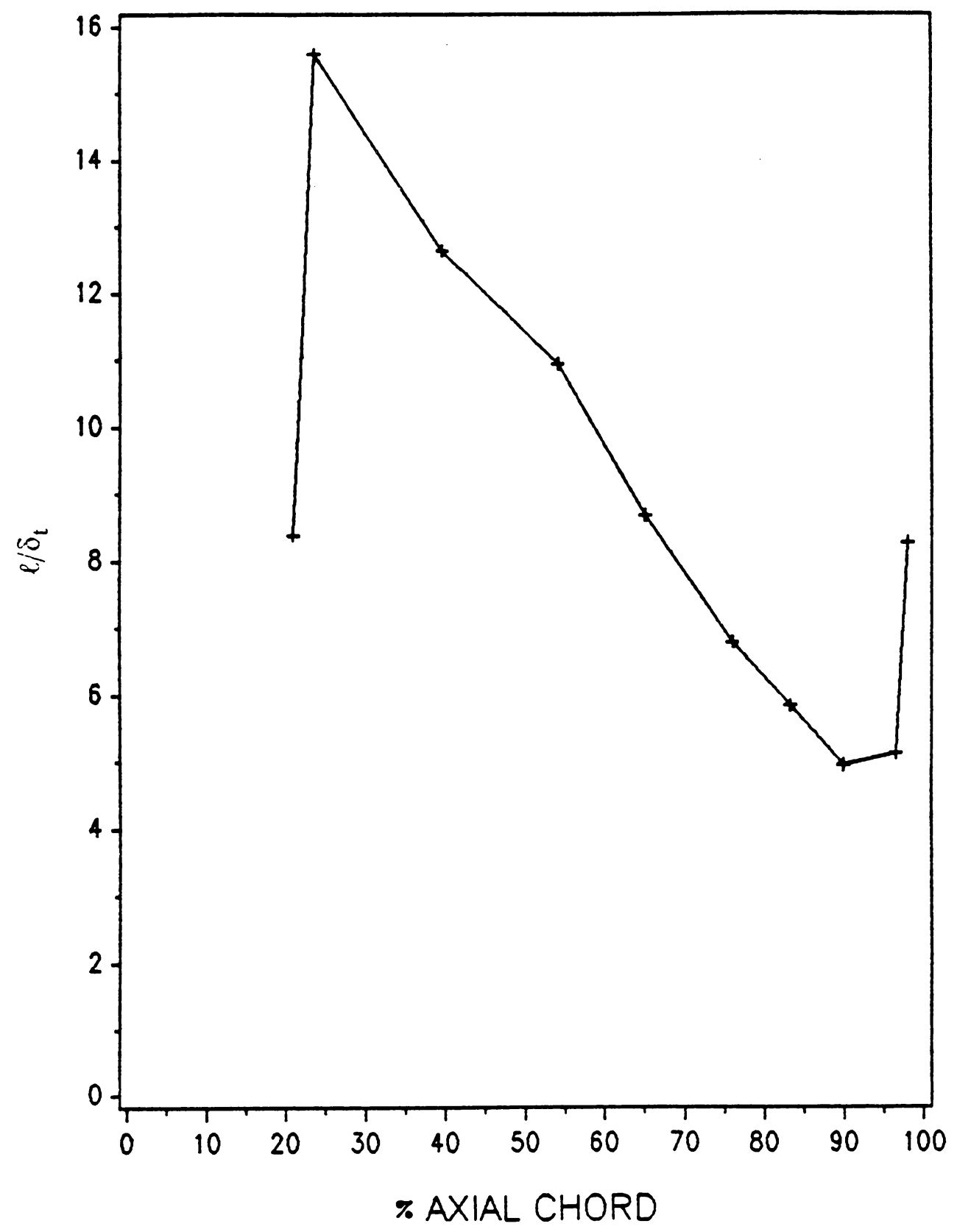

Figure 37. Length to Tip Gap Height Ratios 
cause additional loss. The lack of this phenomenon near the leading edge could partially explain the extremely low level of loss at locations 1 and 2 .

\subsection{DOWNSTREAM MEASUREMENTS}

\subsubsection{SECONDARY FLOW VELOCITIES}

The primary flow direction at the downstream measuring plane $(\mathrm{x} / \mathrm{c}=1.4)$ is defined as the direction of the mean camber line at the blade trailing edge. The secondary velocities, $v$ and $w$, lie in a plane which is perpendicular to the primary flow direction. This cross-sectional plane along with the three velocity components and the yaw and pitch angles are illustrated in Figure 38.

The secondary velocity vectors projected to the cross-sectional plane are presented in Figure 39.

Because the three-hole probes used for the near-wall measurements could not distinguish spanwise velocities, the $w$ velocities from the near-wall five-hole probe measurements were imposed on the overlapping three-hole probe measurements. The $w$ velocity was then assumed to vary linearly to zero at the walls.

Flow in the bottom half of the passage is dominated by the tip leakage vortex. At this downstream distance, it contains flow with secondary velocities reaching 11.2 $\mathrm{m} / \mathrm{s}, 0.55 \mathrm{U}_{0}$. A smaller passage vortex with maximum secondary velocities of about $0.18 U_{0}$ is seen in the top half of the passage. The rotation of a passage vortex is not 


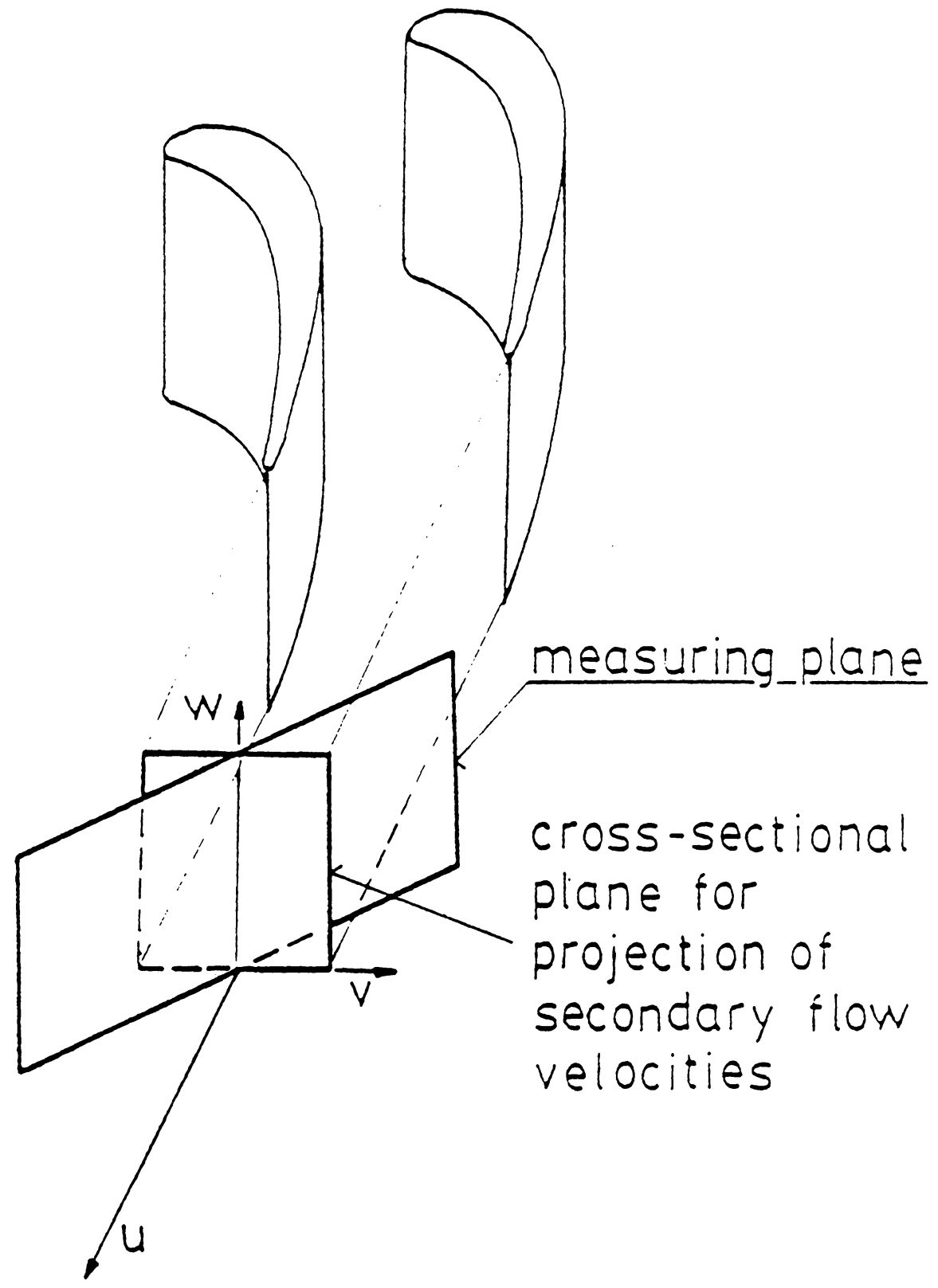

Figure 38. Cross-Sectional Plane and Coordinate System 


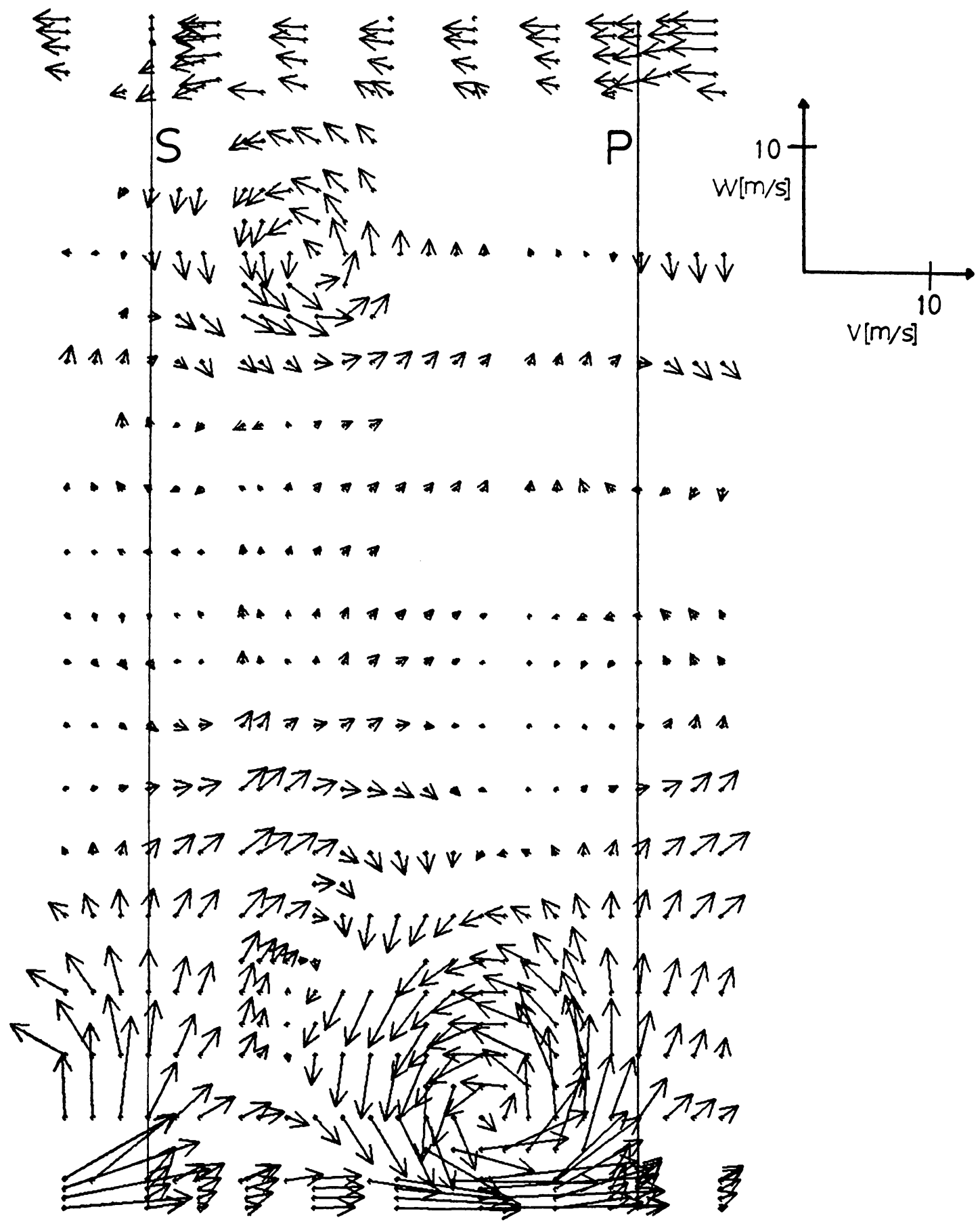

Figure 39. Secondary Velocity Vectors in the Cross-sectional Plane at $x / c=1.4:$ - projections downstream of blades in direction of mean camber lines at trailing edges; $S$ - downstream of blade $3, P$ - downstream of blade 2 
clearly seen in the bottom half of the passage although some variations in the secondary velocities are seen at a corresponding location. These fluctuations could be a passage vortex which has been distorted by interaction with the leakage vortex. Also in evidence is the expected overturning of the top endwall boundary layer.

\subsubsection{TOTAL PRESSURE}

Contours of constant total pressure loss coefficient, $C_{p t}$ ( equation 6.1 ), are shown in Figure 40.

In addition to the blade wakes, three distinct regions of high loss are present. The strongest of these is the tip leakage vortex having a maximum $C_{p t}$ of 2.8 . The passage vortex in the top half of the passage is easily distinguished but considerably weaker having a maximum loss coefficient of 1.1 . The third high loss core has a maximum $C_{p t}$ of 1.3 , slightly higher than that of the top passage vortex.

The similar strength and symmetric location of this third loss core with the top passage vortex suggests that it is the result of a second passage vortex. However, as mentioned previously, the secondary velocity vector plot does not show clear evidence of this. Superimposing the secondary velocity vectors on the total pressure contours in the measurement plane gives a better perspective on this situation, see Figure 41.

The velocity vectors show secondary flow from the leakage vortex in the adjacent passage breaking away and moving into this high loss region. The endwall boundary layer is also seen to be lifted up and carried by the escaping fluid. Alternatively, it could be suggested that the the rotation of a passage vortex is present in the bottom half of the passage. Near the center of the third high loss core, the secondary ve- 


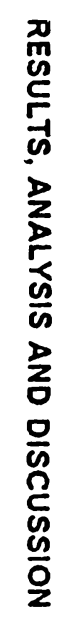

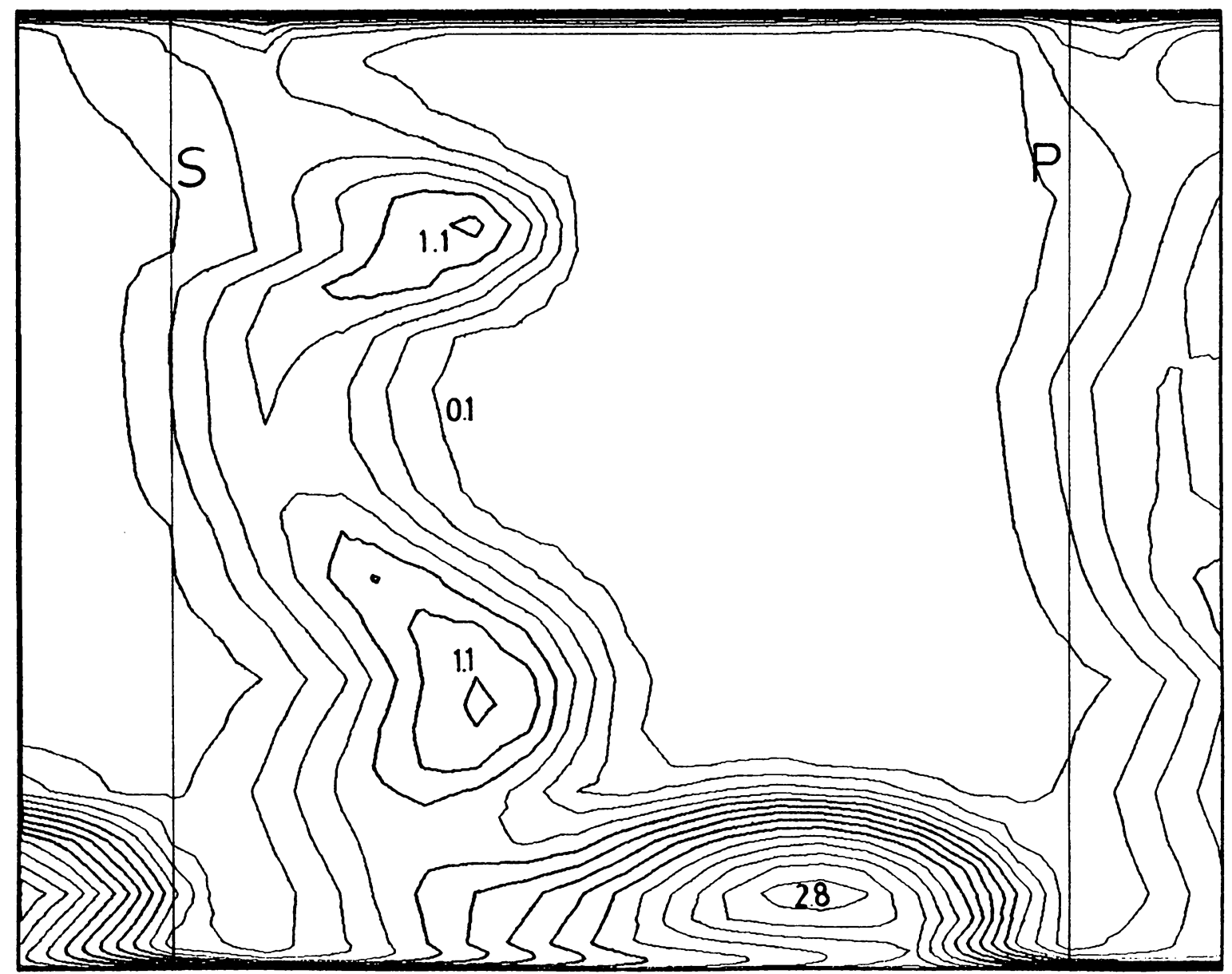

Figure 40. Contours of Constant Total Pressure Loss Coefficient: Measurement Plane at $\mathrm{x} / \mathrm{c}=$ 


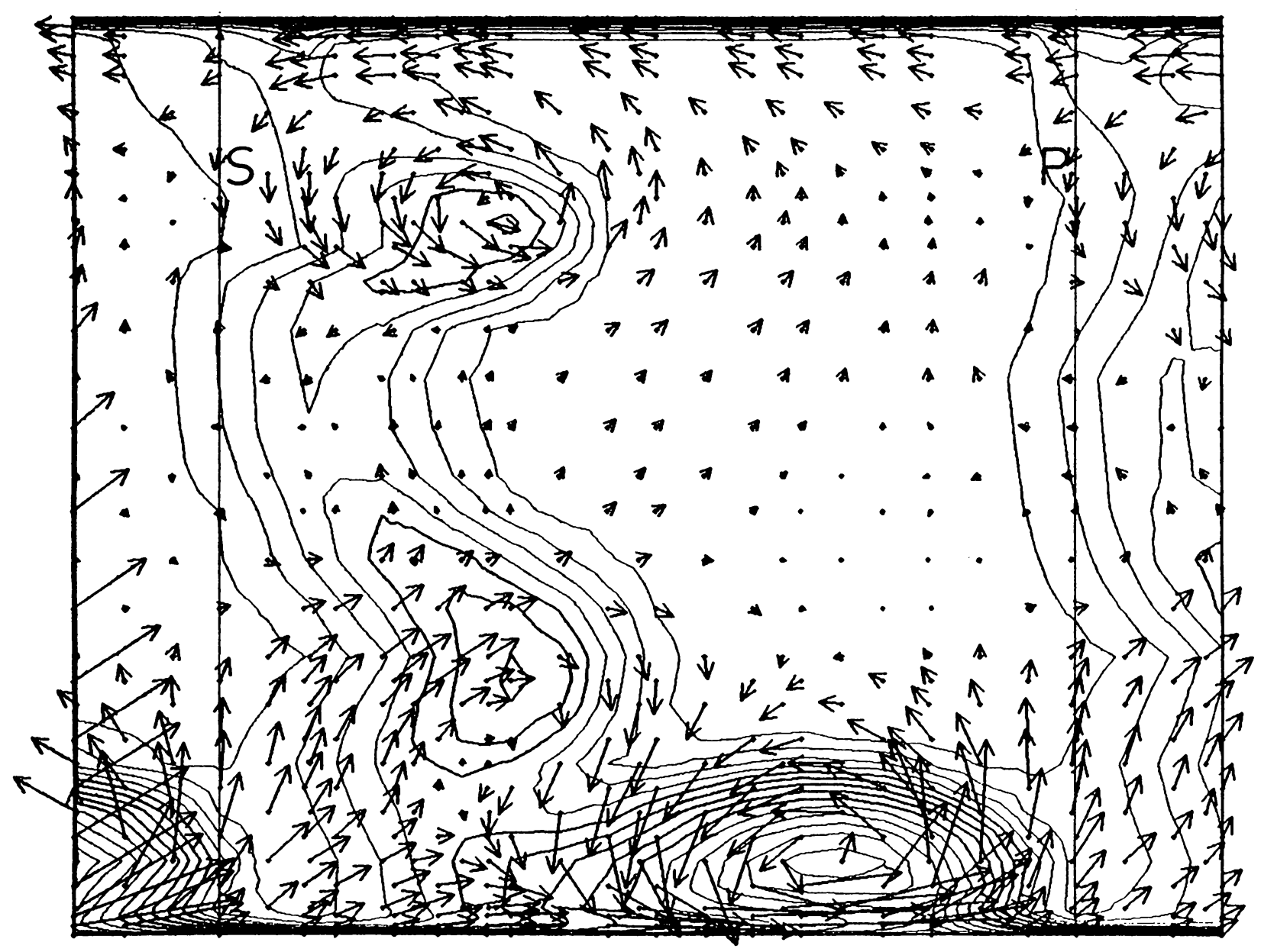

Figure 41. Secondary Velocities and Total Pressure Loss Coefficients: Measurement Plane at $x / c=1.4$ 
locity vectors do show a clockwise rotation, but it breaks down when it encounters the leakage vortex. This could be construed as due to a passage vortex which, upon interacting with the leakage vortex, has its secondary velocities redirected.

Another interesting observation which can be made from Figure 41 is the close agreement of the center of rotation with the area of highest loss in both the passage and leakage vortices.

The top endwall boundary layer is seen to be quite thin, extending only $6 \rightarrow 7$ $\mathrm{mm}$ into the passage. The boundary layer on the bottom wall appears even thinner, although its full extent is difficult to distinguish because of the presence of the leakage vortex and blade wakes. Estimates of its thickness range from less than 2 $\mathrm{mm}$ under the leakage vortex to $5 \mathrm{~mm}$ near the blade wakes.

A significant area of low loss $\left(C_{p t}<0.1\right)$ is seen to exist within the passage. This region which covers about half the flow area represents nearly two-dimensional flow. Near mid-span, the blade wake seems undisturbed by the three loss cores and could be a measure of the blade profile loss.

\subsubsection{STATIC PRESSURE}

The static pressures near the endwalls could not be measured with the three-hole probes. The near-wall static pressures were therefore linearly interpolated from the endwall values to the pressures of the near-wall five-hole probe traverses.

The contours of static pressure coefficient, $C_{p s}$ ( equation 6.2 ), are shown in Figure 42. 


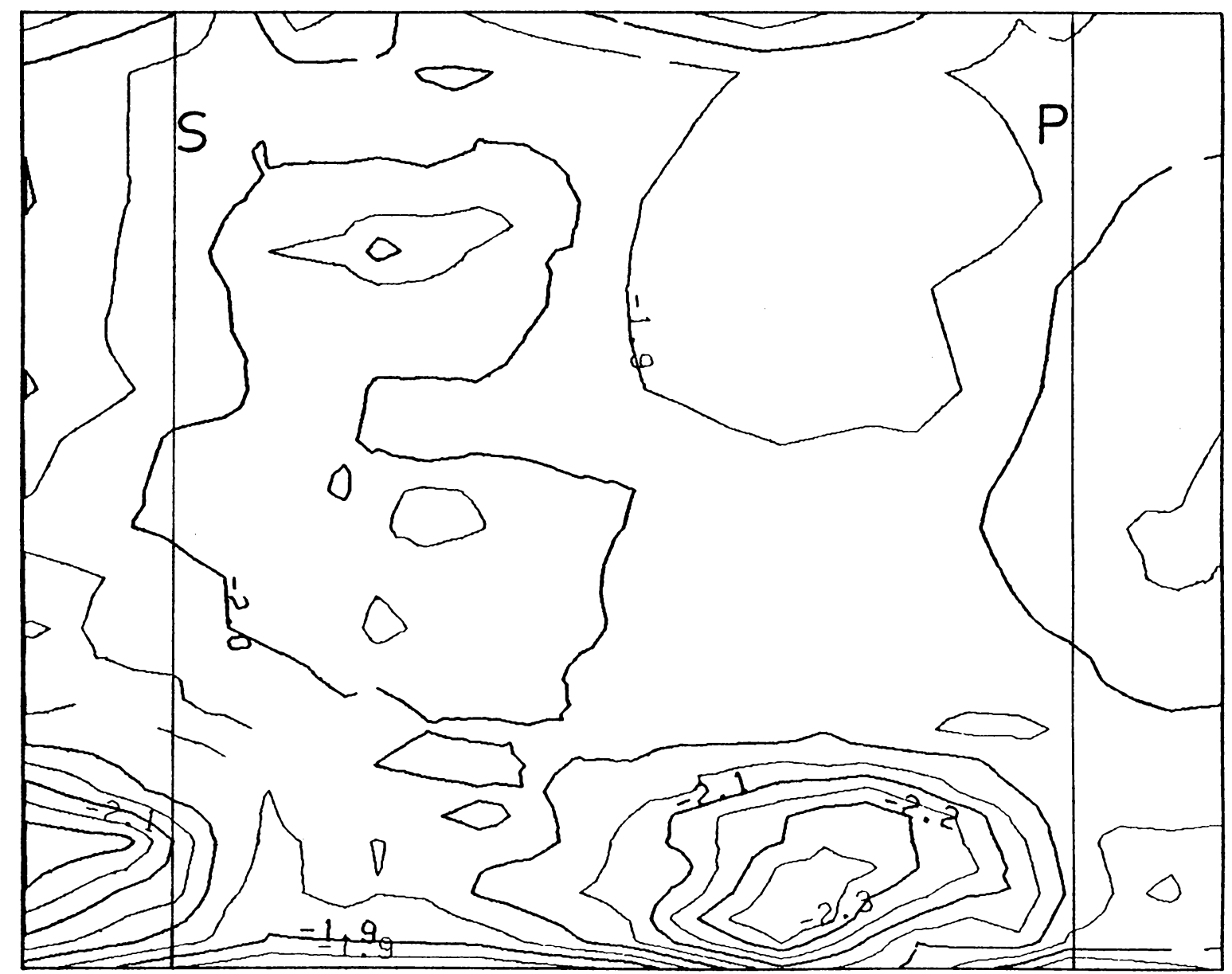


The static pressure at this plane is seen to be fairly uniform ( $\left.C_{p s} \sim-2.0\right)$ The only major zone of low pressure is near the center of the leakage vortex where $C_{p s}$ drops to -2.25 . An area of depressed static pressure is also present near the center of the top passage vortex. Its small area of influence and minimum $C_{p s}$ of only -2.1 is indicative of its lesser strength in relation to the leakage vortex. A similar but less distinct area corresponds to the third high loss core.

\subsubsection{AVERAGED FLOW PROPERTIES}

Flow properties such as velocity components, flow angle, total pressure loss coefficient and kinetic energy coefficients were averaged over the downstream plane on a mass and area basis. These quantities and their definitions are given below.

Velocity components, see Figure 43:

$$
\bar{V}_{n}=\frac{\int_{0}^{\Delta Y} \int_{0}^{\Delta Z} V_{n} d z d y}{\Delta Y \Delta Z}
$$




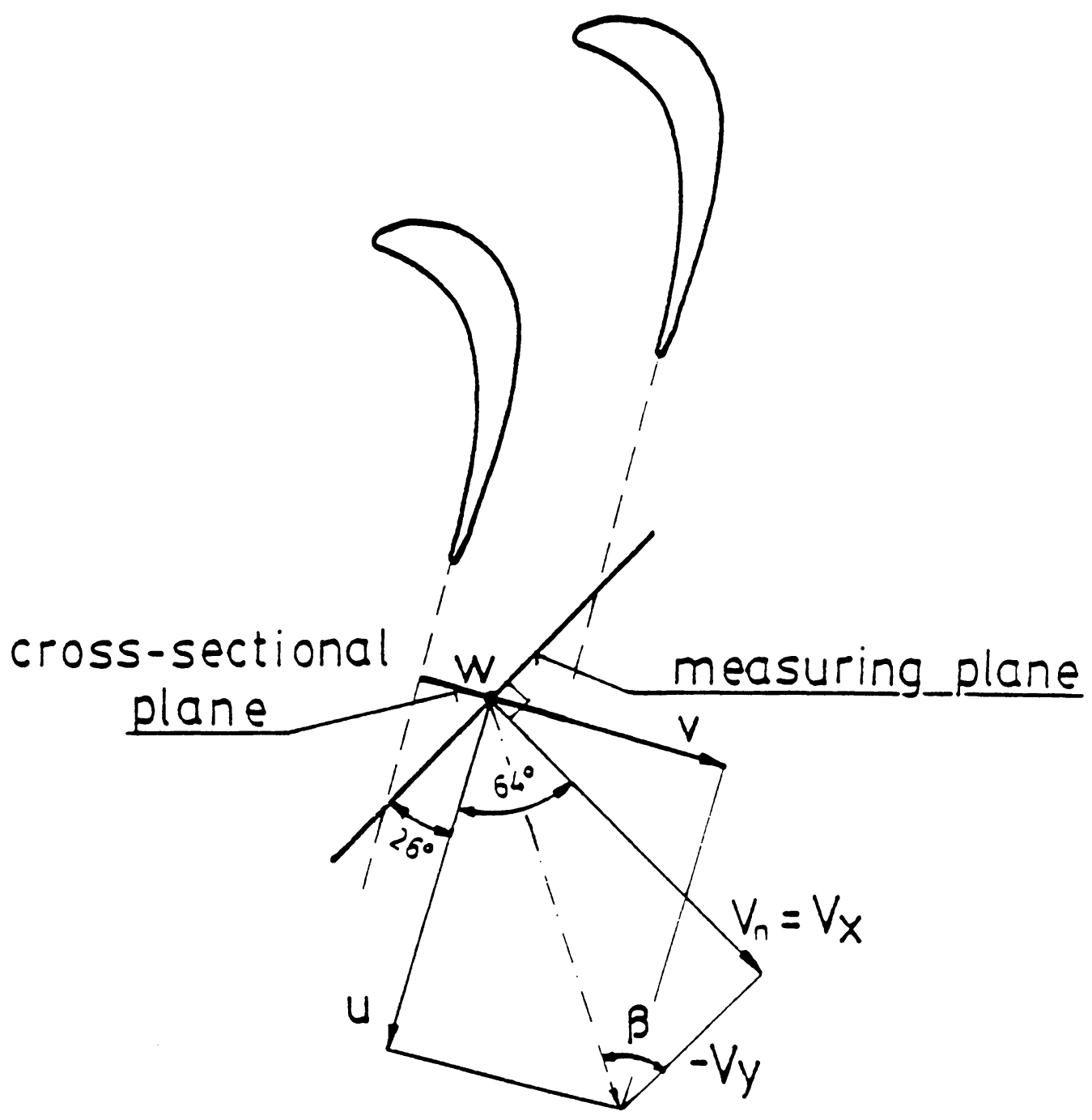

Figure 43. Flow Coordinate System at Downstream Measuring Plane 


$$
\overline{\bar{V}}_{\mathrm{x}}=\frac{\int_{0}^{\Delta Y} \int_{0}^{\Delta z} \rho V_{n} V_{x} d z d y}{\int_{0}^{\Delta Y} \int_{0}^{\Delta Z} \rho V_{n} d z d y}
$$

$$
\overline{\bar{V}}_{y}=\frac{\int_{0}^{\Delta Y} \int_{0}^{\Delta z} \rho V_{n} V_{y} d z d y}{\int_{0}^{\Delta Y} \int_{0}^{\Delta z} \rho V_{n} d z d y}
$$

Averaged flow angles:

$$
\begin{aligned}
& \bar{\beta}=\tan ^{-1}\left[\frac{\bar{V}_{x}}{-\overline{\bar{V}}_{y}}\right] \\
& \overline{\bar{\beta}}=\tan ^{-1}\left[\frac{\overline{\bar{V}}_{x}}{-\overline{\bar{V}}_{y}}\right]
\end{aligned}
$$

Total pressure loss coefficient: 


$$
\overline{\bar{C}}_{p t}=\frac{\int_{0}^{\Delta Y} \int_{0}^{\Delta z} \rho V_{n} C_{p t} d z d y}{\int_{0}^{\Delta Y} \int_{0}^{\Delta z} \rho V_{n} d z d y}
$$

Kinetic energy coefficients:

$$
\frac{\overline{\mathrm{u}}^{2}}{\mathrm{U}_{0}^{2}}=\frac{\int_{0}^{\Delta Y} \int_{0}^{\Delta z} \rho V_{n}\left[\frac{u^{2}}{2}\right] d z d y}{\frac{U_{0}^{2}}{2} \int_{0}^{\Delta Y} \int_{0}^{\Delta z} \rho V_{n} d z d y}
$$

$$
\frac{\overline{\bar{v}}^{2}}{\mathrm{U}_{0}^{2}}=\frac{\int_{0}^{\Delta Y} \int_{0}^{\Delta z} \rho V_{n}\left[\frac{v^{2}}{2}\right] d z d y}{\frac{U_{0}^{2}}{2} \int_{0}^{\Delta Y} \int_{0}^{\Delta z} \rho V_{n} d z d y}
$$




$$
\frac{\overline{\bar{W}}^{2}}{U_{0}^{2}}=\frac{\int_{0}^{\Delta Y} \int_{0}^{\Delta z} \rho V_{n}\left[\frac{w^{2}}{2}\right] d z d y}{\frac{U_{0}^{2}}{2} \int_{0}^{\Delta Y} \int_{0}^{\Delta z} \rho V_{n} d z d y}
$$

where $\quad V_{n}=$ velocity component normal to measuring plane

$V_{x}=$ axial component of velocity (Figure 43)

$V_{y}=$ velocity component in blade to blade direction

$\mathrm{u}=$ velocity component in primary flow direction

$w=$ velocity component in spanwise direction

$v$ = velocity component orthogonal to $u$ and $w$

$\Delta Z=$ passage height

$\Delta Y=$ blade pitch

$d z=$ differential spanwise direction

$d y=$ differential pitchwise direction

To perform the numerical integrations, an interpolation program was used to create a uniform grid of data points. The program allowed either horizontal or vertical interpolation at any point in the flow field. Because most of the interpolations were done in the top half of the passage where gradients of the measured properties were weaker in the spanwise direction, the interpolations were performed vertically wherever possible. The large number of actual data points in the areas of large flow variation should insure the accuracy of the interpolated points. 
Using the area averaged normal velocity, $\bar{V}_{n}$, at the downstream measurement plane, a mass flow coefficient, $\eta$, was defined.

$$
\eta=\frac{\overline{\mathrm{V}}_{\mathrm{n}}}{\overline{\mathrm{V}}_{\mathrm{n}, \text { cascade inlet }}}
$$

The principle of mass conservation demands that $\eta$ be $100 \%$. However, it is very sensitive to variations in measured yaw angle. A systematic yaw correction of $1.85^{\circ}$ was needed to lower $\eta$ from $106 \%$ to $100 \%$. This correction was applied to each data point and the integrations were repeated. The resulting changes in the integrated values appeared only in the third significant figure but were retained to satisfy continuity over the flow field.

The various properties averaged over the flow field are presented in Table 3. 
Table 3. Averaged Flow Properties

\begin{tabular}{|c|c|}
\hline $\bar{V}_{n}(\mathrm{~m} / \mathrm{s})$ & 14.4 \\
\hline$\overline{\bar{V}}_{x}(\mathrm{~m} / \mathrm{s})$ & 14.6 \\
\hline$\overline{\bar{V}}_{y}(\mathrm{~m} / \mathrm{s})$ & -29.3 \\
\hline $\bar{\beta}\left({ }^{\circ}\right)$ & 26.1 \\
\hline$\overline{\bar{\beta}}\left({ }^{0}\right)$ & 26.6 \\
\hline$\overline{\bar{C}}_{p t}$ & 0.375 \\
\hline$\frac{\overline{\bar{u}}^{2}}{U_{0}^{2}}$ & 2.597 \\
\hline$\frac{\overline{\bar{v}}^{2}}{U_{0}^{2}}$ & 0.010 \\
\hline$\frac{\overline{\overline{\mathrm{W}}^{2}}}{U_{0}^{2}}$ & 0.006 \\
\hline$\frac{\overline{\overline{v^{2}+w^{2}}}}{U_{0}^{2}}$ & 0.016 \\
\hline$\overline{\bar{C}}_{p t}+\frac{\overline{\overline{v^{2}+w^{2}}}}{U_{0}^{2}}$ & 0.391 \\
\hline
\end{tabular}

The area averaged normal velocity $\bar{V}_{n}$ is $14.4 \mathrm{~m} / \mathrm{s}$ as required for mass conservation. The mass averaged flow velocities $\overline{\bar{V}}_{x}$ and $\overline{\bar{V}}_{y}$ are $14.6 \mathrm{~m} / \mathrm{s}$ and $-29.3 \mathrm{~m} / \mathrm{s}$ respectively. By equation 6.19 , the averaged flow angle calculated from these velocities is $\bar{\beta}=$ 26.1 $1^{\circ}$. Because $\bar{V}_{n}$ must remain constant to satisfy continuity, and, if endwall shear is 
negligible, $\overline{\bar{V}}_{y}$ is constant, this definition produces a flow angle which is constant with downstream distance. Alternatively, equation 6.20 , based on the mass-averaged axial velocity allows $\overline{\bar{\beta}}$ to vary with downstream distance as the flow mixes out. At $x / c=$ 1.4 this definition gives $\overline{\bar{\beta}}=26.6^{\circ}$ which indicates a very slight underturning of the flow. It is worth noting that when the flow is completely mixed out, $\overline{\bar{V}}_{\mathrm{x}}=\overline{\mathrm{V}}_{\mathrm{x}}=\overline{\mathrm{V}}_{\mathrm{n}}$ and $\overline{\bar{\beta}}=\bar{\beta}=26.1^{\circ}$ which is essentially the blade exit angle.

The mass averaged total pressure loss coefficient $\overline{\bar{C}}_{p t}$ is 0.375 . An indication of the importance of tip leakage flow in loss production can be gained by comparison with the earlier work of Moore and Adhye [ 16 ]. On a geometrically similar cascade a mass averaged total pressure loss coefficient of 0.347 was recorded for the same downstream measuring plane. In their case, however, the inlet boundary layers were significantly thicker, measuring approximately $39.0 \mathrm{~mm}$ compared to the present 2.2 $\mathrm{mm}$. Their large inlet boundary layers contributed significant loss $\left(\overline{\bar{C}}_{\mathrm{pt}} \sim 0.07\right)$ at the cascade inlet and produced extreme secondary flows within the blade passage. Even with the large high loss passage vortices, the mass-averaged loss at $x / c=1.4$ was less than that of the present cascade with a leakage vortex and two (or one) small passage vortices.

Better insight to the loss distribution within the passage can be gained from pitch-wise and span-wise mass-averaged values of total pressure loss coefficient. Following the work of Moore [ 21 ], two methods of averaging are defined.

Pitch-wise averages: 


$$
\bar{C}_{p t a}=\frac{\int_{0}^{\Delta Y} \rho V_{n} C_{p t} d y}{\frac{1}{\Delta Z} \int_{0}^{\Delta Z} \int_{0}^{\Delta Y} \rho V_{n} d y d z}
$$

$$
\bar{C}_{p t b}=\frac{\int_{0}^{\Delta Y} \rho V_{n} C_{p t} d y}{\int_{0}^{\Delta Y} \rho V_{n} d y}
$$

span-wise averages:

$$
\bar{C}_{p t c}=\frac{\int_{0}^{\Delta Z} \rho V_{n} C_{p t} d z}{\frac{1}{\Delta Y} \int_{0}^{\Delta Z} \int_{0}^{\Delta Y} \rho V_{n} d y d z}
$$

$$
\bar{C}_{p t d}=\frac{\int_{0}^{\Delta z} \rho V_{n} C_{p t} d z}{\int_{0}^{\Delta z} \rho V_{n} d z}
$$


The distributions of $\bar{C}_{p t a}$ and $\bar{C}_{p t b}$ along the passage height are shown in Figures 44 and 45 respectively.

$\overline{\mathrm{C}}_{\mathrm{pta}}$ is a measure of the total pressure loss coefficients at a particular span-wise location averaged over the total passage mass flow. $\overline{\mathrm{C}}_{\mathrm{ptb}}$ is similar except that it is averaged over the local mass flow at the particular spanwise-location. Note that $\bar{C}_{\text {pra }}$ is defined such that the area under its distribution curve is $\overline{\bar{C}}_{p t}$.

The only significant difference in the two figures is that the level of loss accumulated in the tip leakage vortex is greater for $\bar{C}_{p t b}$. This indicates that the relatively small percentage of the total mass flow contained in the leakage vortex accounts for a large percentage of the overall losses. The high loss of the leakage flow is seen extending to about $20 \%$ of the blade span in both figures. The smaller loss accumulation near $75 \%$ span is due to the passage vortex. The thin high loss area of the top endwall boundary layer is also evident. In addition, an area of uniform loss is seen near mid span. This relatively constant pitch-wise averaged total pressure loss coefficient of 1.6 from $50 \%$ to $60 \%$ of the span is believed to be a measure of the profile loss.

The pitch-wise distribution of span-wise averaged total pressure loss coefficients are shown in Figures 46 and 47.

These figures are virtually identical. The major feature is the large region of high loss centered at $25 \%$ of the pitch measured from the suction side of the passage. This peak represents the combined losses of the blade wake, the top passage vortex and the third loss core, see Figure 40. A second but much smaller peak centered around $68 \%$ of the pitch is the tip leakage vortex. The effect of the leakage vortex seems small when averaged in a span-wise sense because all the other fluid at its pitchwise location is of virtually no loss. 


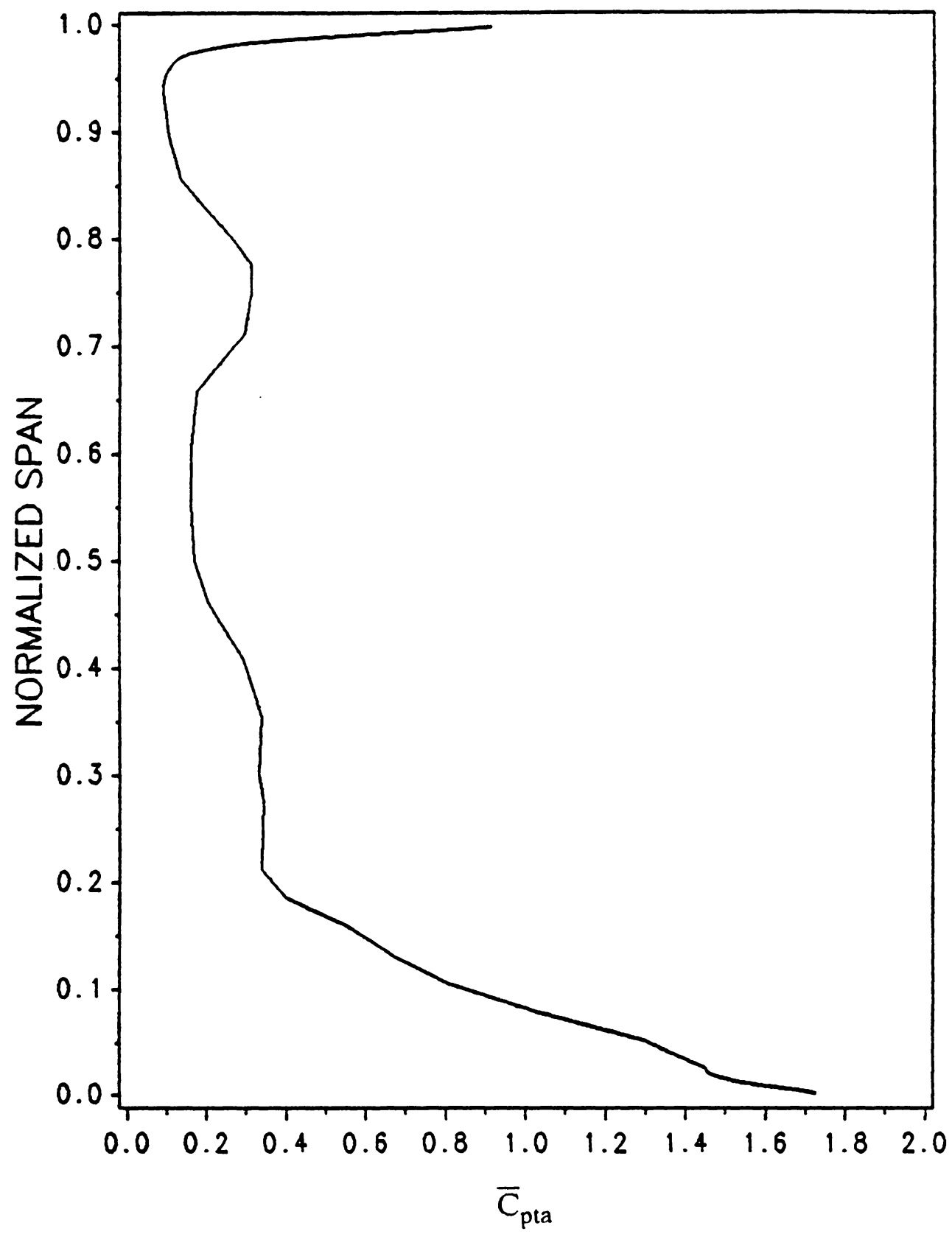

Figure 44. Pitch-wise Averaged Total Pressure Loss Coefficient, Cpta 


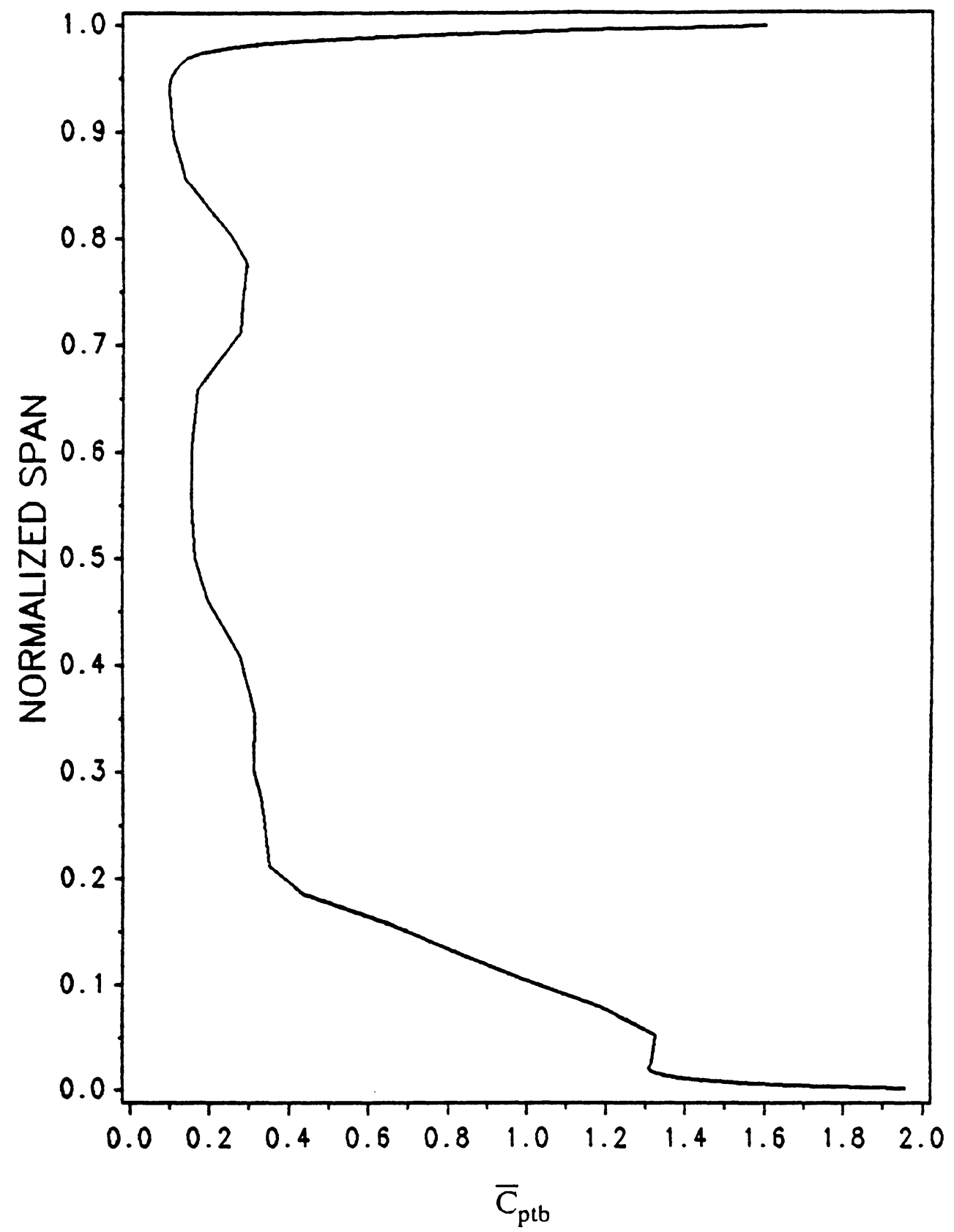

Figure 45. Pitch-wise Averaged Total Pressure Loss Coefficient, Cptb 


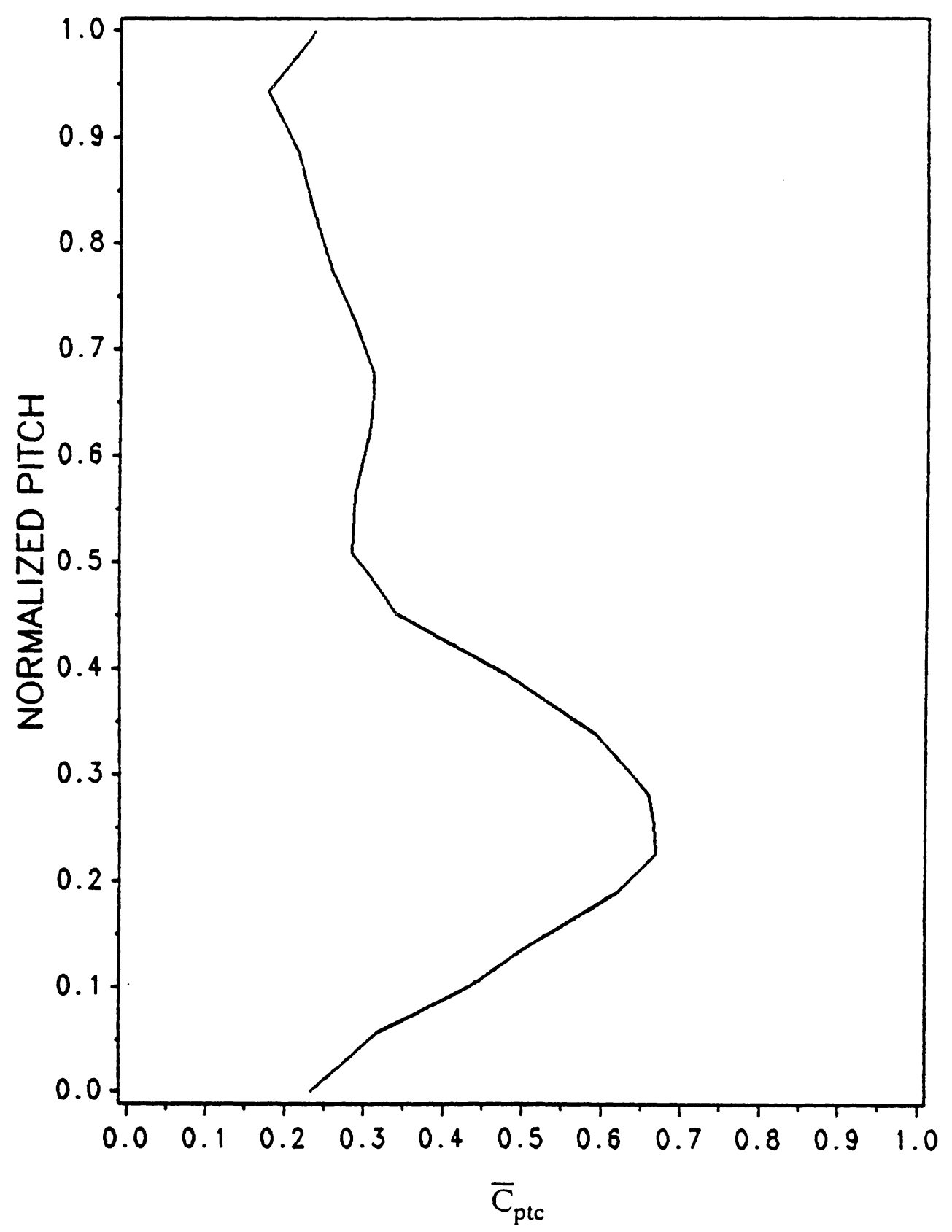

Figure 46. Span-wise Averaged Total Pressure Loss Coefficient, Cptc 


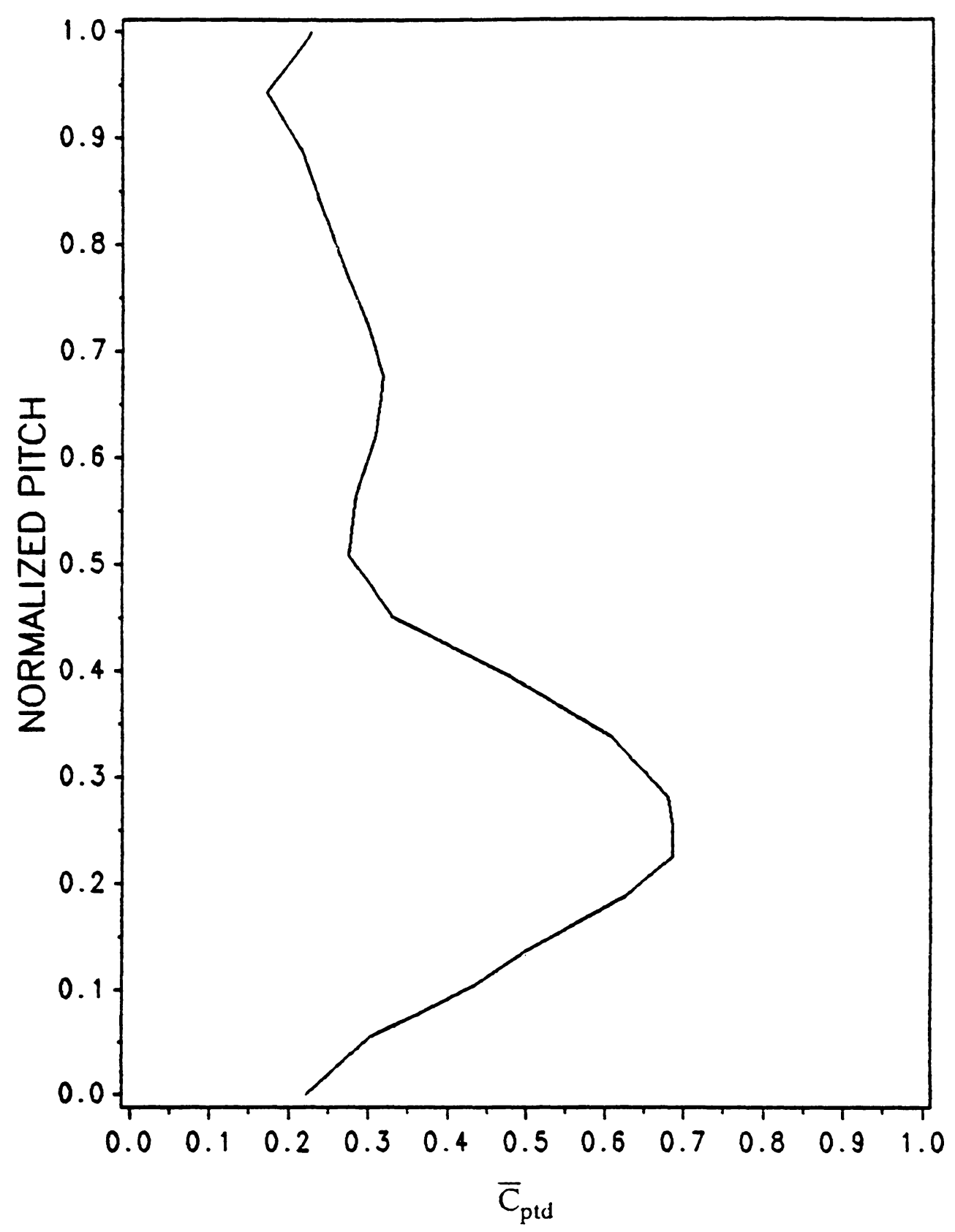

Figure 47. Span-wise Averaged Total Pressure Loss Coefficient, Cptd 
The distribution of pitch-wise-mass-averaged flow angle was also found to be of interest. Figure 48 shows the span-wise variation of pitch-wise-mass averaged flow angle defined as

$$
\overline{\bar{\theta}}=90-\tan ^{-1}\left[\frac{\int_{0}^{\Delta Y} \rho V_{n} V_{x} d y}{\int_{0}^{\Delta Y} \rho V_{n} V_{y} d y}\right]
$$

for a particular span-wise location. Note that $\overline{\bar{\theta}}=90^{\circ}-\overline{\bar{\beta}}$, by definition.

$\overline{\bar{\theta}}$ of $64^{\circ}$ corresponds to the direction of the blade trailing edge mean camber lines.

Near the bottom endwall, the tip leakage vortex produces significant underturning of the flow. The underturning due to the passage vortex as well as the overturning of the top endwall boundary layer are also clearly evident. The flow is seen to be essentially at the exit blade angle between $50 \%$ and $60 \%$ of the span giving further evidence of a profile loss in this area.

\subsubsection{MIXING ANALYSIS}

As an estimate of the maximum possible loss for this cascade, the measured flow at $x / c=1.4$ was numerically mixed with constant area to a uniform static pressure. This was done by solving the continuity and momentum equations over a control volume for one passage flow. The control volume extended from the measurement plane to an arbitary distance downstream where the flow was considered mixed out. A diagram of this control volume is given in Figure 46. 


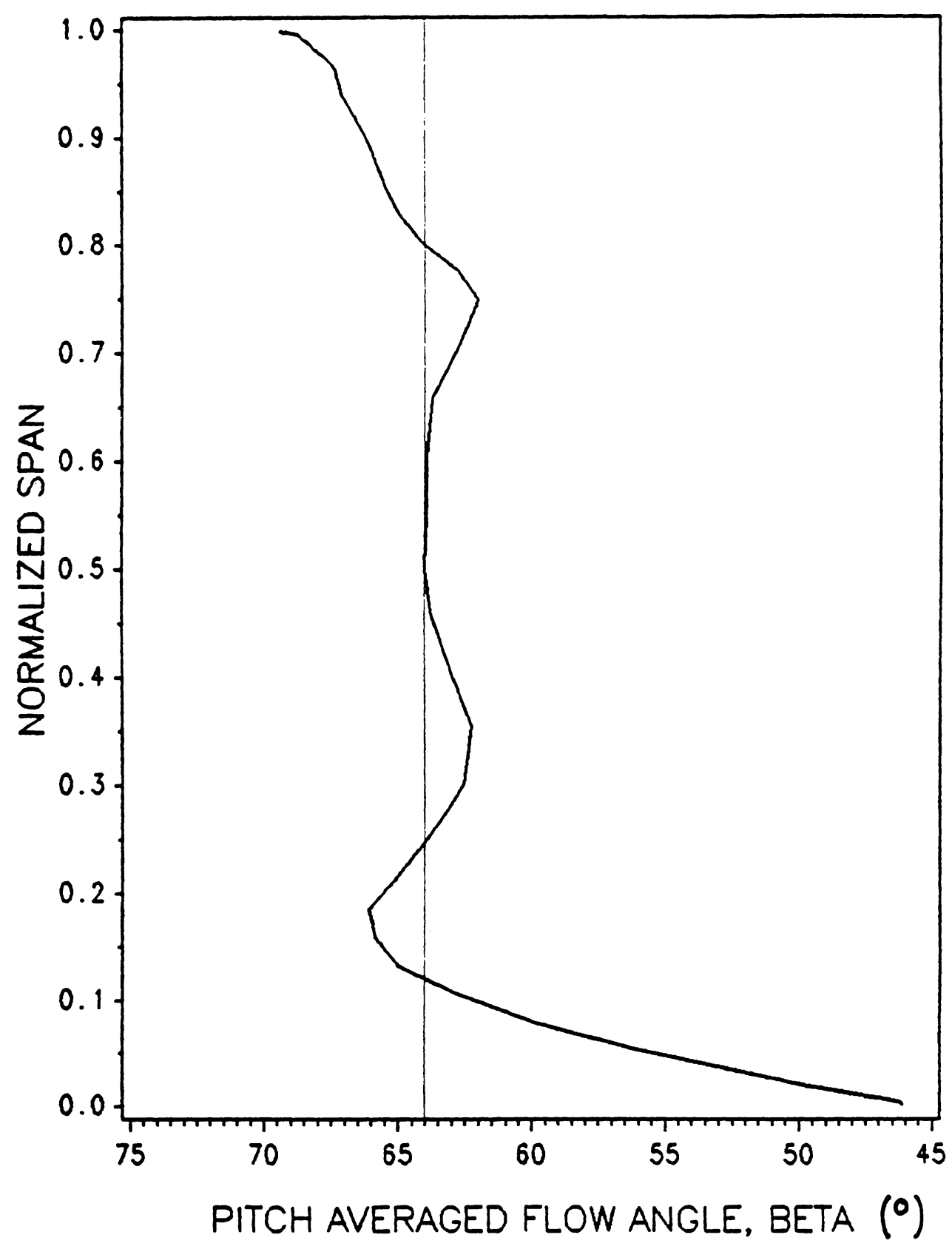

Figure 48. Pitch-wise-mass-averaged Flow Angle 


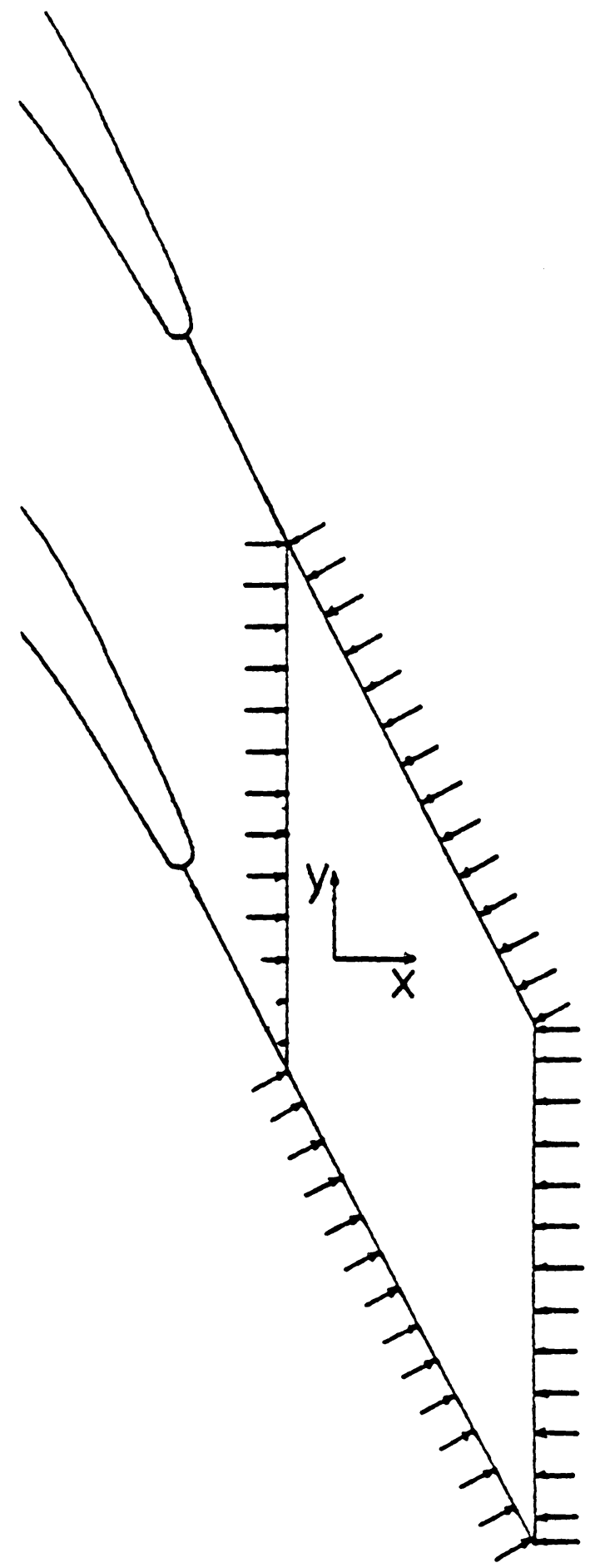

Figure 49. Mixing Control Volume 
Because the cascade is assumed to be infinite, the forces on both sides of the control volume balance each other. In addition, the shear stresses on the endwalls were neglected. Therefore, the only external forces acting on the control volume in this analysis are the static pressures on the upstream and downstream faces. In this case, the upstream face is the measurement plane at $x / c=1.4$, so the flow field is known. The upstream and downstream areas are equal being the flow passage area, and $\mathrm{V}_{\mathrm{n}}=\mathrm{V}_{\mathrm{x}}$.

The control volume equations of continuity and momentum are shown below.

Continuity:

$$
\bar{V}_{n, d}=\bar{V}_{n, u}
$$

X-momentum:

$$
\int_{u} P d A+\int_{u} \rho V_{n} V_{x} d A=\left[P_{d}+\rho V_{n, d}^{2}\right] A
$$

Y-momentum:

$$
\int_{u} \rho V_{n} V_{y} d A=\rho \bar{V}_{n, d} \bar{V}_{y, d} A
$$

where: $\quad A=$ passage area

$d A=$ differential area

$u=$ measurement plane 


$$
\mathrm{d}=\text { mixed out plane }
$$

The mixed out flow properties are presented in the following table.

Table 4. Mixed Out Flow Properties

\begin{tabular}{|c|c|}
\hline $\bar{V}_{x, d}(\mathrm{~m} / \mathrm{s})$ & 14.4 \\
\hline $\bar{V}_{y, d}(\mathrm{~m} / \mathrm{s})$ & -29.3 \\
\hline $\bar{\beta}_{d}\left({ }^{\circ}\right)$ & 26.1 \\
\hline $\bar{C}_{p s, d}$ & -1.968 \\
\hline $\bar{C}_{p t, d}$ & 0.427 \\
\hline
\end{tabular}

The area-averaged flow angle, $\bar{\beta}_{d}=\tan ^{-1}\left[\frac{\bar{V}_{x, d}}{\bar{V}_{y, d}}\right]$, is $26.1^{\circ}$ showing the mixed out flow is essentially following the exit blade angle. The area-averaged total pressure loss coefficient defined as

$$
\bar{C}_{p t, d}=\frac{P_{t o}-P_{t, d}}{\frac{1}{2} \rho U_{0}^{2}}
$$

where

$$
P_{t, d}=P_{d}+\frac{1}{2}\left[\bar{V}_{n, d}^{2}+\bar{V}_{y, d}^{2}\right]
$$

is 0.427 . This is a considerable increase from the value of 0.375 at the measurement plane. Because the flow is uniform at the mixed out plane, $V_{n}$ is constant across the flow field, and these area average properties are equivalent to mass averaged properties. 


\subsection{GENERAL DISCUSSION}

In the earlier work of Moore and Adhye [ 16 ], it was noted that the increase in total pressure loss coefficient due to flow mixing was almost entirely explained by the dissipation of secondary kinetic energy. For this cascade with tip leakage, it is not the case. The sum of $\overline{\overline{\mathrm{C}}}_{\mathrm{pt}}$ and $\frac{\overline{\overline{\mathrm{V}^{2}+\mathrm{W}^{2}}}}{\mathrm{U}_{0}^{2}}$ at the actual measure plane is 0.391 ( $\mathrm{Ta}$ ble 3 ) which is significantly lower than the mixed out $\overline{\bar{C}}_{p t, d}$ of 0.427 . Thus, the mixing process of the tip leakage vortex seems to involve the dissipation of primary as well as secondary kinetic energy.

In the following table, a comparison of the present tip leakage cascade with Moore's older cascade is made.

Table 5. Comparison of Tip Leakage Cascade with Non-leakage Cascade

\begin{tabular}{|c|c|c|c|}
\hline & Tip Leakage Cascade & Old Cascade & Change \\
\hline$\overline{\bar{C}}_{p t} \times / c=1.4$ & 0.375 & 0.347 & 0.028 \\
\hline$\frac{\overline{v^{2}+w^{2}}}{U_{0}^{2}}, \times / c=1.4$ & 0.016 & 0.068 & -0.052 \\
\hline$\overline{\bar{C}}_{p t}+\frac{\overline{v^{2}+w^{2}}}{U_{0}^{2}}, x / c=1.4$ & 0.391 & 0.415 & -0.024 \\
\hline$\overline{\bar{C}}_{p t, d}$ & 0.427 & 0.403 & 0.024 \\
\hline
\end{tabular}

The significant differences between the two flows are the amount of secondary kinetic energy and the magnitude of the maximum loss at $x / c=1.4$, the old cascade having four times as much secondary kinetic energy while its maximum total pressure loss coefficient $\left(\overline{\bar{C}}_{p t}=1.4\right)$ was only half that of the tip leakage cascade $\left(\overline{\bar{C}}_{p t}=2.8\right)$. The 
thick inlet boundary layers of the old cascade produced large passage vortices creating a situation of comparable overall loss level at $x / c=1.4$. The mixed out losses were also similar but the process of their production appears to be different.

Qualitative estimates of the amount of secondary flow in each cascade give an insight to the differences between the two flows. In the old cascade, the thick inlet boundary layers which developed into passage vortices represented approximately $1 / 3$ of the total passage flow [ 16 ]. In the present cascade, $5.7 \%$ of the passage flow goes through the tip gap and another $1.8 \%$ is in the inlet boundary boundary layers. Thus, by this estimate, the tip leakage cascade has about $7.5 \%$ of its total passage flow producing secondary effects. The old cascade, according to this estimate, contained slightly greater than four times as much secondary flow. Therefore, the relatively small amount of secondary kinetic energy produced in the tip leakage cascade can be expected.

In addition, from the same estimates, a single passage vortex from the old cascade contains almost 3 times as much fluid as the present leakage vortex. Remembering that the secondary flows from the inlet bounary layer are very small for the present cascade and that the overall loss levels of the two cascades are approximately equal at $x / c=1.4$, a large concentration of high loss fluid within the leakage vortex is indicated.

The mixing loss of the tip leakage vortex is seen to be different from that of a passage vortex. The noted difference is apparently caused by the relatively high loss concentrated in the tip leakage fluid which does not allow the reversible exchange of primary kinetic energy for static pressure. 


\section{Chapter 7}

\section{LOSS PRODUCTION MODELS}

\subsection{ADDITIONAL LOSSES DUE TO TIP LEAKAGE}

The presence of the apparent profile loss near mid-span at $\mathrm{x} / \mathrm{c}=1.4$ discussed in section 6.5 .4 , led to the splitting up of the passage flow into two parts, the bottom half being dominated by the leakage flow and the top half containing a passage vortex. If, as suggested by Figures 43 and 45 , the profile loss does in fact exist, the secondary flows of each half do not interact, and the difference between the mass-averaged total pressure loss coefficients of the top and bottom halves is a measure of the extra loss resulting from tip leakage.

The portions of the overall mass-averaged total pressure loss coefficient produced in the top and bottom halves of the flow passage were calculated. These values and their difference are shown below. 
Bottom half: $\overline{\overline{\mathrm{C}}}_{\mathrm{pt}}=0.275$

minus

Top half: $\quad \overline{\bar{C}}_{p t}=0.101$

equals

Difference: $\quad \overline{\overline{\mathrm{C}}}_{\mathrm{pt}}=0.174$

The difference, $\overline{\bar{C}}_{p t}=0.174$, represents an additional loss due to tip leakage of $87 . \%$. That is, the present cascade would have a mass-averaged total pressure loss coefficient that is $87 . \%$ greater than that of a similar cascade having identical inlet conditions, but no tip gap.

Using the Roelke correlation, an increase in $\overline{\bar{C}}_{p t}$ due to tip leakage of $77 . \%$ was predicted as seen in Appendix A. This compares fairly well to the increase of $87 . \%$ calculated by the above method for the flow at $x / c=1.4$. If the same procedure is used with mixed out values of $\overline{\bar{C}}_{p t}$, an increase of $106 \%$ is indicated. The further increase is probably due to the noted difference in the mixing process of the tip leakage vortex.

Another estimate of the additional tip leakage loss can be made from secondary kinetic energy considerations and the flow measurements at the tip gap exit plane. By treating the flow in the bottom half of the passage as a symmetric reflection of the top half flow and superimposing the tip leakage flow upon it, the following analysis can be made.

At the tip gap exit, a mass-averaged total pressure loss coefficient of 0.071 already exists and the normal or secondary kinetic energy coefficient is 0.109 . The 
amount of the tip gap normal kinetic energy which has not been dissipated may be estimated from the difference between the portions of the total secondary kinetic energy in the bottom and top halves of the passage.

$$
\text { Bottom half: } \frac{\overline{\overline{v^{2}+w^{2}}}}{U_{0}^{2}}=0.013
$$

minus

$$
\text { Top half: } \quad \frac{\overline{\overline{v^{2}+w^{2}}}}{U_{0}^{2}}=0.002
$$

equals

$$
\text { Difference: } \quad \frac{\overline{\overline{v^{2}+w^{2}}}}{\overline{U_{0}^{2}}}=0.011
$$

The difference, $\frac{\overline{\overline{v^{2}+w^{2}}}}{U_{0}^{2}}=0.011$, is the residual tip leakage secondary kinetic energy at $x / c=1.4$. That is, of the total secondary kinetic energy generated by the tip gap ( 0.109 ), only a small amount $(0.011)$ remains at the downstream measurement plane. Therefore, by this estimate, an amount of tip gap secondary kinetic energy totaling $\frac{\overline{\bar{v}^{2}+w^{2}}}{U_{0}^{2}}=0.098$ has been dissipated by $x / c=1.4$. The extra loss due to tip leakage at $x / c=1.4$ is then,

Tip gap exit: $\quad \overline{\overline{\mathrm{C}}}_{\mathrm{pt}} \quad=0.071$

plus

$$
\text { Dissipated tip gap SKE: } \frac{\overline{\overline{v^{2}+w^{2}}}}{U_{0}^{2}}=0.098
$$

equals 
Extra tip leakage loss: $\quad \overline{\bar{C}}_{p t} \quad=0.169$

This is very close to the value of $\overline{\bar{C}}_{p t}=0.174$ reached by the previous method. It must be noted that the superposition of the two flows is not strictly valid. However, if a bottom half passage vortex, similar to that in the top half of the passage, does exist, the addition of the loss incurred within the tip gap and the amount of dissipated tip leakage secondary kinetic energy is a reasonable procedure for estimating the additional loss.

\subsection{OVERALL LOSS ACCOUNTING}

An estimate of the individual factors leading to the overall cascade loss at $x / c$ $=1.4$ was made. Integrations over the top endwall boundary layer and over the considered profile loss between $50 \%$ and $60 \%$ span were done to determine their individual contributions to the overall mass-averaged total pressure loss coefficient of 0.375 . The calculated values are listed below.

Top endwall boundary layer: $\quad \overline{\overline{\mathrm{C}}}_{\mathrm{pt}}=0.011$

Profile loss: $\quad \overline{\bar{C}}_{\mathrm{pt}}=0.157$

The profile loss may be considered to extend across the entire height of the passage.

A measure of the secondary loss of the passage vortex in the top half of the passage was made as follows. 
Overall top half loss: $\quad \overline{\bar{C}}_{p t}=0.101$

minus

Top endwall boundary layer loss: $\quad \overline{\bar{C}}_{p t}=0.011$

minus

Profile loss incurred in top half: $\quad \overline{\bar{C}}_{p t}=0.157 / 2=0.079$

equals

Top half secondary loss: $\quad \overline{\bar{C}}_{p t}=0.011$

It should be noted that this includes the $\overline{\bar{C}}_{p t}=0.002$ of the cascade top wall inlet boundary layer which provides the fluid for the passage vortex.

The overall accounting for the losses produced in this cascade are shown below.

Profile loss:

Top boundary layer:

Top passage vortex:

Tip gap loss:

Dissipated tip gap SKE:

$$
\begin{aligned}
& \overline{\bar{C}}_{p t}=0.157 \\
& \overline{\bar{C}}_{p t}=0.011 \\
& \overline{\bar{C}}_{p t}=0.011 \\
& \overline{\bar{C}}_{p t}=0.071 \\
& \overline{\bar{C}}_{p t}=0.098
\end{aligned}
$$

Assuming a bottom half passage vortex exists with identical loss and that the bottom endwall boundary has the same loss as the top endwall boundary layer, 
Bottom passage vortex: $\quad \overline{\overline{\mathrm{C}}}_{\mathrm{pt}}=0.011$

Bottom endwall boundary layer: $\quad \overline{\bar{C}}_{p t}=0.011$

Total loss at $\mathrm{x} / \mathrm{c}=1.4: \quad \overline{\overline{\mathrm{C}}}_{\mathrm{pt}}=0.370$

Again, this agrees very well with the overall measured loss at $\mathrm{x} / \mathrm{c}=1.4$ of $\overline{\overline{\mathrm{C}}}_{\mathrm{pt}}=$ 0.375. This agreement again suggests that a passage vortex does form in the bottom half of the passage.

\subsection{MIXING LOSSES}

In section 6.6, the dissipation of secondary kinetic energy was found to be an inadequate explanation of the overall mixing loss for the whole cascade. It was suggested that a difference in the mixing process of the tip leakage vortex involving the irreversable dissipation of primary kinetic energy was responsible. This dissipation of primary kinetic energy could be an explanation of why the estimate of the additional tip leakage loss based on dissipated tip gap secondary kinetic energy was slightly low.

In order to investigate this further, the top and bottom halves of the passage were treated as entirely different flows. The losses of each half were mass-averaged and then mixed out as if each half existed separately. The results of this analysis are presented in Table 6. 
Table 6. Comparison of the Top and Bottom Halves of the Flow Passage

\begin{tabular}{|c|c|c|}
\hline & Top Half Passage & Bottom Half Passage \\
\hline$\overline{\bar{C}}_{p t}, \times / c=1.4$ & 0.199 & 0.555 \\
\hline$\frac{\overline{v^{2}+w^{2}}}{U_{0}^{2}}, \times / c=1.4$ & 0.005 & 0.027 \\
\hline$\overline{\bar{C}}_{p t}+\frac{\overline{v^{2}+w^{2}}}{U_{0}^{2}}, \times / c=1.4$ & 0.204 & 0.582 \\
\hline$\overline{\bar{C}}_{p t, d}$ & 0.206 & 0.643 \\
\hline
\end{tabular}

In the case of the top half passage, with only a passage vortex, the dissipation of secondary kinetic energy is seen to account for the increased loss due to mixing, which agrees with the finding of Moore and Adhye [ 16 ]. However, in the bottom half passage, the increase in loss at the mixed out plane is significantly larger than the secondary kinetic energy at $x / c=1.4$. This further exemplifies the difference in mixing process of a tip leakage vortex when compared to a passage vortex. It also suggests that a dissipated primary kinetic energy term should be added to the overall cascade loss accounting in section 7.2 


\section{Chapter 8}

\section{CONCLUSIONS}

An experimental study of incompressible flow through a large scale turbine cascade with a tip gap measuring $2.1 \%$ of the blade height has been performed. The Reynolds number based on axial chord and exit velocity was $4.5 \times 10^{5}$. The flow exiting the tip gap was documented and its effects on the flow at 1.4 axial chords downstream of the blade leading edges were measured.

\subsection{TIP LEAKAGE MEASUREMENTS}

The flow measurements at the tip gap exit plane and the flow visualizations revealed the following information about the flow.

1. $5.7 \%$ of the total passage flow passes through the tip gap. 
2. The tip leakage flow is driven by the pressure difference across the tip gap and is strongest near mid-axial chord where the measured exit plane static pressure is lowest.

3. Measurements of the flow direction at the tip gap exit plane showed it not to be uniformly perpendicular to the blade mean camber line as previously thought, but to depend on the exit plane static pressure.

4. A loss occurs within the tip gap developing a mass-averaged total pressure loss coefficient, $\overline{\overline{\mathrm{C}}}_{\mathrm{pt}}=0.071$, by the tip gap exit plane. This loss is believed to be caused primarily by flow mixing following the vena-contracta and augmented by viscous shear and the possible effects of the observed laminar separation and turbulent reattachment of the endwall boundary layer within the tip gap.

5. The tip leakage flow produces a significant amount of secondary kinetic energy, $10.9 \%$ of the inlet passage kinetic energy. This secondary kinetic energy adds to the tip leakage loss in nearly a one for one correspondence as it dissipates downstream of the blade row.

\subsection{DOWNSTREAM MEASUREMENTS}

The measurements at $x / c=1.4$ showed the flow field to be dominated by three distinct loss cores, the most prominant of which was the tip leakage vortex. The conclusions drawn from the downstream measurements are as follows.

1. The tip leakage vortex covers about $1 / 5$ of the flow passage and contains a maximum total pressure loss coefficient of 2.8 . 
2. A distinct passage vortex exists in the top half of the passage having a maximum total pressure loss coefficient of 1.1 which is significantly lower than that of the leakage vortex.

3. A loss core exists in the bottom half of the passage and is believed to be a second passage vortex. Its lack of a definite core of solid body rotation as seen in secondary velocity vectors of the top passage vortex is probably due to its interaction with the leakage vortex.

4. The secondary flows produced in this cascade have little effect on static pressure which is seen to be fairly uniform at the measurement plane.

5. The mass-averaged total pressure loss coefficient at $x / c=1.4$ is 0.375 indicating a relatively high level of loss due to the tip leakage.

\subsection{FLOW MIXING}

A constant area mixing analysis of the measured flow at $x / c=1.4$ to a downstream plane where the flow was assumed to be uniform led to some important observations.

1. The flow as a whole had a mixed out mass-averaged total pressure loss coefficient of 0.427 which is significantly larger than that expected from the dissipation of secondary kinetic energy.

2. A difference in the mixing process of the tip leakage vortex is believed to prevent the reversible exchange of primary kinetic energy for static pressure as seen in the mixing of a passage vortex. 


\subsection{LOSS PRODUCTION MODELS}

Several loss production models were developed which provide the following information.

1. The tip leakage of this cascade produces $87 . \%$ more loss than would a similar cascade without tip leakage.

2. The sum of the internal tip gap losses and the dissipated tip leakage secondary kinetic energy gives a good estimate of the additional loss due to tip leakage at a particular flow field location.

3. The overall cascade loss at $x / c=1.4$ can be considered as twice the loss in the top half of the passage plus the additional loss due to tip leakage. In other words, the flow in the bottom half of the passage can be considered a reflection of the top half flow with the leakage loss superimposed upon it.

If a method of predicting the magnitude of loss which occurs within the tip gap and the amount secondary kinetic energy created by the leakage was developed, a very powerful tool for estimating tip leakage losses would be gained. 


\section{REFERENCES}

1. Moore, J. and Tilton, J. S. "Tip Leakage Flow in a Linear Turbine Cascade," Paper 87-GT-222, September 1987.

2. Kofskey, M. G., "Experimental Investigation of Three Tip-Clearance Configurations Over a Range of Tip Clearance Using a Single-Stage Turbine of High Hub to Tip-Radius Ratio," NASA TM X-472, 1961.

3. Kofskey, M.G., and Nusbaum, W. J., "Performance Evaluation of a Two-Stage Axial-Flow Turbine for Two Values of Tip Clearance," NASA TN D-4388, 1968.

4. Holeski, D. E., and Futral, S. M., "Effect of Rotor Tip Clearance on the Performance of a 5-Inch Single-Stage Axial-Flow Turbine," NASA TM X-1757, 1969

5. Haas, J. E., and Boyle, R. J., "Analytical and Experimental Investigation of Stator Endwall Contouring in a Small Axial-Flow Turbine," NASA TP 2309, 1984.

6. Bindon, J. P., "Pressure Distributions in the Tip Clearance Region of an Unshrouded Axial Turbine as Affecting the Problem of Tip Burnout," ASME Paper 87-GT-230, June 1987.

7. Bindon, J. P., "Pressure and Flow Field Measurements of Axial Turbine Tip Clearance Flow in a Linear Cascade," Report No. CUED/A-Turbo TR123, Whittle Laboratory, Cambridge University, August 1986.

8. Roelke, R. J., "Turbine Design and Application," Glassman, A. J., Ed., NASA SP-290, pp. 125-131, 1973.

9. Abiants, V. Kh., Theory of Aircraft Gas Turbines, Oborongiz, Moscow, 1953.

10. Hennecke, D. K., "Active and Passive Tip Clearance Control," von Karman Institute for Fluid Dynamics, Lecture Series 1985-05 on Tip Clearance Effects in Axial Turbomachines, April 1985. 
11. Booth,T. C., "Tip Clearance Effects in Axial Turbomachines," von Karman Institute for Fluid Dynamics, Lecture Series 1985-05 on Tip Clearance Effects in Axial Turbomachines, April 1985.

12. Hourmouziadis, J., and Albrecht, G., "An Integrated Aero/Mechanical Performance Approach to High Technology Turbine Design," AGARD Symposium on Advanced Technology for Aero Gas Turbine Components, Paris, France, May 1987.

13. Bindon, J. P., "Visualization of Axial Turbine Tip Clearance Using a Linear Cascade," Report No. CUED/A-Turbo TR122, Whittle Laboratory, Cambridge University, July 1986.

14. Klein, A., "Investigation of the Effect of the Entry Boundary Layer on the Secondary Flows in the Blading of Axial-Flow Turbines," Forshung Ing.-Wes., 32, 6 pp.175-187, 1966, also BHRA Translation T 1004, April 1969.

15. Sjolander, S. A., and Amrud, K. K., "Effects of Tip Clearance on Blade Loading In a Planar Cascade of Turbine Blades," ASME Paper 86-GT-245, June 1986.

16. Moore, J., and Adhye, R., "Secondary Flows and Losses Downstream of a Turbine Cascade," Trans. ASME, Journal of Engineering for Gas Turbines and Power, Vol. 107, No. 4, pp. 961-968, Oct. 1985.

17. Langston, L. S., Nice, M. L., and Hooper, R. M., "Three-Dimensional Flow Within a Turbine Cascade Passage," Trans. ASME, Journal of Engineering for Power, Vol. 99, pp. 21-28, Jan. 1977.

18. Ransmayr, A., "The Influence of Leading Edge Shape on the Overall Losses in a Turbine Cascade," M.S. Thesis, Virginia Polytechnic and State University, Blacksburg, Virginia, August 1981.

19. Adhye, R. Y., "Secondary Flows and Losses Downstream of a Turbine Cascade," M.S. Thesis, Virginia Polytechnic and State University, Blacksburg, Virginia, April 1984.

20. Tilton, J. S., "Tip Leakage Flow in a Linear Turbine Cascade," M.S. Thesis, Virginia Polytechnic and State University, Blacksburg, Virginia, August 1986.

21. Moore, J., "Flow Trajectories, Mixing and Entropy Fluxes in a Turbine Cascade," AGARD Conference Proceedings No. CP-351 on Viscous Effects in Turbomachines, Copenhagen, Denmark, June 1983.

22. Dixon, S. L., Fluid Mechanics, Thermodynamics of Turbomachinery, Pergamon Press, 1966. 


\section{Appendix A}

\section{TIP LEAKAGE LOSS ESTIMATE}

An estimate of the extra loss due to tip leakage for the present turbine rotor cascade was made using Roelke's correlation of turbine efficiency [ 8 ]. A turbine configuration including the present cascade as the turbine blade row was postulated. This is shown in Figure 50.

The nozzle exit angle $\theta_{2}$ was taken to be $70^{\circ}$.

The loss without tip leakage was first estimated using Soderberg's correlation [ 22 ]. Following Dixon [ 22 ], the total-to-total turbine efficiency is given approximately by

$$
\eta_{\mathrm{tt}}=\left[1+\frac{\zeta_{\mathrm{R}} \frac{\mathrm{W}_{3}^{2}}{2}+\zeta_{N} \frac{\mathrm{V}_{2}^{2}}{2}}{U\left(\mathrm{~V}_{\theta 2}-\mathrm{V}_{\theta 3}\right)}\right]^{-1}
$$

where: $\quad \zeta_{R}=$ rotor loss coefficient 


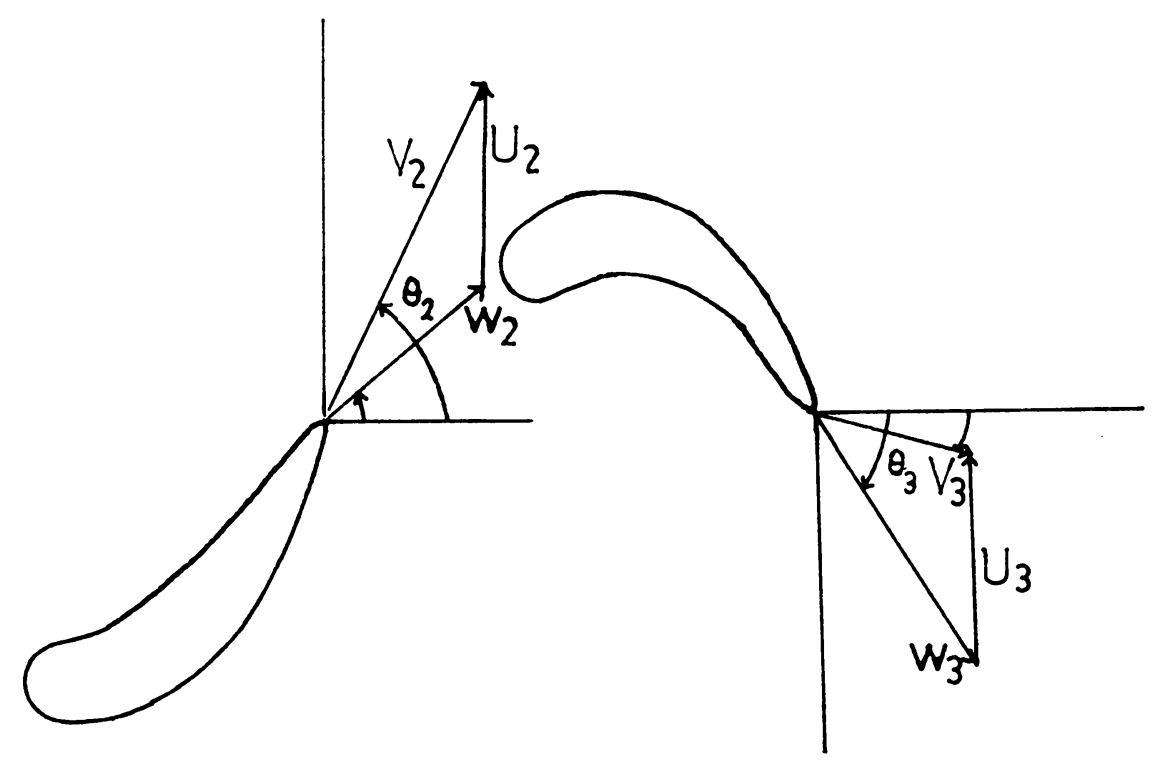

Figure 50. Turbine Configuration and Geometry 


$$
\begin{aligned}
& \zeta_{N}=\text { nozzle loss coefficient } \\
& V=\text { absolute velocity } \\
& W=\text { relative velocity } \\
& U=\text { blade speed } \\
& 2=\text { nozzle exit, rotor inlet } \\
& 3=\text { rotor exit } \\
& \theta \text { circumferential component }
\end{aligned}
$$

The intermediate steps are not included but a total-to-total efficiency, $\eta_{\pi}=$ $90 \%$, was calculated corresponding to $\zeta_{R}=0.130$.

Assuming that a change in $\eta_{\pi}$ with tip leakage is due only to a change in $\zeta_{R}$,

$$
\frac{\eta^{\prime}{ }_{\mathrm{tt}}}{\eta_{\mathrm{tt}}}=\frac{u\left(\mathrm{~V}_{\theta 2}-\mathrm{V}_{\theta 3}\right)+\zeta_{\mathrm{R}} \frac{\mathrm{W}_{3}^{2}}{2}+\zeta_{N} \frac{\mathrm{V}_{2}^{2}}{2}}{U\left(\mathrm{~V}_{\theta 2}-\mathrm{V}_{\theta 3}\right)+\zeta_{R}^{\prime} \frac{\mathrm{W}_{3}^{2}}{2}+\zeta_{N} \frac{\mathrm{V}_{2}^{2}}{2}}
$$

where' indicates corrected for tip leakage.

From Roelke's correlation ( Figure 1), $\frac{\eta_{\pi}^{\prime}}{\eta_{\pi}}=0.957$ for the present rotor with a tip clearance of $2.1 \%$ of the passage height and a reaction of 0.27 .

Again, the intermediate steps are not shown, but a rotor loss coefficient corrected for tip leakage, $\zeta_{R}^{\prime}=0.232$, was calculated.

Noting:

$$
C_{p t}=\frac{P_{02}-P_{03}}{\rho \frac{W_{2}^{2}}{2}},
$$


and

$$
\begin{aligned}
& \frac{1}{2} W_{3}^{2} \zeta_{R} \simeq \frac{P_{02}-P_{03}}{\rho}, \\
& C_{p t} \simeq \zeta_{R}\left[\frac{W_{3}}{W_{2}}\right]^{2}
\end{aligned}
$$

giving $\quad C_{p t} \simeq 0.335$

and $\quad C_{p t}^{\prime} \simeq 0.597$

Accounting for the inlet boundary layer loss of the cascade, $\overline{\bar{C}}_{p t}=0.004$,

$$
\begin{aligned}
C_{p t} & =0.339 \\
\text { and } \quad C_{p t}^{\prime} & =0.601
\end{aligned}
$$

This indicates a $77 . \%$ increase in $C_{p t}$ due to tip leakage. 
The vita has been removed from the scanned document 